\title{
Circumstellar shells and mass loss rates: Clues to the evolution of S stars ${ }^{\star}$
}

\author{
A. Jorissen ${ }^{1,2, \star \star}$ and G.R. Knapp ${ }^{2}$ \\ 1 Institut d'Astronomie et d'Astrophysique, Université Libre de Bruxelles, CP. 226, boulevard du Triomphe, B-1050 Bruxelles, \\ Belgium \\ 2 Department of Astrophysical Sciences, Princeton University, Princeton, NJ 08544, U.S.A.
}

Received July 25; accepted October 13, 1997

\begin{abstract}
It is the purpose of this paper to rediscuss the circumstellar properties of S stars and to put these properties in perspective with our current understanding of the evolutionary status of S stars, in particular the intrinsic/extrinsic dichotomy. This dichotomy states that only Tc-rich ("intrinsic") S stars are genuine thermally-pulsing asymptotic giant branch stars, possibly involved in the MS-C evolutionary sequence. Tc-poor S stars are referred to as "extrinsic" S stars, because they are the cooler analogs of barium stars, and like them, owe their chemical peculiarities to mass transfer across their binary system.

Accordingly, an extensive data set probing the circumstellar environment of S stars (IRAS flux densities, maser emission, CO rotational lines) has been collected and critically evaluated. This data set combines new observations (9 stars have been observed in the CO $J=2-1$ line and 3 in the CO $J=3-2$ line, with four new detections) with existing material (all $\mathrm{CO}$ and maser observations of $\mathrm{S}$ stars published in the literature). The IRAS flux densities of $\mathrm{S}$ stars have been re-evaluated by co-adding the individual scans, in order to better handle the intrinsic variability of these stars in the IRAS bands, and possible contamination by Galactic cirrus.
\end{abstract}

In the $(K-[12],[25]-[60])$ color-color diagram, S stars segregate into five distinct regions according to their Tc content and $\mathrm{ZrO} / \mathrm{TiO}, \mathrm{C} / \mathrm{O}$ and IR spectral indices. Stars with photospheric colors (populating "Region A") may be identified with extrinsic S stars. For the other regions characterized by different excess levels in the 12, 25 and $60 \mu \mathrm{m}$ bands, several diagnostics (like the IRAS spectral class, maser emission, and shape of $\mathrm{CO}$ rotational lines) have been collected to infer the physical properties of the dust shell. A simple radiative-transfer code has also been used to infer the chemical nature (carbonaceous or sili-

Send offprint requests to: A. Jorissen (at the address in Belgium).

* Based on observations carried out at the Caltech Submillimeter Observatory (Mauna Kea, Hawaii).

$\star \star$ Research Associate, F.N.R.S., Belgium. cate) of the dust grains from the observed IR colors. S stars with large $K-[12]$ excesses and moderate [25] [60] excesses (populating Regions B and C) exhibit the signatures of oxygen-rich shells $(9.7 \mu \mathrm{m}$ silicate emission and $\mathrm{SiO}$ maser emission). The situation is less clear for $\mathrm{S}$ stars with small $K-[12]$ and moderate [25] - [60] indices (populating Regions D and E). Their IR colors are consistent with carbonaceous grains (as is their featureless IRAS spectrum, and absence of silicate or $\mathrm{SiO}$ maser emission), but these features may equally well be explained by a detached shell. For many of these stars with a large $60 \mu \mathrm{m}$ excess, the shell is indeed resolved by the IRAS beam at $60 \mu \mathrm{m}$. The prototypical SC star FU Mon is among these. Since SC stars are believed to be in a very short-lived evolutionary phase where $\mathrm{C} / \mathrm{O}=1$ within $1 \%$, FU Mon may be a good candidate for the "interrupted mass-loss" scenario advocated by Willems \& de Jong (1988). The CO line profile of FU Mon is also peculiar in being quite narrow $\left(V_{\mathrm{e}}=2.8 \mathrm{~km} \mathrm{~s}^{-1}\right)$, suggesting that the mass loss has just resumed in this star.

Mass loss rates or upper limits have been derived for all $\mathrm{S}$ stars observed in the $\mathrm{CO}$ rotational lines, and range from $<210^{-8} M_{\odot} \mathrm{y}^{-1}$ for extrinsic $\mathrm{S}$ stars to $110^{-5} M_{\odot} \mathrm{y}^{-1}$ (the Mira S star W Aql). These mass-loss rates correlate well with the $K-[12]$ color index, which probes the dust loss rate, provided that $\dot{M} \gtrsim 10^{-8} M_{\odot} \mathrm{y}^{-1}$. Small massloss rates are found for extrinsic $\mathrm{S}$ stars, consistent with their not being so evolved (RGB or Early-AGB) as the Tc-rich S stars. This result does not support the claim often made in relation with symbiotic stars that binarity strongly enhances the mass-loss rate.

Key words: stars: mass-loss — stars: AGB — stars: latetype - infrared: stars — radio lines: stars 


\section{Introduction}

The S stars are late-type giants whose spectra resemble those of $\mathrm{M}$ giants, with the addition of distinctive molecular bands of $\mathrm{ZrO}$ (Merrill 1922). The presence of $\mathrm{ZrO}$ bands is often considered as a direct consequence of molecular equilibrium in the special circumstances where the atmospheric $\mathrm{C} / \mathrm{O}$ ratio is within $10 \%$ of unity (e.g. Scalo \& Ross 1976). However, Piccirillo (1980) has shown that the above statement is only valid for stars with $T<3000 \mathrm{~K}$. At higher temperatures, an enhanced $\mathrm{Zr}$ abundance, rather than a $\mathrm{C} / \mathrm{O}$ ratio close to unity, is the dominant factor in the development of strong $\mathrm{ZrO}$ bands. Detailed abundance analyses (Smith \& Lambert 1990) have shown that the envelopes of S stars are enriched in heavy elements like $\mathrm{Zr}$, and so bear the signature of the $s$-process of nucleosynthesis (Käppeler et al. 1989). Although larger than in $\mathrm{M}$ giants, the $\mathrm{C} / \mathrm{O}$ ratio of $\mathrm{S}$ stars is not necessarily close to unity (Smith \& Lambert 1990), except in the so-called SC stars (Dominy et al. 1986).

When S stars were still believed to be objects with $\mathrm{C} / \mathrm{O}$ close to unity, they were naturally considered as transition objects between $\mathrm{M}$ giants $(\mathrm{C} / \mathrm{O}<1)$ and carbon stars $(\mathrm{C} / \mathrm{O}>1)$ on the asymptotic giant branch $(\mathrm{AGB})$ (Iben \& Renzini 1983). Support to this scenario is provided by observations of $\mathrm{S}$ stars on the upper AGB of globular clusters in the Magellanic Clouds (Bessell et al. 1983; Lloyd Evans 1983-1985). In this evolutionary phase, low- and intermediate-mass stars are characterized by a double $(\mathrm{H}, \mathrm{He})$ burning-shell structure which is thermally unstable. The thermal instabilities ("thermal pulses") developing in the He-burning shell are the site of a rich nucleosynthesis (Frost \& Lattanzio 1995), probably including the $s$-process, although its detailed mode of operation remains poorly understood (e.g. Sackmann \& Boothroyd 1991; Herwig et al. 1997). In the receding phase of the thermal instability, the convective outer envelope may plunge ("third dredge-up") into the intershell zone containing the He-burning ashes, and bring fresh carbon and $s$-process elements to the surface.

However, several observations have challenged this traditional M-S-C evolution sequence. The first set of observations relates to Tc, an element with no stable isotopes, discovered in the spectra of some S stars by Merrill (1952). If the $s$-process indeed occurred during recent thermal pulses in $\mathrm{S}$ stars, Tc should be observed at the surface along with the other $s$-process elements (Mathews et al. 1986). Little et al. (1987) found however that only longperiod Mira or semiregular S stars (i.e., intrinsically bright $\mathrm{S}$ stars) exhibit Tc lines. Second, the broad range of IRAS colors exhibited by S stars (e.g., Jorissen et al. 1993) is difficult to reconcile with the idea that they represent a brief transition phase as the star evolves from an oxygenrich $\mathrm{M}$ giant with $\mathrm{C} / \mathrm{O}<1$ into a $\mathrm{C}$ star with $\mathrm{C} / \mathrm{O}>1$. $\mathrm{M}$ and $\mathrm{C}$ stars occupy well-defined regions in the IRAS colorcolor diagram, and it is not clear how $\mathrm{S}$ stars fit into the (much debated) evolutionary sequence joining $\mathrm{M}$ stars to C stars in that diagram (Willems \& de Jong 1986, 1988; Chan \& Kwok 1988; Zuckerman \& Maddalena 1989; de Jong 1989).

These problems received a new impetus with the discovery that the barium stars, a family of peculiar red giant (PRG) stars of spectral type $\mathrm{G}$ and $\mathrm{K}$, are all members of binary systems (McClure et al. 1980; McClure 1983). Iben \& Renzini (1983) were the first to propose that Tc-poor S stars could perhaps be the cooler analogs of the barium stars. Long-term radial-velocity monitoring confirmed this suggestion, and it is now clear that Tcpoor S stars are binary stars (Smith \& Lambert 1988; Brown et al. 1990; Jorissen \& Mayor 1992; Jorissen et al. 1993; Johnson et al. 1993) with orbital elements identical to those of barium stars (Jorissen et al. 1998). Tc-poor S stars are now referred to as "extrinsic S stars", because, like barium stars, they owe their chemical peculiarities to mass transfer across the binary system. On the contrary, Tc-rich, "intrinsic S stars" are genuine thermally-pulsing (TP) stars on the TP-AGB. Since the C/O ratio of extrinsic $\mathrm{S}$ stars depends on the details of the mass accretion process, it is not necessarily close to unity, but as discussed above, neither abundance analyses nor predictions of molecular chemical equilibrium really require $\mathrm{C} / \mathrm{O}$ to be close to unity in $\mathrm{S}$ stars.

In Paper I (Jorissen et al. 1993; see also Groenewegen 1993), it was shown that the correlation Tc-poor/binary found for $\mathrm{S}$ stars could be extended to their IRAS colors, since another distinctive property of binary, Tc-poor $\mathrm{S}$ stars is the absence of IR excesses. Their IRAS colors simply reflect the photospheric colors, contrary to Tc-rich $\mathrm{S}$ stars which usually exhibit IR excesses. These IR excesses are caused by circumstellar dust, and are indicative of substantial mass loss, thus suggesting that Tc-rich S stars are more massive and/or more evolved than Tc-poor S stars.

The possibility offered by the circumstellar properties of S stars to probe their evolutionary status has been used in several recent studies (Jura 1988; Chen \& Kwok 1993; Bieging \& Latter 1994; Sahai \& Liechti 1995). However, these studies still rely on hypotheses not fully consistent with the dichotomy recently found among S stars, as discussed above, and their conclusions may therefore be somewhat biased. For example, it makes no sense to test the AGB evolutionary sequence $\mathrm{M}-\mathrm{S}-\mathrm{C}$ using a sample of $\mathrm{S}$ stars not properly cleaned from its extrinsic content. In addition, models inferring the chemical nature (carbonaceous or silicate) of the dust grains from models assuming that $\mathrm{C} / \mathrm{O}$ is close to unity in the photosphere do not sample the whole parameter space occupied by these stars. A similarly incorrect corollary consists of concluding that dust production, and thus mass loss, is not very efficient in $\mathrm{S}$ stars because dust-seed molecules no longer form in the absence of any free $\mathrm{C}$ or $\mathrm{O}$ atoms, since these are all tied up in $\mathrm{CO}$ when $\mathrm{C} / \mathrm{O} \sim 1$. 
It is the purpose of this paper to rediscuss the circumstellar properties of S stars and to put these properties in perspective with our current understanding of the evolutionary status of S stars, in particular the intrinsic/extrinsic dichotomy. An extensive data set probing the circumstellar environment of S stars (IRAS colors, maser emission, CO rotational lines) has therefore been collected and critically evaluated. This data set combines new material with existing results collected from the literature.

The IRAS colors of S stars provide a first way to probe their circumstellar environment, and more specifically, to evaluate the amount and nature of the dust surrounding these objects. A re-evaluation of the flux densities listed in the IRAS Point Source Catalogue (IRAS Science Team 1988; PSC) was necessary to circumvent the problems of interpretation related to the intrinsic IR variability of these sources and to their possible contamination by Galactic cirrus emission. These effects are not always properly handled in the PSC. This re-evaluation was performed by co-adding the raw scans (Sect. 2.2). The clean flux densities were then used to define five regions in the $(K-[12],[25]-[60])$ color-color diagram (Sect. 2.3) which contain stars of similar extrinsic or intrinsic nature and of similar $\mathrm{ZrO} / \mathrm{TiO}, \mathrm{C} / \mathrm{O}$ and IR spectral indices. Several $\mathrm{S}$ stars with shells resolved at 60 (and sometimes 100) $\mu \mathrm{m}$ have been found by comparing the source profile with the IRAS point source response function (Sect. 2.4). Resolved shells at $60 \mu \mathrm{m}$ appear to be correlated with large $60 \mu \mathrm{m}$ excesses, suggesting that these shells have detached from their parent star. A simple model of the dust shell has been used to predict its IR colors and to infer the chemical nature of the dust grains (Sect. 4), using constraints provided by the detection or non-detection of maser emission (Sect. 3). Finally, mass loss rates have been derived in an homogeneous way (Sect. 5.3) from the intensities of the $\mathrm{CO}$ millimeter-wave lines, derived from new observations with the Caltech Submillimeter Observatory (Sect. 5.1) or collected from the literature (Sect. 5.2). The mass loss rates, wind expansion velocities, IR colors and extrinsic/intrinsic nature are then discussed together in Sect. 6.

\section{Infrared colors of S stars}

\subsection{The sample}

The sample of $\mathrm{S}$ stars considered in this paper was selected from the list of Chen et al. (1995), which provides associations between IRAS sources from the PSC and S stars from the General Catalogue of Galactic S Stars (GCGSS, Stephenson 1984) and from an additional later list (Stephenson 1990). Among these, only stars having flux densities of good quality (i.e. with a quality flag of 3 in the PSC) in the 12,25 and $60 \mu \mathrm{m}$ bands have been retained.
Several stars that have probably been misclassified as $\mathrm{S}$ stars were removed from the final sample. For example, stars from the original Westerlund \& Olander (1978) sample were later recognized by Lloyd Evans \& Catchpole (1989) as actually being heavily-reddened M giants or supergiants. A few other cases of $\mathrm{M}$ giants or supergiants misclassified as S stars were identified by Keenan \& McNeil (1989; HR 3296 = GCGSS 500) and Winfrey et al. (1994; GCGSS 1314 and star 41 in Stephenson 1990). A detailed heavy-element abundance analysis (Lambert et al. 1995) has shown that the stars DE Leo (HR 4088) and HR 7442, although often considered as S stars, have normal abundances. According to Meadows et al. (1987), GCGSS 886 (IRAS 15194 - 5115) is now classified as a carbon star and thus has not been retained in the final sample of $\mathrm{S}$ stars. Our final sample may still be somewhat contaminated by $\mathrm{M}$ supergiants misclassified as $\mathrm{S}$ stars and by a few carbon stars. The star T Cet for example was classified as M5-6Se in the original paper by Keenan (1954) defining the $\mathrm{S}$ class, but it was reclassified as M5/M6Ib/II in the Michigan Spectral Survey (Houk \& Cowley 1975). Since at the low plate dispersions used in classification work, these two types of spectra look similar (e.g. Lloyd Evans \& Catchpole 1989), $\mathrm{T}$ Cet has been kept in our final list until higher resolution spectra resolve these conflicting classifications. The same holds true for TV Dra and OP Her (see Table 2 of GCGSS). Note, however, that SC stars like RZ Peg, FU Mon and GP Ori are retained in the sample, since they may provide important clues to the evolutionary status of $\mathrm{S}$ stars as a whole. Similarly interesting are the two CS stars TT Cen and BH Cru that are known to exhibit $\mathrm{ZrO}$ bands at some times and $\mathrm{C}_{2}$ bands at others (Stephenson 1973; Lloyd Evans 1985).

\subsection{The IRAS flux densities}

The final sample consists of $124 \mathrm{~S}$ stars having flux densities at 12,25 and $60 \mu \mathrm{m}$ flagged as being of good quality in the PSC. These stars are listed in Table 1. However, PSC flux densities suffer from several shortcomings that make them inadequate for the present study. First, the $60 \mu \mathrm{m}$ flux density may in some cases be seriously contaminated by Galactic cirrus emission, as shown by Ivezić \& Elitzur (1995). Second, the PSC flux densities are not appropriate in the case of slightly extended or variable sources, as several S stars appear to be. Moreover, hysteresis of the detectors hampers the search for extended sources and should be properly identified. Finally, the detectors may saturate on very bright sources, those with flux densities in excess of $1000 \mathrm{Jy}$ (like $\chi$ Cyg, see Appendix A).

In order to correctly handle these effects, the raw IRAS data for all $\mathrm{S}$ stars in our sample were reprocessed through the ADDSCAN procedure provided by 
the Infrared Processing and Analysis Center (IPAC $\left.{ }^{1}\right)$. This procedure has been used to co-add all scans passing within 1.7 of the target position. Before being co-added, the raw data are first interpolated using cubic splines and resampled at 10 data points per arcminute in all bands. A baseline is then defined for each individual scan by fitting a parabola to the data in a window extending from $r_{\text {in }}$ to $r_{\text {out }}$ from the target $\left(r_{\text {in }}= \pm 2^{\prime}, \pm 2^{\prime}, \pm 2 \cdot 5, \pm 4^{\prime}\right.$ and $r_{\text {out }}= \pm 7 ! 5, \pm 7 ! 5, \pm 10^{\prime}, \pm 13.5$ in the $12,25,60$ and $100 \mu \mathrm{m}$ bands, respectively). The rms residual $\sigma_{i}$ of the data around the baseline fit is an indication of the background noise in a given scan $i$ (note that possible nearby sources with a peak flux density exceeding $2.5 \sigma_{i}$ are automatically removed and do not enter the final residual calculation). The different scans are then co-added with weighting factors equal to $1 / \sigma_{i}^{2}$. The noise $\sigma$ in the coadded scan, computed in a similar way as for the individual scans, is smaller than that in any individual scan, and the reduction may be substantial when scans with very different orientations, sampling different regions of the sky around the target, are available, since structure in the Galactic cirrus emission contributes to the noise. Finally, a point-source template with adjustable width is fitted to the co-added scan in the target window $\pm r_{\text {in }}$. Three different estimates of the flux densities in each band are then computed: the peak flux density $F_{\mathrm{p}}$, the template flux density $F_{\mathrm{t}}$ and the "zero-crossing" flux density $F_{\mathrm{z}}$. The template flux density $F_{\mathrm{t}}$ is derived from the template fit to the data, and generally agrees with $F_{\mathrm{p}}$, unless the source is extended or hysteresis effects are important. For extended sources, the "zero-crossing" flux density $F_{z}$ must be preferred. It corresponds to the integrated flux density between the "zero crossings", which are defined as the first locations, moving outwards from the peak location, where the source profile intersects the baseline. In Sect. 2.4, a criterion based on the comparison of $F_{\mathrm{z}}$ and $F_{\mathrm{p}}$ has been designed to identify sources with possibly resolved shells. If that criterion is met, the $F_{z}$ flux density (identified by a "+" in Table 1) has been adopted instead of $F_{\mathrm{t}}$. In the case of bright sources, detector hysteresis results in a trail extending along the scan direction, thus disturbing the template fit. In that case, $F_{\mathrm{p}}$ has been adopted. The template fit to sources embedded in strong Galactic cirrus emission generally resulted in an abnormally narrow profile, because the adopted baseline is then too high with respect to the base of the source signal. These contaminated sources were thus readily identified by their narrow template fit, and were rejected. Finally, individual scans clearly contaminated by nearby sources have been eliminated.

The adopted IRAS flux densities are listed in Table 1 in Cols. 5-8. The $2.2 \mu \mathrm{m}$ flux density is listed in Col. 3. The calibration of Beckwith et al. (1976; 620 Jy corre-

\footnotetext{
$1 \quad$ IPAC is operated by the Jet Propulsion Laboratory and California Institute of Technology for NASA.
}

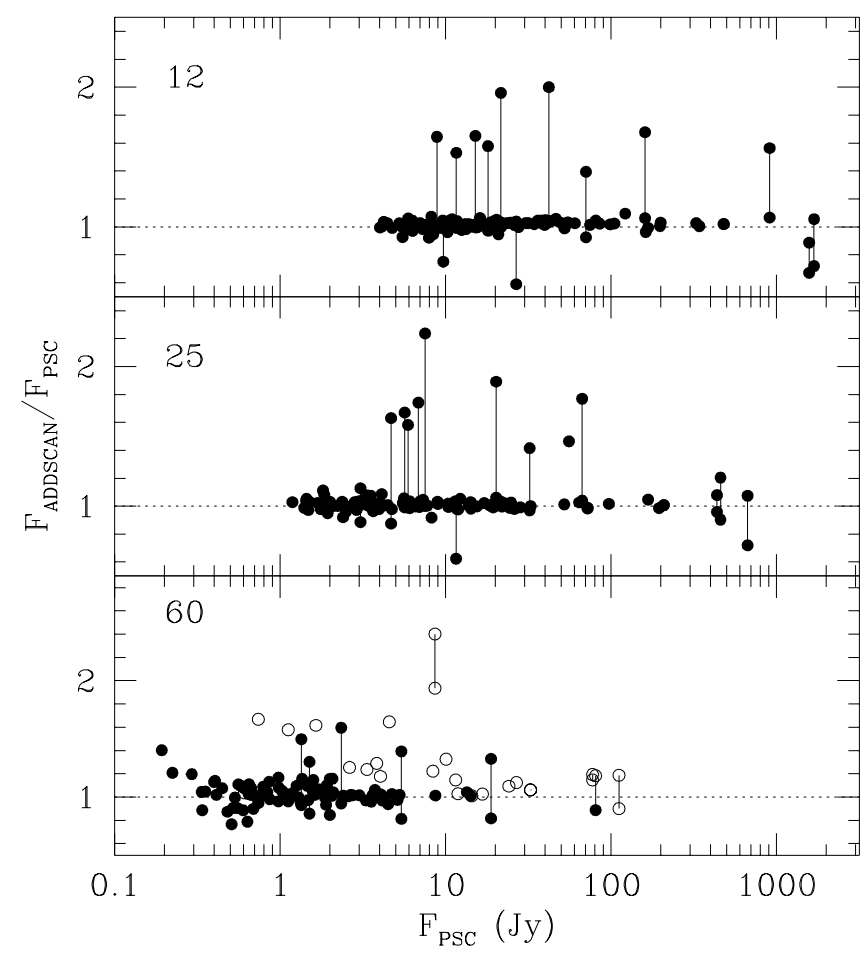

Fig. 1. Comparison between the PSC flux densities and those derived from the ADDSCAN procedure (see text) in the 12, 25 and $60 \mu \mathrm{m}$ bands. Flux densities of variable sources at two different epochs are connected by a vertical line. At $60 \mu \mathrm{m}$, open circles correspond to extended sources (see Sect. 2.4)

sponds to $K=0$ ) has been used to derive the $2.2 \mu \mathrm{m}$ flux density from the $K$ magnitude provided by different authors, as listed in Col. 4: 1: Catchpole et al. (1979); 2: Neugebauer \& Leighton (1969; Two-Micron Sky Survey); 3: Mendoza \& Johnson (1965); 4: Price (1968); 5: Chen et al. (1988); 6: Noguchi et al. (1991); 7: Guglielmo et al. (1993); 8: Epchtein et al. (1990); 9: Epchtein et al. (1987); 10: Lloyd Evans \& Catchpole (1989).

The Tc content (from Paper I, and Lambert et al. 1995) is listed in Col. 9. The Cols. labelled "LRS" and "VC" provide the classification of the IRAS low-resolution spectrum, according to the IRAS Low Resolution Spectrometer Catalogue (Olnon et al. 1986) or to the Volk \& Cohen (1989) schemes, respectively. The optical spectral type is from the GCGSS. The variability type, the period and the range of variation of the magnitude (lowest minimum - highest maximum) are from the General Catalogue of Variable Stars (Kholopov et al. 1985; GCVS). The variable name and/or the HD/HR designations, when available, are given in the last column. If maser emission has been detected (see Sect. 3 and Table 3 ), the maser type ( $\mathrm{SiO}$ or $\mathrm{OH})$ is given between parentheses after the stellar designation.

Several sources observed by IRAS a few months apart turn out to be strongly variable in the IRAS bands. 
For these, the ADDSCAN procedure has been run separately on the two groups of data, and the flux densities are listed on two separate lines in Table 1. The corresponding approximate Julian dates have been derived from the "Satellite Operation Plan" number attached to the scans and from the mission chronology provided in Table III.C.1 of the IRAS Explanatory Supplement (1988).

The flux densities derived from the ADDSCAN procedure generally agree with the PSC flux densities within $20 \%$, as shown in Fig. 1. In all bands, the scatter is larger at low flux densities. Several stars show much larger deviations because of intrinsic variability. For these, it turns out that the PSC flux densities correspond to one epoch, whereas the flux densities at the other epoch may differ by as much as a factor of 2. An example of a strongly variable source, $\chi \mathrm{Cyg}$, is discussed in Appendix A. In the $60 \mu \mathrm{m}$ band, large discrepancies are also found for extended sources, as expected (see Sect. 2.4).

\subsection{The infrared color-color diagrams}

The use of the $([12]-[25],[25]-[60])$ diagram to probe the circumstellar shells surrounding late-type stars was first demonstrated by Hacking et al. (1985) and by van der Veen \& Habing (1988, VH). VH defined various regions (labelled I to VII) corresponding to circumstellar shells with relatively homogeneous properties. Figure 2 presents the ([12] - [25], [25] - [60]) diagram for the sample of $\mathrm{S}$ stars defined in Sect. 2.1. In this paper, the color index $[i]-[j]$ is defined as $[i]-[j]=$ $-2.5 \log \left[\left(F(i) / F_{0}(i)\right) /\left(F(j) / F_{0}(j)\right)\right]$ (where $F(i)$ refers to the non-color corrected flux density in the IRAS band $i$, and $F_{0}(i)$ is a normalization flux density as given in the IRAS Explanatory Supplement 1988). With this normalization, black bodies in the Rayleigh-Jeans limit have a color index of 0 . The colors for stars whose IRAS flux densities show large variations are plotted as two symbols joined by a line segment.

There is a strong correlation between the position of an $\mathrm{S}$ star in the color-color diagram and the intensity of the spectral features distinctive of the $\mathrm{S}$ star class (as measured by the $\mathrm{ZrO} / \mathrm{TiO}$ or $\mathrm{C} / \mathrm{O}$ spectral indices in the spectral classification schemes devised by Keenan 1954 and Keenan \& Boeshaar 1980, respectively; both will be called "ZrO index" for simplicity in the following). In particular, $\mathrm{S}$ stars with $\mathrm{ZrO}$ indices larger than 3 are mainly found in Regions VII and VIa with a few more in Region III, while $\mathrm{S}$ stars with $\mathrm{ZrO}$ indices smaller than or equal to 2 are found mainly in Regions I and II. This segregation of $\mathrm{S}$ stars in the $([12]-[25],[25]-[60])$ diagram is a clear indication of the inhomogeneous nature of this family of peculiar red giants. In the center of Region I lie the $\mathrm{S}$ stars with photospheric colors. These are in fact the binary and Tc-poor S stars ("extrinsic" S stars) that owe their chemical peculiarities to mass transfer in a binary system (Sect. 1). Another group of weak S stars is found at the

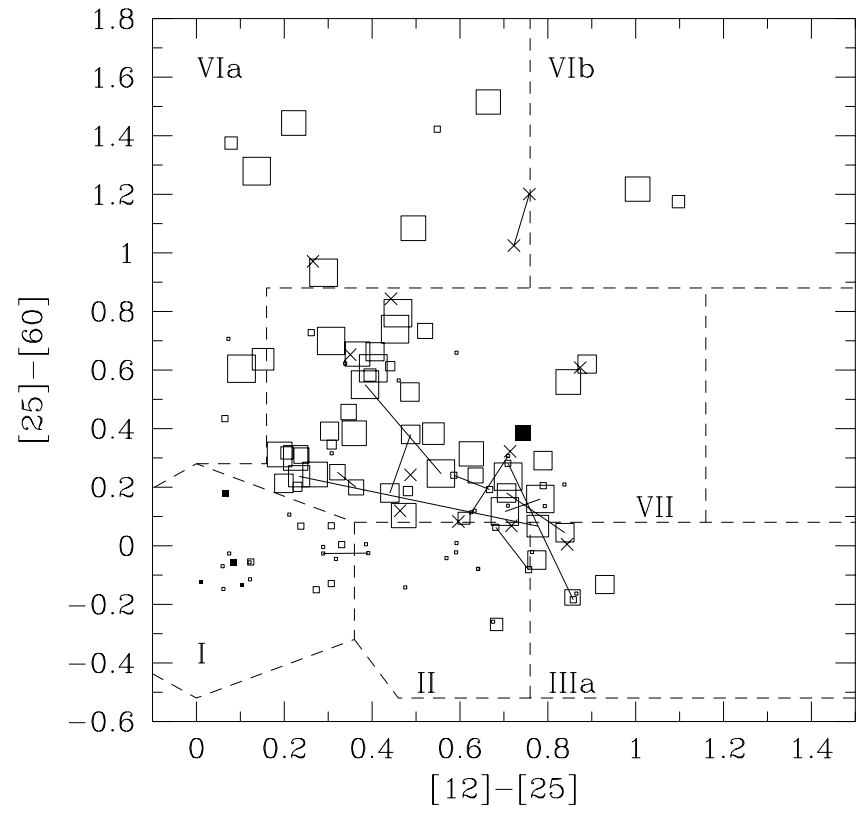

Fig. 2. The ([12] - [25], [25] - [60]) diagram for S stars. The size of the square is proportional to the $\mathrm{ZrO} / \mathrm{TiO}$ or $\mathrm{C} / \mathrm{O}$ spectral index of the S star (whichever is available), in the spectral classification scheme of Keenan (1954) or Keenan \& Boeshaar (1980), respectively. MS and SC stars were assigned classes 1 and 9, respectively. Crosses correspond to stars with no spectral type available in the GCGSS. Tc-poor S stars are represented by filled squares, and colors of variable stars measured at different epochs are connected by a line segment

boundary between Regions I and II, a zone generally devoid of stars in the $([12]-[25],[25]-[60])$ diagram (see e.g. VH, and Lewis 1989). This group includes the prototypical Tc-rich Mira S variable $\chi$ Cyg. The few S stars located in Region II, defined by $\mathrm{VH}$ as comprising O-rich stars with "young" circumstellar shells, indeed have small $\mathrm{ZrO}$ indices. Most S stars populate Region VII, where Crich circumstellar shells are found according to $\mathrm{VH}$, and it is therefore not surprising that those $\mathrm{S}$ stars have large $\mathrm{ZrO}$ indices. Finally, several stars (among which are many of type SC) are located in Regions VIa and b, many of them having conspicuously resolved circumstellar shells (see Sect. 2.4).

Several authors (see e.g. Habing 1996) have argued that the $K-[12]$ index is superior to the [12] - [25] index for tracing the mass loss rate, the focus of the present study, because of its greater wavelength range and the fact that the photosphere emits more strongly at $K$ while the shell emits more strongly at $12 \mu \mathrm{m}$. Therefore, we felt that it was more meaningful to use the $(K-[12],[25]-[60])$ diagram to define groups of S stars with homogeneous IR properties, as follows (Fig. 3):

Region A: S stars with photospheric IR colors ("extrinsic S stars");

Region B: S stars with no excesses at $60 \mu \mathrm{m}$, and small 


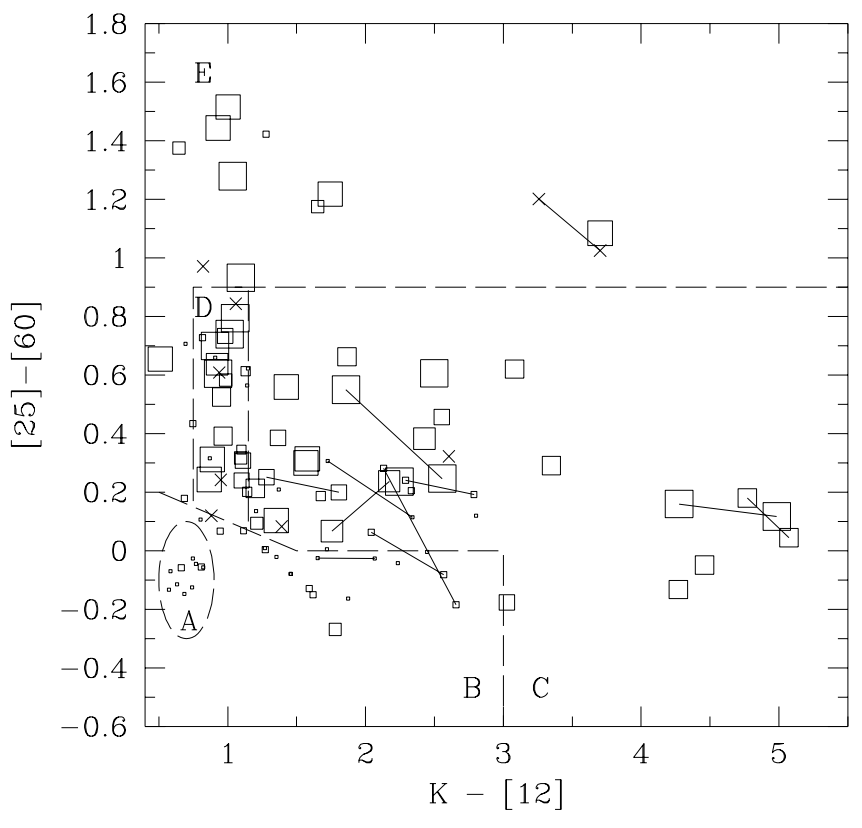

Fig. 3. Same as Fig. 2 for the $(K-[12],[25]-[60])$ diagram. Note that the $K-[12]$ index may be inaccurate for variable stars, since their $K$ and [12] magnitudes were not measured simultaneously

$\mathrm{ZrO}$ indices;

Region C: S stars with excesses in all three $12 \mu \mathrm{m}, 25 \mu \mathrm{m}$ and $60 \mu \mathrm{m}$ bands, and large $\mathrm{ZrO}$ indices;

Region D: S stars with large $\mathrm{ZrO}$ indices and $60 \mu \mathrm{m}$ excesses, but small $12 \mu \mathrm{m}$ excesses;

Region E: mainly SC stars with large $60 \mu \mathrm{m}$ excesses and often resolved shells.

The stars classified this way are plotted in the ([12] - [25], [25] - [60]) color-color diagram in Fig. 4, and it is seen that the above regions almost exactly correspond to those of $\mathrm{VH}$, with the exception of Region B which encompasses both Regions I and II, and Region VII which is a blend of Regions C and D (though stars from Region $\mathrm{D}$ are concentrated in the upper left of Region VII). The motivation for creating Region $\mathrm{D}$ is apparent in Fig. 5, which presents the distribution of the various IR spectral types (as defined by Volk \& Cohen 1989) across the $(K-$ [12], [25] - [60]) diagram. Region D differs from Region $\mathrm{C}$ in having many stars exhibiting a stellar continuum in the IR (class S) and none with silicate emission (class E). As it will be shown in Sect. 4, these features suggest that the circumstellar shells in Region D contain C-rich grains, whereas silicate grains are found in the circumstellar shells of Region C. Note that Regions $\mathrm{C}$ and D are also quite distinct in the $(K-[12],[12]-[100])$ diagram (Fig. 6).

Finally, let us remark that the different regions defined above have been denoted by uppercase letters to avoid confusion with the Regions a to d defined in Paper I from the lower-accuracy PSC flux densities. The new classification into Regions A-E is actually not very different from

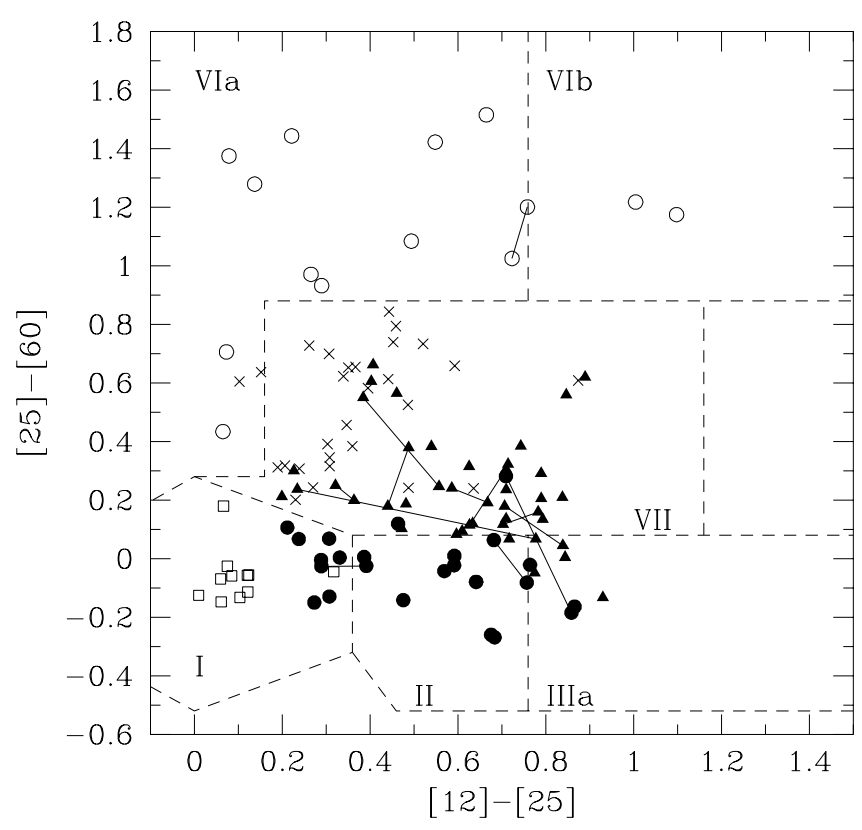

Fig. 4. Same as Fig. 2, where the stars have been drawn with a symbol corresponding to the region they belong to in the $(K-[12],[25]-[60])$ diagram (Fig. 3), as follows: open squares: Region A; filled circles: Region B; filled triangles: Region C; crosses: Region D; open circles: Region E

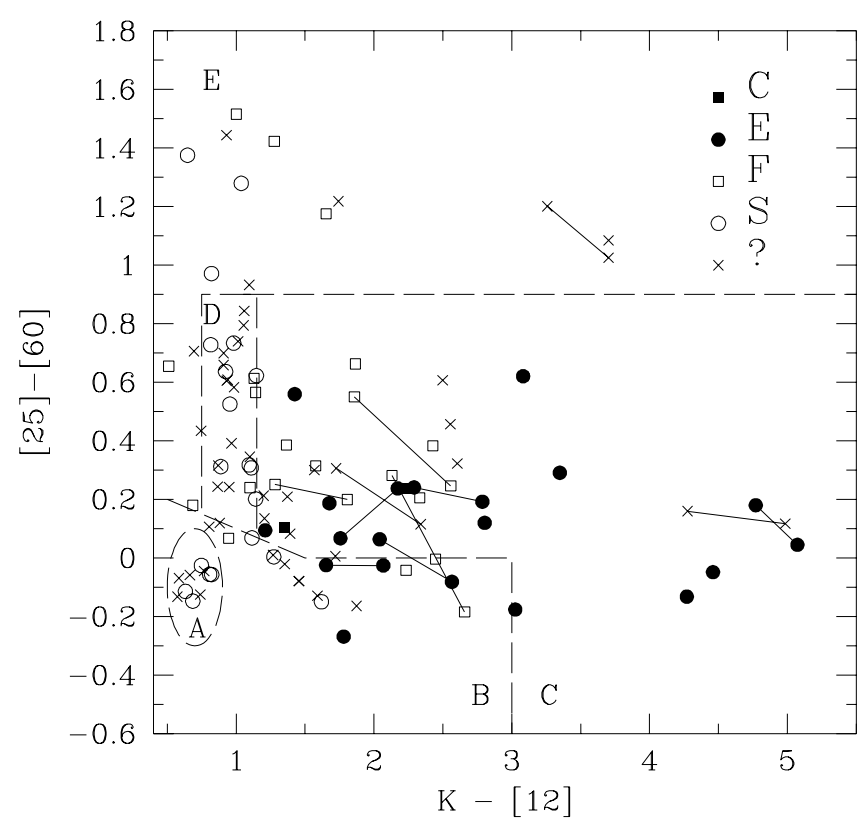

Fig. 5. Same as Fig. 3, with symbols referring to the spectral class of the IRAS low-resolution spectrum as defined by Volk \& Cohen (1989): S: stellar continuum; F: featureless spectrum; E: silicate emission; C: SiC emission; ?: not available. Spectral type assignments for S stars are from Chen et al. (1995) 


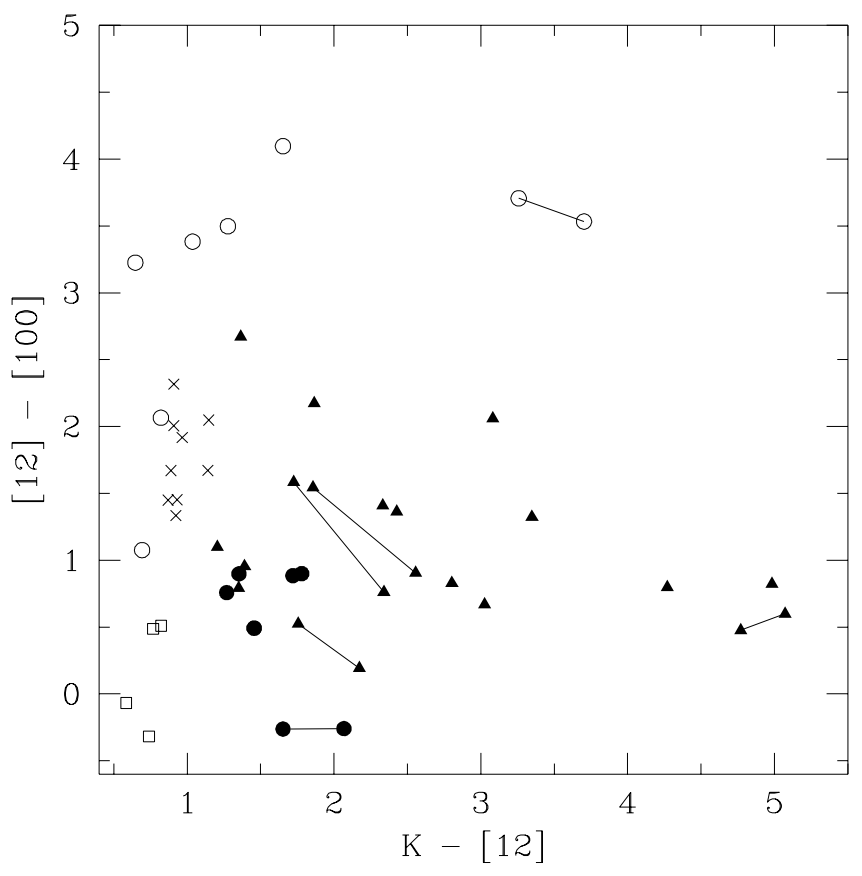

Fig. 6. The $(K-[12],[12]-[100])$ diagram. Symbols are as in Fig. 4

that in Paper I (matching uppercase with lowercase letters, and with Region d splitting into D and E), but it is clearly superior since several stars that appeared exceptional in Paper I fit well into the present classification. For instance, $\chi$ Cyg, NQ Pup and $o^{1}$ Ori were exceptional as being Tc-rich stars in Region a. With the more accurate ADDSCAN flux densities, $\chi$ Cyg moves to Region $B$ (see also Appendix A) while NQ Pup and $o^{1}$ Ori move to Region E. The only remaining outliers in this respect are HR Peg, the only Tc-rich star in Region A, and DY Gem, the only Tc-poor star not in Region A (but in C). DY Gem is however exceptional in many other respects: it is a very cool star (S8,5 corresponding to $T_{\text {eff }}=3000 \mathrm{~K}$; Smith \& Lambert 1990) and a SRa variable with a very long pe$\operatorname{riod}(1145 \mathrm{~d})$. Moreover, it has the largest [12]-[100] index among stars in Region C (see Fig. 6).

\section{4. $S$ stars with envelopes resolved at $60 \mu \mathrm{m}$}

The possibility that some S stars may have circumstellar shells resolved by IRAS is now examined. As argued by Young et al. (1993a; YPK), the $60 \mu \mathrm{m}$ band is best suited for that purpose, because it is not contaminated by Galactic cirrus emission as severely as is the $100 \mu \mathrm{m}$ band. The $60 \mu \mathrm{m}$ co-added scans were examined, and characterized as follows. First, the width of the template profile fitted to the source is compared to that expected for point sources, namely 2!.05 and 1'.44 full widths at the $25 \%$ and $50 \%$ levels, respectively (Levine et al. 1993). However, this criterion is not sensitive to resolved shells showing up as a weak plateau at the base of the profile. A simple cri- terion has therefore been devised, based on the comparison of the "peak flux density" $F_{\mathrm{p}}$ with the "zero-crossing flux density" $F_{\mathrm{z}}$ (see Sect. 2.2). These quantities are standard outputs from the ADDSCAN procedure. In the case of a point source, $F_{\mathrm{z}}$ and $F_{\mathrm{p}}$ are identical within a few times the noise, measured as the rms $\sigma$ of the residuals along the baseline outside the signal range (i.e. between 2.5 and $10^{\prime}$ from the target position in either directions) after baseline subtraction (see Sect. 2.2). In the case of an extended source, the fraction $\epsilon$ of flux density in excess of that of a point source is expressed by $\epsilon=\left(F_{\mathrm{z}}-\right.$ $\left.F_{\mathrm{p}}\right) / F_{\mathrm{p}}$. For estimating the significance of this excess, one has to be aware of the following effects. First, very bright (> $500 \mathrm{Jy}$ ) sources may have a characteristic six-pointed star shape due to reflection from the telescope secondary mirror struts. Since approximately $5 \%$ of the peak flux density may be contained in the star pattern, $F_{\mathrm{z}} / F_{\mathrm{p}}$ values of the order of 1.05 may be of instrumental origin (Levine et al. 1993). Bright sources are also affected by hysteresis in the detector which causes a trail in the signal along the scan direction in the outgoing part of the scan. This trail is easily recognized on individual scans as it causes an asymmetry in the template fit. However, if the co-added scan results from individual scans made in opposite directions, as is often the case, trails will be present in both directions and will mimick an extended plateau at the base of the profile. For bright sources ( $>100 \mathrm{Jy}$ ), this effect may thus also cause spurious excesses of the order of a few percent. Finally, for fainter sources, an inhomogeneous background may also cause spurious detections. In this case, a way to estimate the significance of the excess $\epsilon$ is to compare it with the inverse signal-tonoise ratio $1 / S N R=\sigma / F_{\mathrm{p}}$, which is nothing more than the relative flux density excess expected from the background noise. The significance, or "quality factor" $Q F$, of the observed flux density excess can then be expressed as $Q F \equiv \epsilon F_{\mathrm{p}} / \sigma=\epsilon S N R$, so that $Q F>5$ for a detection at the $5 \sigma$ level. Figure 7 presents the ratio $F_{\mathrm{z}} / F_{\mathrm{p}}$ vs. $S N R$ for $\mathrm{S}$ stars not confused by nearby sources, and several stars with $Q F>5$ are found. One has to be aware, however, that the co-addition process will be mostly effective in lowering the baseline noise far away from the target, where scans with different orientations sample different regions of the sky. But since they all intersect approximately on the target, the noise-averaging process will not be as effective on the target. A criterion based on the absolute noise level present on-target has therefore been considered as well, by requiring that, to be considered significant, an excess $F_{\mathrm{z}}-F_{\mathrm{p}}$ should not be smaller than some given threshold value of the order of the background fluctuations in the $60 \mu \mathrm{m}$ band. It was found that meaningful results are obtained by adopting $0.3 \mathrm{Jy}$ as the typical background fluctuation on-target, combined with a quality factor of 5 . In Fig. 7 , sources satisfying the $F_{\mathrm{z}}-F_{\mathrm{p}}>0.3 \mathrm{Jy}$ criterion have been represented by open squares. Since very few square symbols are located below the dashed line in 
Fig. 7, both criteria are fulfilled simultaneously for most of the stars, and thus provide consistent conclusions regarding the resolved nature of these sources.

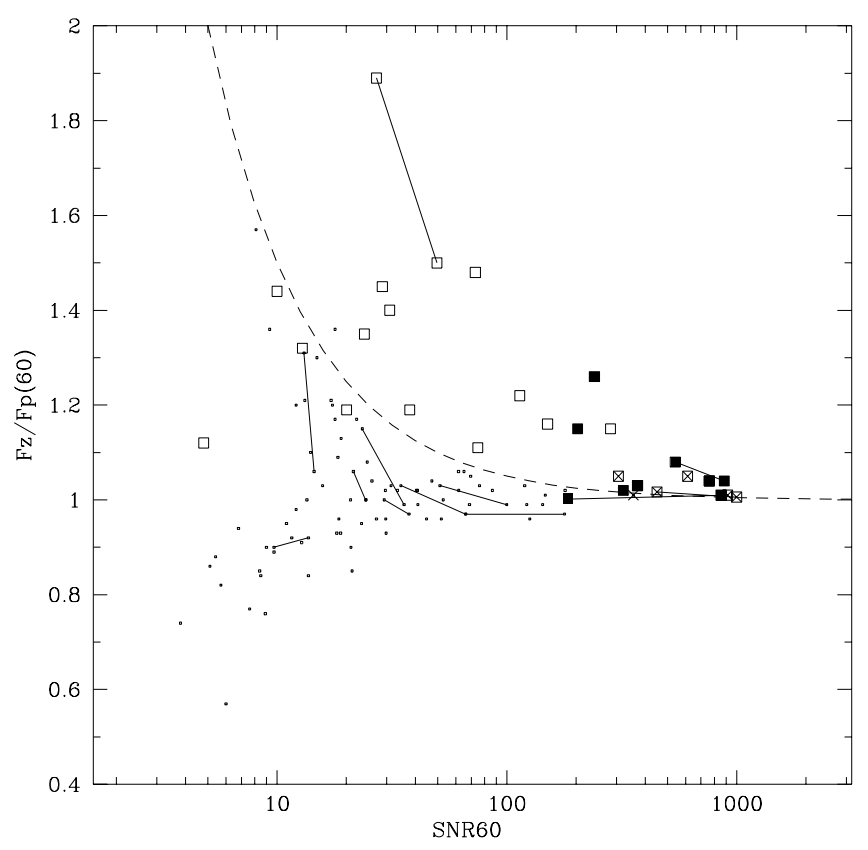

Fig. 7. The ratio $F_{\mathrm{z}} / F_{\mathrm{p}}$ between the "zero-crossing flux density" and the "peak flux density" in the $60 \mu \mathrm{m}$ band, vs the $\mathrm{S} / \mathrm{N}$ ratio along the baseline (see text for details). Stars lying above the dashed line have a flux density excess in the $60 \mu \mathrm{m}$ band with a quality factor larger than 5 . Stars with a flux density excess above the $0.3 \mathrm{Jy}$ threshold are represented by squares. Squares lying above the dashed line thus satisfy both criteria defined in the text and are probably truly resolved sources. Filled squares and crosses correspond to sources flagged by YPK as extended or non-extended, respectively, at $60 \mu \mathrm{m}$. Observations of the same star at two different epochs are connected by a line segment. Sources confused with a nearby source are not plotted

Sources flagged as extended at $60 \mu \mathrm{m}$ by the above criteria are listed in Table 2. Columns 1, 2 and 3 give the IRAS name, the variable star name and variability type when available, respectively. The zero-crossing flux density $F_{\mathrm{z}}$ is in Col. 4. Column 5 lists the flux density ratio $F_{\mathrm{z}} / F_{\mathrm{p}}$, and Cols. 6 and 7 the full widths $W 25$ and $W 50$ at the $25 \%$ and $50 \%$ levels, respectively, to be compared with 2 '.05 and 1'.44 for a point source (Levine et al. 1993). The quality factor $Q F$ is listed in Col. 8. Mass loss rates and wind terminal velocities are given in Cols. 9 and 10 (see Sect. 5).

A similar search for late-type giants with resolved shells was performed by Young et al. (1993a,b). These authors used a more sophisticated method based on the possibility of successfully fitting the signal by a point source surrounded by a circumstellar shell having "reasonable" properties. Our simpler approach has the advantage of being applicable to fainter stars, and several have indeed been detected, as seen in Fig. 8. However, for bright sources, our method is more vulnerable to spurious detections due to hysteresis (YPK used only the scan data taken prior to passing over the source).

As can be seen in Table 2 (in Col. 11, YPK + and YPK - denote sources flagged by YPK as extended or not at $60 \mu \mathrm{m}$, respectively), the two methods give conflicting results for four stars, R And, S Cas, W Aql and $\mathrm{T}$ Cet among the 11 bright objects common to the two samples. In the first three cases, our detection is probably an artefact due to detector hysteresis, since individual (as opposed to co-added) scans show an extended tail only on the side posterior to the passage over the source (see Fig. 10 below). The situation is less clear for T Cet, as its $100 \mu \mathrm{m}$ profile is wider than the point-source template, suggesting that this source may be truly extended.

Figure 8 presents the flux density excess $F_{\mathrm{z}} / F_{\mathrm{p}}$ vs. $F(2.2)$, the flux density at $2.2 \mu \mathrm{m}$, and reveals that the properties of the resolved shells in Regions B and C are very different from those of Regions $\mathrm{D}$ and $\mathrm{E}$. In Regions $\mathrm{B}$ and $\mathrm{C}$, the flux density excess is of the order of a few percent, with a maximum of $15 \%$ for Y Lyn. Because the flux density excess is so small, the envelopes around stars in Regions $\mathrm{B}$ and $\mathrm{C}$ can be resolved only for the stars closest to the sun [i.e. with large $F(2.2)$ ], as shown by Fig. 8 . By contrast, stars in Regions D and E have much larger flux density excesses at $60 \mu \mathrm{m}$, which make them detectable at much lower total flux density levels. Stars in Regions B and $\mathrm{C}$ also differ markedly from those in Regions $\mathrm{D}$ and $\mathrm{E}$ as far as the [60] - [100] index is concerned (Fig. 9; see also Fig. 6): the resolved envelopes in Regions D and E go along with large $100 \mu \mathrm{m}$ excesses, suggestive of cool dust in detached envelopes, contrary to the situation prevailing in Regions $B$ and $C$ (the only exception being $T$ Sgr, which appears as a border case between $\mathrm{C}$ and $\mathrm{D})$. In the case of RZ Sgr, which is extended in both the $60 \mu \mathrm{m}$ (YPK) and $100 \mu \mathrm{m}$ bands (according to the IRAS Small Scale Structure Catalogue 1985), an optical nebula has even been reported by Whitelock (1994).

In Region E, $o^{1}$ Ori and S929 do not follow the general trend. In those cases, there may be a confusing background source responsible for the strongly asymmetric $60 \mu \mathrm{m}$ profile (see Fig. 10), as the $100 \mu \mathrm{m}$ profile is offset by about $1^{\prime}$ in the direction of the $60 \mu \mathrm{m}$ asymmetry. The extension observed for S929 may be real; its [60] - [100] color index changed by only $2 \%$ between the two IRAS observations, while the $60 \mu \mathrm{m}$ flux density changed by $20 \%$. This color stability is observed in other variable IRAS sources like $\chi$ Cyg (see Appendix A), but would probably not be preserved if the excess flux density were due to a background source.

Finally, the flux density excess in Regions D and E is associated with a widening of the whole $60 \mu \mathrm{m}$ (and sometimes $100 \mu \mathrm{m}$ ) source profile, whereas the excess for stars in Regions B and C is caused by a weak plateau at the 


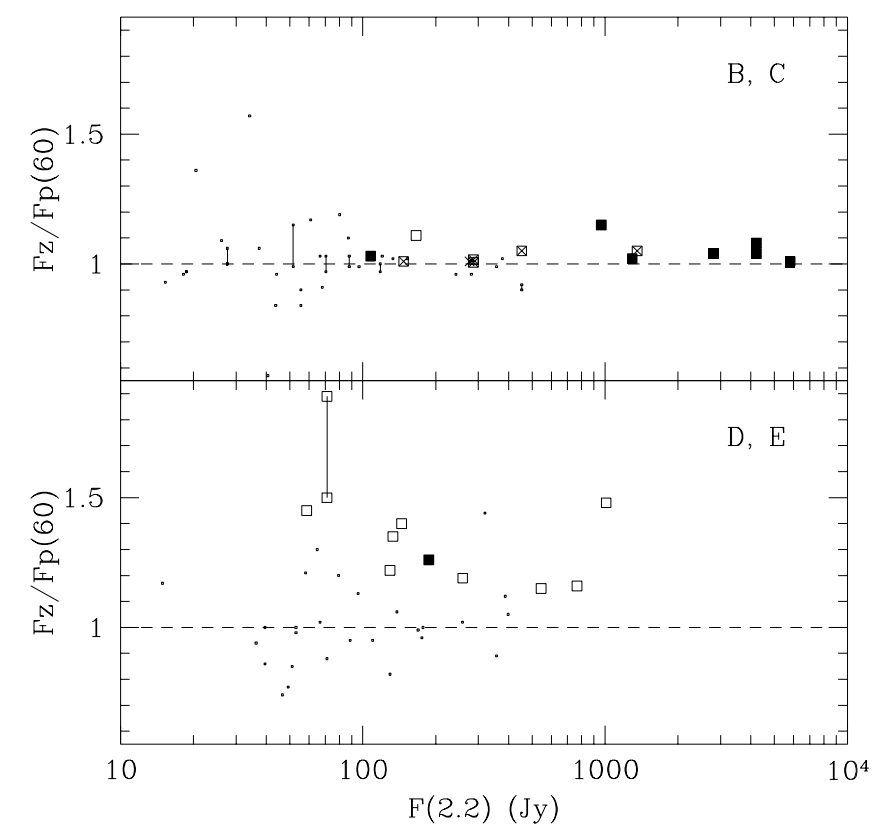

Fig. 8. Flux ratio $F_{\mathrm{z}} / F_{\mathrm{p}}$ vs. $F(2.2)$. Symbols are as in Fig. 7, except that large squares now denote stars fulfilling simultaneously our two criteria for extended envelopes. Stars have been separated according to Regions B, C (upper panel) and D, E (lower panel)

base of the profile, as apparent in Fig. 10. For the resolved shells in Regions D and E, the full widths at the $25 \%$ and $50 \%$ flux density levels (Table 2) are indeed much larger than those expected for a point source. HD 191630 and RZ Sgr are listed in the IRAS Small Scale Structure Catalogue as extended at $60 \mu \mathrm{m}$ and $100 \mu \mathrm{m}$, respectively. In fact, this distinctive feature of the resolved shells in Regions $\mathrm{D}$ and $\mathrm{E}$ has been used to include in Table 2 two stars (BI And and AA Cam) with wide profiles, despite quality factors $Q F<5$ that would normally not qualify them. However, these are distant stars with small $60 \mu \mathrm{m}$ flux densities, so that the $F_{\mathrm{z}} / F_{\mathrm{p}}$ ratio cannot be determined accurately (and is therefore not listed in Table 2).

Stars with resolved shells are represented as black dots in the $(K-[12],[25]-[60])$ diagram (Fig. 11). This figure illustrates the relative contribution of the extended shell to the $[25]-[60]$ index: a line segment joins the $[25]-$ [60] indices computed from the "zero-crossing" flux density $F_{\mathrm{z}}$ at $60 \mu \mathrm{m}$ (measuring the combined contributions of the star and its resolved shell) and from the "template" flux density $F_{\mathrm{t}}$, which is assumed to be a rough measure of the stellar contribution alone (represented by a black dot in Fig. 11). Although that assumption is certainly a very rough one, it is not totally unreasonable as stars from Region E now move down, and most reach Regions B, C and $\mathrm{D}$ when adopting $F_{\mathrm{t}}$ instead of $F_{\mathrm{z}}$ to represent the $60 \mu \mathrm{m}$ photospheric flux density. Some stars, however, do not quite leave Region E, presumably because the point

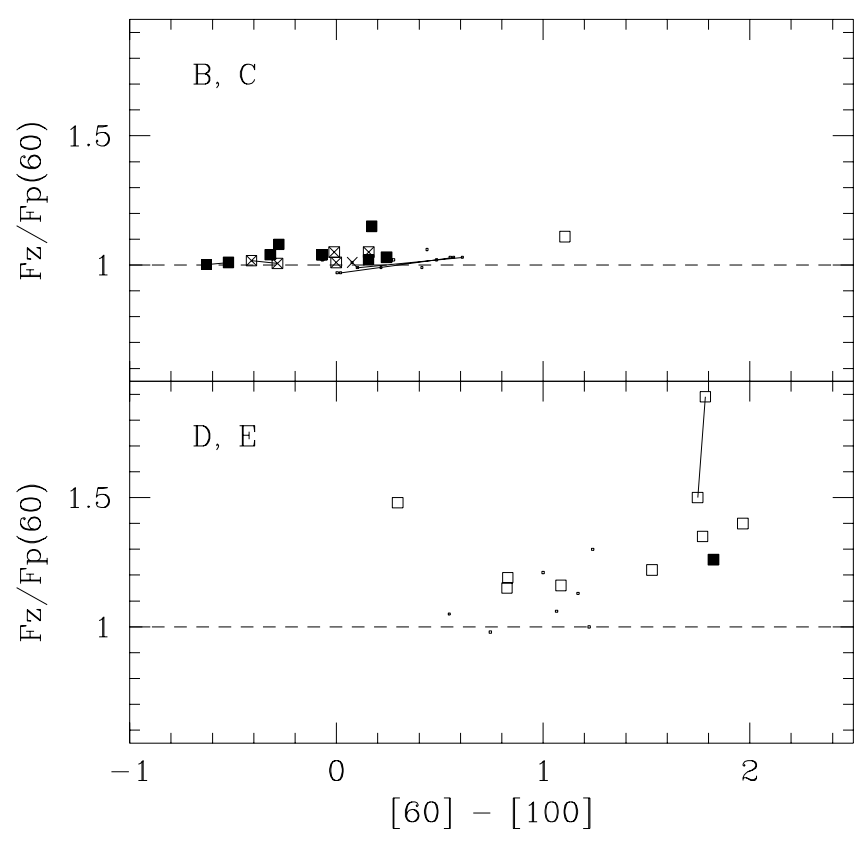

Fig. 9. Same as Fig. 8 for $F_{\mathrm{z}} / F_{\mathrm{p}}$ vs. [60] $-[100]$

Regions B,C
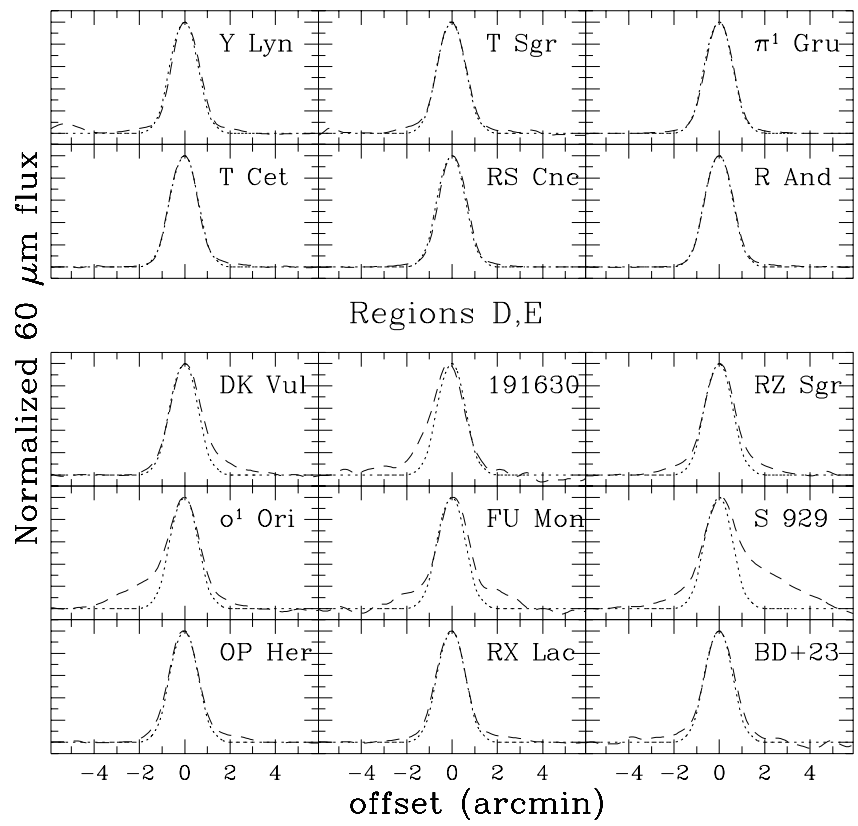

Fig. 10. The $60 \mu \mathrm{m}$ co-added, spline-interpolated scans (dashed lines) for all stars with a possibly resolved shell in Regions D - E, and for selected cases in Regions B - C (see Table 2). The dotted line is the template $60 \mu \mathrm{m}$ profile as provided by IPAC. Note how larger the deviations from the template are in Regions D - E as compared to Regions B - C. In the latter case, the deviation of the observed profile from the template profile is due to a weak extended tail. Such detections are therefore vulnerable to detector hysteresis (see text) 


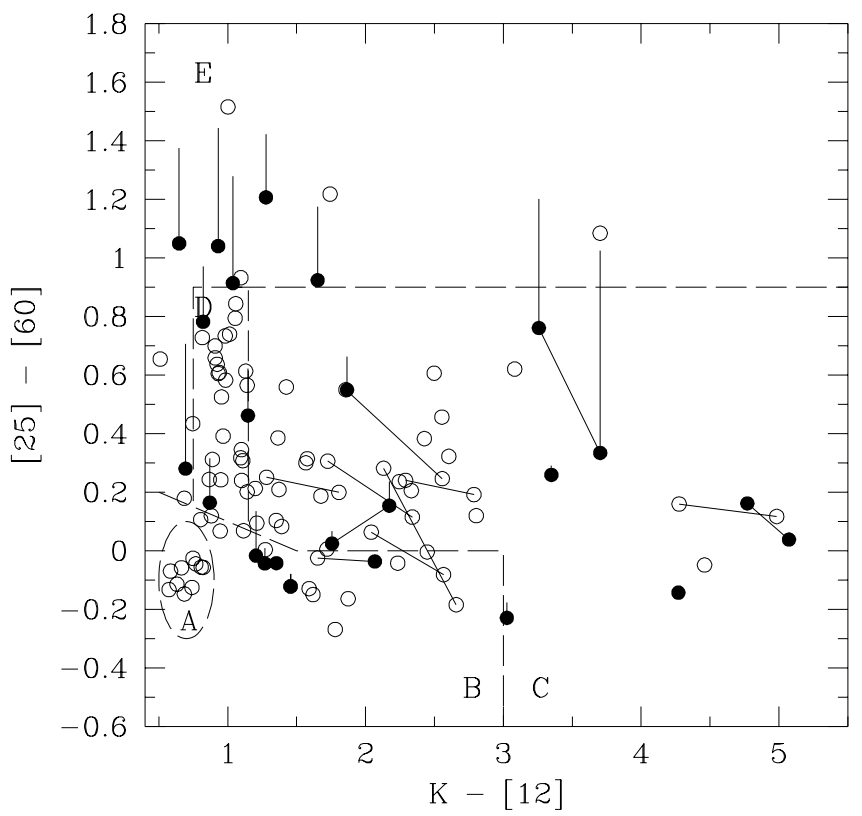

Fig. 11. Position of the sources with resolved shells (filled circles) in the the $(K-[12],[25]-[60])$ diagram. The filled circles represent the color computed from the "template" flux density $F_{\mathrm{t}}$ at $60 \mu \mathrm{m}$, i.e. they roughly correspond to the flux density of the star alone. The upper end of the vertical segment is located at the color computed from the "zero-crossing" flux density $F_{\mathrm{z}}$ at $60 \mu \mathrm{m}$, i.e. the shell + star color

source fitting yielding $F_{\mathrm{t}}$ does not entirely remove the contribution of the resolved shell in those cases.

Especially interesting is the fact that Y Lyn and OP Her move from Regions $\mathrm{C}$ and $\mathrm{D}$, respectively, to Region $\mathrm{B}$, which is well in line with their small $\mathrm{ZrO}$ index (see Fig. 3 where they appear as outliers). With this adjustment, all three SRc variables in our sample (RS Cnc, Y Lyn and T Cet) now belong to Region B, and moreover have resolved shells (tentative in the case of $\mathrm{T}$ Cet). As noted by Young et al. (1993a,b) and Habing (1996), resolved shells are a common property of semi-regular variable stars.

Finally, it should be mentioned that several of the stars with a shell resolved by IRAS turn out to have an extended CO shell as well, as derived in Sect. 5.3 from the modelling of the $\mathrm{CO}$ data. The inferred radius of the $\mathrm{CO}$ shell (Table 6) is larger than $10^{\prime \prime}$ for $\chi$ Cyg $\left(18^{\prime \prime}\right)$, W Aql $\left(23^{\prime \prime}\right), \pi^{1}$ Gru $\left(15^{\prime \prime}\right)$ and FU Mon $\left(60^{\prime \prime}\right)$.

\section{Masers in $\mathbf{S}$ stars}

This section presents data collected from the literature on $\mathrm{SiO}, \mathrm{OH}$ or $\mathrm{H}_{2} \mathrm{O}$ maser emission from $\mathrm{S}$ stars (relying on the compilation of Benson et al. 1990 for the earlier literature). These data are summarized in Table 3 . The detection of $\mathrm{SiO}, \mathrm{OH}$ or $\mathrm{H}_{2} \mathrm{O}$ maser emission is a clear indication that the circumstellar shell is oxygen-rich (see

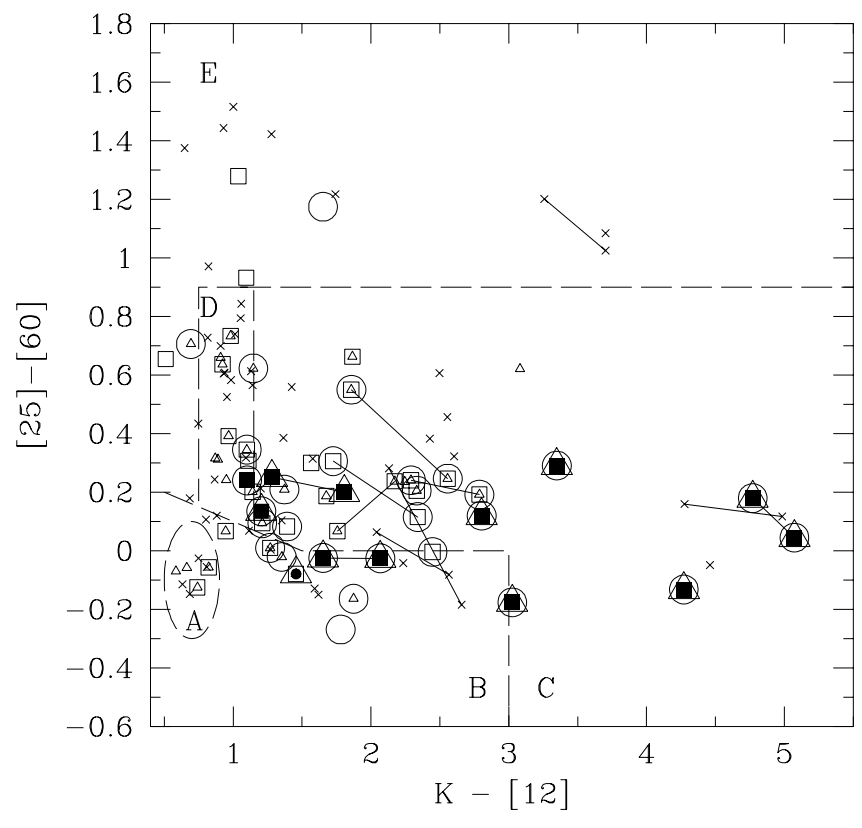

Fig. 12. Location of the maser sources in the $(K-[12]$, [25] [60]) diagram. Positive detections correspond to filled symbols, non-detections to open symbols, and stars not probed for maser emission to crosses. Squares stand for $\mathrm{SiO}$ masers, triangles for $\mathrm{H}_{2} \mathrm{O}$ masers and circles for $\mathrm{OH}$ masers

e.g. Deguchi et al. 1989, Lewis 1996), thus providing an important constraint on the chemical nature of the dust grains (see Sect. 4). In Table 3, "Y" or "N" in the $\mathrm{SiO}$, $\mathrm{OH}$ or $\mathrm{H}_{2} \mathrm{O}$ columns means that the corresponding maser is or is not present, respectively, whereas a dash indicates that the maser has not been searched for in a given star.

The masers which are found in $\mathrm{S}$ stars are mainly $\mathrm{SiO}$ masers forming in the densest part of the circumstellar envelope near the stellar photosphere. $\mathrm{OH}$ maser emission has been detected in RS Cnc by Rudnitskij (1976) but not by Kolena \& Pataki (1977), though the latter authors probed a different transition from that $(1667 \mathrm{MHz})$ detected by Rudnitskij (1976).

The $\mathrm{S}$ stars with $\mathrm{SiO}$ maser emission lie in the lower part of Region C, with some overlap with Regions B and D (Fig. 12). As expected, the region occupied by the masers exactly matches the region delineated by the stars showing the $9.7 \mu \mathrm{m}$ silicate feature in emission (IRAS LRS class E; Fig. 5). Indeed, 6 of the $8 \mathrm{SiO}$ masers with an available LRS class are of class E, the only exceptions being R Lyn and EP Vul (class F). On the other hand, no maser emission is observed for stars in Regions D and E (with the exception of EP Vul, but that star lies very close to the boundary with Region C). 


\section{A simple model of dusty circumstellar shells}

\subsection{Ingredients of the model}

In order to relate the diversity of IR color indices observed among S stars to the underlying physical parameters, synthetic IR color indices of a star embedded in a circumstellar shell have been computed for various input parameters. In our simple model, the star is assumed to radiate as a black body at a temperature $T_{\text {eff }}$. The mass-losing star is surrounded by a spherically-symmetric dust shell extending from $r_{\text {in }}$ to $r_{\text {out }}$, with density decreasing as $r^{-2}$, $r$ being the distance from the central star. This is equivalent to assuming a steady mass-loss rate $\dot{M}$ at constant outflow speed. The inner radius of the dust shell must be larger than or equal to the radius where grains start condensing (i.e., to the radius where the shell temperature drops below $1300 \mathrm{~K}$ for silicates, or $1500 \mathrm{~K}$ for graphite and amorphous carbon). The shell outer radius is chosen such that $r_{\text {out }} / r_{\text {in }}=10^{4}$, which ensures that the color indices of the shell have reached an asymptotic value. A roughly logarithmic radial mesh is defined in the dust envelope so that each shell is optically thin. In each shell, the grains are assumed to be in thermal equilibrium, so that the energy absorbed by the grains exactly balances the energy re-emitted. At the inner boundary, the radiation field is that of a black body of temperature $T_{\text {eff }}$. The model IRAS flux densities are calculated from the emergent spectrum by convolving it with the IRAS filter bandpass (IRAS Explanatory Supplement, 1988). The main shortcoming of the code is the neglect of the scattering contribution, since only absorption is taken into account.

Three types of dust grains have been considered: silicates (with a specific mass of $3.5 \mathrm{~g} \mathrm{~cm}^{-3}$ ), graphite (with a specific mass of $2.25 \mathrm{~g} \mathrm{~cm}^{-3}$ ) and amorphous carbon (with a specific mass of $1.85 \mathrm{~g} \mathrm{~cm}^{-3}$ ). The grain radius is $0.2 \mu \mathrm{m}$ in all cases. The absorption coefficients as a function of wavelength are taken from Draine \& Lee (1984) and Draine (1985) for silicates and graphite. For amorphous carbon, the absorption coefficients have been generated with the usual Mie formulae using the optical constants provided by Rouleau \& Martin (1991). At wavelengths $\lambda>50 \mu \mathrm{m}$, the spectral index of the emissivity coefficient has been taken equal to -2 for graphite grains, and to -1.5 for amorphous-carbon and silicate grains (Ivezić \& Elitzur 1995).

\subsection{Synthetic color indices}

The results of this model are presented in Fig. 13 for dust shells made of either silicate grains, graphite grains or amorphous carbon grains. Constant dust mass loss rates of $10^{-12}, 10^{-10}, 10^{-9}, 10^{-8}$ and $10^{-7} M_{\odot} \mathrm{y}^{-1}$ with a wind velocity of $14 \mathrm{~km} \mathrm{~s}^{-1}$ have been adopted. The shell inner radius is set by the grain-condensation temperature, so that the dust shells computed in Fig. 13 are not "de- tached" (in the sense of Willems \& de Jong 1988). The central star has been assigned effective temperatures $T_{\text {eff }}$ of $4000 \mathrm{~K}$ (solid line) and $3000 \mathrm{~K}$ (dashed line), and a luminosity $L=5000 L_{\odot}$; these parameters turn out to have little impact on the shell colors.

In the $([12]-[25],[25]-[60])$ and $(K-[12]$, $[25]$ - [60]) diagrams, the silicate track is quite distinct from the graphite or amorphous-carbon tracks. The graphite and amorphous-carbon tracks go directly from Region I to upper VII (i.e., from A to D and upper C), whereas the silicate track goes from I to II and IIIa (i.e., from $\mathrm{A}$ to $\mathrm{B}$ and lower $\mathrm{C}$ ). These differences observed in Fig. 13 between carbon- and oxygen-rich shells must be related to the different emissivities of silicate and carbonaceous grains in the IRAS bands, as discussed by Ivezić \& Elitzur (1995).

The tracks for carbonaceous and silicate grains predicted by this simple model outline the segregation observed in the color-color diagrams between $\mathrm{S}$ stars with an oxygen-rich shell, as indicated by the $9.7 \mu \mathrm{m}$ silicate feature (IRAS LRS class E), and S stars with featureless IR spectra (IRAS LRS class S - "stellar" - or F "featureless"). It is important to note here that featureless spectra are indeed predicted for carbonaceous grains (Ivezić \& Elitzur 1995).

The data on maser emission collected in Sect. 3 are compatible with this segregation. Those $\mathrm{S}$ stars which are $\mathrm{SiO}$ or $\mathrm{OH}$ masers (Table 3) must have an oxygen-rich circumstellar environment, and lie indeed close to the silicate track (Fig. 12). On the contrary, no $\mathrm{SiO}, \mathrm{OH}$ or $\mathrm{H}_{2} \mathrm{O}$ maser emission has been detected for $\mathrm{S}$ stars in Regions D and upper $\mathrm{C}$, along the tracks corresponding to carbonaceous grains. Those $\mathrm{S}$ stars might therefore possibly be surrounded by C-rich circumstellar shells, especially since they occupy the same region of the color-color diagram as the optical carbon stars (Chan \& Kwok 1988).

However, the above picture is not totally satisfactory, since (i) the $\mathrm{S}$ stars with silicate emission are actually located in between the silicate and carbonaceous tracks, (ii) many stars in Regions D and E have large $60 \mu \mathrm{m}$ excesses that cannot be reproduced by the tracks displayed in Fig. 13, (iii) at least one star (R Gem) moves from the region of silicate dust (lower $\mathrm{C}$ ) into the region of carbonaceous dust (upper C) during its variability cycle, and (iv) the photosphere of $\mathrm{S}$ stars is oxygen-rich, so that their circumstellar shell may be expected to be oxygen-rich as well. The first two mismatches are in fact not specific to $\mathrm{S}$ stars, and possible solutions were already suggested by Ivezić \& Elitzur (1995). They include either (a) invoking a mixture of silicate and carbonaceous grains (see however the discussion of Sect. 3 on maser emission), (b) decreasing the spectral index of the emissivity at long wavelengths to values smaller than -1.5 , or (c) considering detached envelopes in the sense advocated by Willems \& de Jong (1988). 


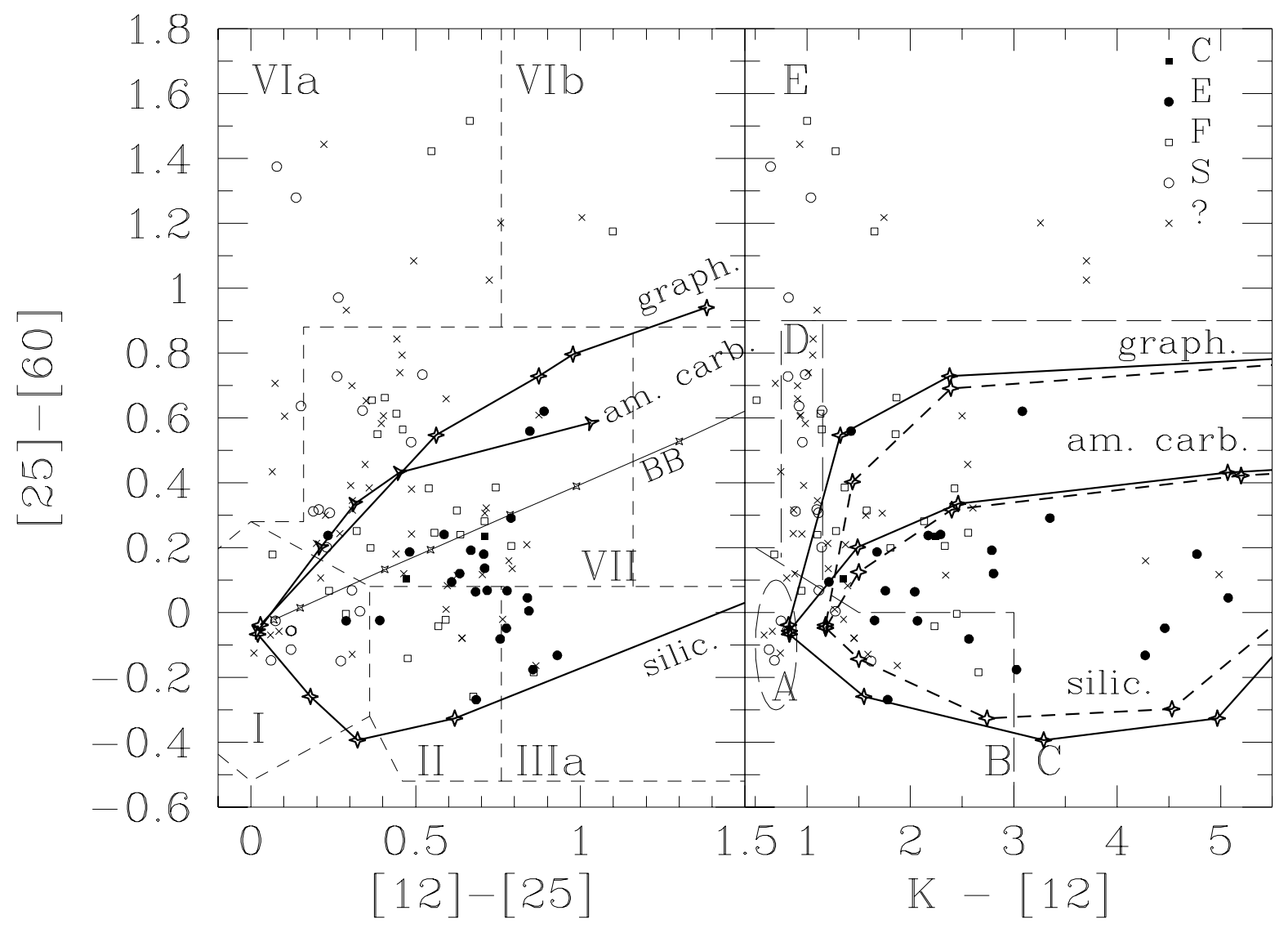

Fig. 13. Left panel: The ([12] - [25], [25] - [60]) colors predicted for circumstellar shells (with $\left.r_{\text {out }} / r_{\text {in }}=10^{4}\right)$ made of silicate grains, graphite grains or amorphous-carbon grains, surrounding a star with $L=5000 L_{\odot}$ and $T_{\text {eff }}=4000 \mathrm{~K}$ (solid lines) or $3000 \mathrm{~K}$ (dashed lines). The diamonds along the curves correspond to dust mass loss rates of $10^{-12}, 10^{-10}, 10^{-9}, 10^{-8}$ and $10^{-7} M_{\odot} \mathrm{y}^{-1}$ (from left to right), with a wind velocity of $14 \mathrm{~km} \mathrm{~s}^{-1}$. Black bodies fall along the central straight line labelled BB. Right panel: same as left for $(K-[12],[25]-[60])$

As discussed in Sect. 2.4, the S stars located in Regions $\mathrm{D}$ and $\mathrm{E}$ have very specific properties that may help to identify the origin of their large $60 \mu \mathrm{m}$ excess. First, the prototypical SC stars GP Ori and FU Mon located in Region $\mathrm{E}$ have a $\mathrm{C} / \mathrm{O}$ ratio equal to unity to within $1 \%$ (Dominy et al. 1986). Similarly, TT Cen is a rare CS star which exhibits $\mathrm{ZrO}$ bands at some times and $C_{2}$ bands at other times (Stephenson 1973). According to Stephenson (1973), these variations are probably caused by temperature changes in an atmosphere with a $C / O$ ratio very close to unity, or perhaps even to a secular change in the atmospheric $\mathrm{C} / \mathrm{O}$ ratio. Most of the stars in Regions $\mathrm{D}$ and $\mathrm{E}$ are actually $\mathrm{SC}$ stars or at least $\mathrm{S}$ stars with a large $\mathrm{C} / \mathrm{O}$ spectral index. Finally, several stars in Region E have very extended shells that are resolved at $60 \mu \mathrm{m}$ and, in one case (RZ Sgr), visible in the optical (Whitelock 1994). All these properties point towards these stars being in a very rare and short-lived evolutionary phase. Based on this evidence, we suggest that the large $60 \mu \mathrm{m}$ excess of SC stars populating Regions D and E finds a natural explanation in the much debated concept of interrupted mass loss first proposed by Willems \& de Jong (1988). The cessation of mass loss when $\mathrm{C} / \mathrm{O}$ gets close to unity (because all $\mathrm{C}$ and $\mathrm{O}$ atoms are then locked into the $\mathrm{CO}$ molecule instead of being involved in dust-forming molecules) causes the dust shell to detach from its parent star, and to cool down as it expands into the interstellar medium without being replenished at its inner side. As shown by Willems \& de Jong (1988) and Chan \& Kwok (1988), the colors of the dust shell then describe a counter-clockwise loop in the color-color diagram, starting from the region of stars with silicate emission and ending close to the photospheric point (Region A) when the shell has dissolved into the interstellar medium, after passing through Region E. The mass loss resumes when $\mathrm{C} / \mathrm{O}$ reaches values above unity, and the star then enters the region of heavily-obscured infrared carbon stars (Chan \& Kwok 1988; lower VII in Fig. 13). The SC stars found in that region (RZ Peg, UY Cen and BH Cru, a sister case of TT Cen uncovered by Lloyd Evans 1985) may actually be on the lower part of that loop. The application of the Willems \& de Jong scenario (implying that the loop described in the 
color-color diagram corresponds to a brief evolutionary phase) to the rare, supposedly short-lived SC phase would thus not face the difficulty of inconsistent time scales generally used to argue against it (e.g., Zuckerman \& Maddalena 1989). Further support for this idea comes from the peculiar $\mathrm{CO}$ line profiles observed for many of the stars populating Region E (FU Mon: see Fig. 15 below; DK Vul, RZ Sgr, TT Cen and UY Cen: Sahai \& Liechti 1995), as discussed in Sect. 6.3.

\section{Molecular line data and mass loss rates}

\subsection{New CO data}

Observations of the $\mathrm{CO}(2-1)$ line at $230 \mathrm{GHz}$ and of the $\mathrm{CO}(3-2)$ line at $345 \mathrm{GHz}$ were made in January 1996 and January 1997 with the $10.4 \mathrm{~m}$ telescope of the Caltech Submillimeter Observatory (CSO) on Mauna Kea, Hawaii. The CSO is equipped with SIS junction receivers cooled to liquid helium temperatures. The effective single-sideband system temperatures for these observations, including the effects of atmospheric emission and absorption, were about $500 \mathrm{~K}$ and $800 \mathrm{~K}$ at 230 and $345 \mathrm{GHz}$, the telescope halfpower beamwidths were respectively $30^{\prime \prime}$ and $20^{\prime \prime}$ and the main-beam efficiencies $76 \%$ and $65 \%$.

The spectra were observed using two 1024 channel acousto-optic spectrographs (AOS) simultaneously. The first has a total bandwidth of $\sim 500 \mathrm{MHz}\left(\sim 800 \mathrm{~km} \mathrm{~s}^{-1}\right.$ at $230 \mathrm{GHz})$ and a velocity resolution of $\sim 1 \mathrm{~km} \mathrm{~s}^{-1}$. The second has a bandwidth of $\sim 50 \mathrm{MHz}$ and a velocity resolution of $0.1 \mathrm{~km} \mathrm{~s}^{-1}$. The observations were made by chopping between the star position and an adjacent sky position, offset $90^{\prime \prime}$ in azimuth, at a rate of $1 \mathrm{~Hz}$, and consisted of pairs of chopped observations with the source placed alternately in each beam. The spectral baselines resulting from this procedure are linear to within the rms noise. The telescope pointing errors were measured by mapping the spectral line emission from a nearby CO bright star before each observation was made, and the pointing accuracy is better than $\sim 5^{\prime \prime}$ for all of the observations.

The temperature scale and atmospheric opacity were measured by chopping against a hot (room temperature) load. The line temperature was corrected for the mainbeam efficiency, and the resulting scale is the RayleighJeans equivalent main beam brightness temperature $T_{\mathrm{MB}}$, i.e. that measured by a perfect $10.4 \mathrm{~m}$ antenna above the atmosphere. The spectrometer frequency was calibrated using an internally generated frequency comb, and the velocity scale is corrected to the Local Standard of Rest (LSR).

Twelve S stars were observed at the CSO (Table 4), nine in the $\mathrm{CO}(2-1)$ line and three in the $\mathrm{CO}(3-2)$ line, and emission was detected from four (Table 5 ). The line profiles for the detected stars are shown in Fig. 14.

Table 4 lists the stars that were observed: the GCGSS number and the variable star name are in Cols. 1 and 2,
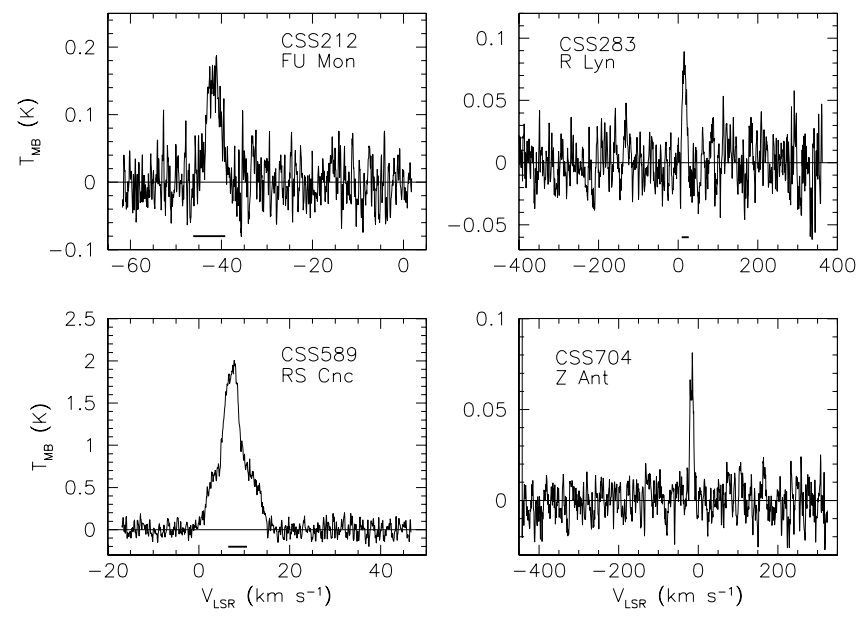

Fig. 14. $\mathrm{CO}(2-1)$ line profiles of four $\mathrm{S}$ stars observed at CSO. The abscissa is velocity with respect to the LSR and the ordinate main beam brightness temperature. The profiles for GCGSS 212 and 589 are observed with a velocity resolution of $\sim 0.1 \mathrm{~km} \mathrm{~s}^{-1}$, while GCGSS 283 and GCGSS 704 are observed with $1 \mathrm{~km} \mathrm{~s}^{-1}$ resolution. The horizontal bars show the velocity range observed in optical spectra (Table 4). There is no optical velocity available for GCGSS 704

respectively. Next is the observed position: we used positions accurate to $\sim 1^{\prime \prime}$ from the HST Guide Star Catalog and other sources (see Chen et al. 1995). Columns 5-9 list the galactic longitude and latitude, the spectral type from the GCGSS, the variable type and the period from the GCVS. Column 10 gives the stellar radial velocity with respect to the LSR measured from optical spectra; since the radial velocities of red giants vary as they pulsate, Table 4 lists the range of reported radial velocities. Finally, Col. 11 gives the channel-to-channel rms noise in the $500 \mathrm{MHz}$ AOS.

Table 5 gives the line parameters of the four detected stars: the $\mathrm{CO}(2-1)$ line flux in $\mathrm{K} \times \mathrm{km} \mathrm{s}^{-1}$, the peak line temperature, the central velocity $V_{\mathrm{c}}$ and the half-width of the line at zero power, $V_{\mathrm{e}}$, which gives the terminal wind outflow speed. These quantities are determined by fitting a parabolic line model to the data. The agreement between the optical and CO radial velocities is good.

Seven of the stars in Table 4, GCGSS 89, 117 (GP Ori), 422 (NQ Pup) 626 (FM Hya), 704 (Z Ant), 796 (HR 4755) and 803 (S UMa) have not previously been observed. We detect one of these, Z Ant. GCGSS 816 (UY Cen) is weakly detected by Sahai \& Liechti (1995, SL95) with good agreement between the $\mathrm{CO}$ and optical radial velocities. This star was not detected in the present observations, but our sensitivity is lower. The detection of circumstellar CO for this star is of particular interest, given its rare SC spectral type (see Sect. 6.3). GCGSS 149 (NO Aur) and 796 (HR 4755) were previously observed by SL95 and by Bieging \& Latter (1994, BL94); like the present observations, these did not detect $\mathrm{CO}$ emission. 
RS Cnc has been observed by many authors (see Loup et al. 1993, for example). Margulis et al. (1990) point out that the $\mathrm{CO}$ line profile for this star, as for several others, more closely resembles a triangle or gaussian in shape than the parabolic profile typical of circumstellar winds. The high velocity resolution observations in Fig. 14 show that the line profile actually consists of two parabolic components of different widths centered at the same velocity. The parameters for these profiles, estimated by eye, are given in Table 5 . This line shape may indicate the presence of two molecular winds. Such line profiles have been seen for several other stars (e.g. Margulis et al. 1990; SL95; Kahane \& Jura 1996; Knapp et al. 1997a) and may be quite common.

GCGSS 283, R Lyn, has previously been detected by BL94, and the data in Table 5 are in good agreement. FU Mon (GCGSS 212) was detected by SL95, who observe two narrow features at -44 and $+14 \mathrm{~km} \mathrm{~s}^{-1}$ which they attribute to the blue- and red-shifted components of an expanding circumstellar shell. However, the optical radial velocity of the star is about $-43 \mathrm{~km} \mathrm{~s}^{-1}$ (Table 4), and further, the narrow component at $+14 \mathrm{~km} \mathrm{~s}^{-1}$ is likely to be interstellar, as shown by our observations with the $500 \mathrm{MHz}$ AOS in Fig. 15. We conclude that the emission at $-44 \mathrm{~km} \mathrm{~s}^{-1}$ (best seen on the high resolution profile in Fig. 14) is from the circumstellar envelope. The outflow speed, $3 \mathrm{~km} \mathrm{~s}^{-1}$, is very low, but similar low values are found for some other Mira and semiregular variables (Wallerstein \& Dominy 1988; Young 1995; Kerschbaum et al. 1996).

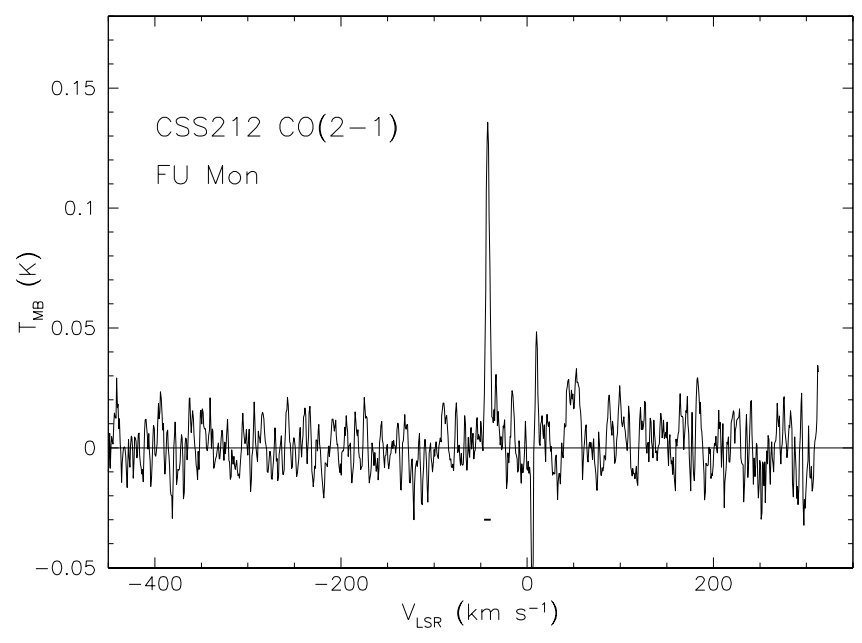

Fig. 15. Broad-band $\mathrm{CO}(2-1)$ spectrum in the direction of GCGSS 212 (FU Mon). The horizontal bar shows the range of the observed optical velocities

\subsection{CO data from the literature}

Table 6 summarizes (under the header "Observations") CO millimeter wavelength observations of S stars published since 1990, including those in the present paper. Data observed prior to 1990 can be found in Loup et al. (1993). The stars in Table 6 are grouped according to their location in the $(K-[12],[25]-[60])$ color-color diagram (see Sect. 2.3) and arranged in order of right ascension within these groups. Several observations are not listed in Table 6 because they were made at positions which are too discrepant from the optical position; the stars are GCGSS 133, GZ Peg, and T Cam.

Table 6 gives the IRAS name (an asterisk before the IRAS name refers to a note at the end of the table), the star name and the results of $\mathrm{CO}$ observations of the star: the line observed; the telescope half power beamwidth in arcseconds; the channel-to-channel rms noise in $\mathrm{K}$; the integrated line brightness in $\mathrm{K} \times \mathrm{km} \mathrm{s}^{-1}$; the peak brightness temperature in $\mathrm{K}$; the central velocity $V_{\mathrm{c}}$ with respect to the LSR; the wind outflow speed $V_{\mathrm{e}}$; and the reference. All temperatures are expressed in main-beam brightness temperature. Dashes for any of these quantities mean either that no emission was detected from the star or that the quantity in question was not quoted in the paper. Table 6 contains observations of 56 stars, with 35 detections.

To first order, the peak brightness temperatures and the integrated CO line intensities should scale inversely with the square of the telescope beamwidth, since the $\mathrm{CO}$ lines are usually fairly optically thick and the envelopes in general smaller than the beam. As Table 6 shows, this is roughly the case, and the agreement among the observations is in general good, with no serious discrepancies.

Figure 16 shows the histogram of the stellar systemic radial velocities (with respect to the LSR). The values adopted for stars with multiple observations are straight averages of the individual values. The mean velocity for $\mathrm{S}$ stars detected in $\mathrm{CO}$ is $\langle V\rangle=-9.1 \pm 5.0 \mathrm{~km} \mathrm{~s}^{-1}$ and the radial-velocity dispersion is $\sigma=25.9 \pm 4.5 \mathrm{~km} \mathrm{~s}^{-1}$. This value refers to a sample of intrinsic S stars, since no extrinsic S stars have been detected in CO. This dispersion is typical of a young-disk population (Mihalas \& Binney 1981). Such a population should have a scale-height above the galactic plane of about $200 \mathrm{pc}$, in agreement with the results of Van Eck et al. (1997).

Figure 17 shows the histogram of the wind terminal velocity $V_{\mathrm{e}}$ compared with the distributions for three other sets of molecular line observations; those for nearby oxygen-rich Mira variables (Young 1995), for semi-regular (SRa and SRb) variables (Kerschbaum et al. 1996) and for carbon stars (Olofsson et al. 1993). The outflow speeds $V_{\mathrm{e}}$ for the $\mathrm{S}$ stars are taken from Table 6 . We used average values for stars with multiple observations except for RZ Sgr (20120-4433), for which $\mathrm{CO}(1-0)$ and (2-1) observations give discrepant values (14 and $8.8 \mathrm{~km} \mathrm{~s}^{-1}$; SL95). We use the velocity derived from the $\mathrm{CO}(2-1)$ observation 


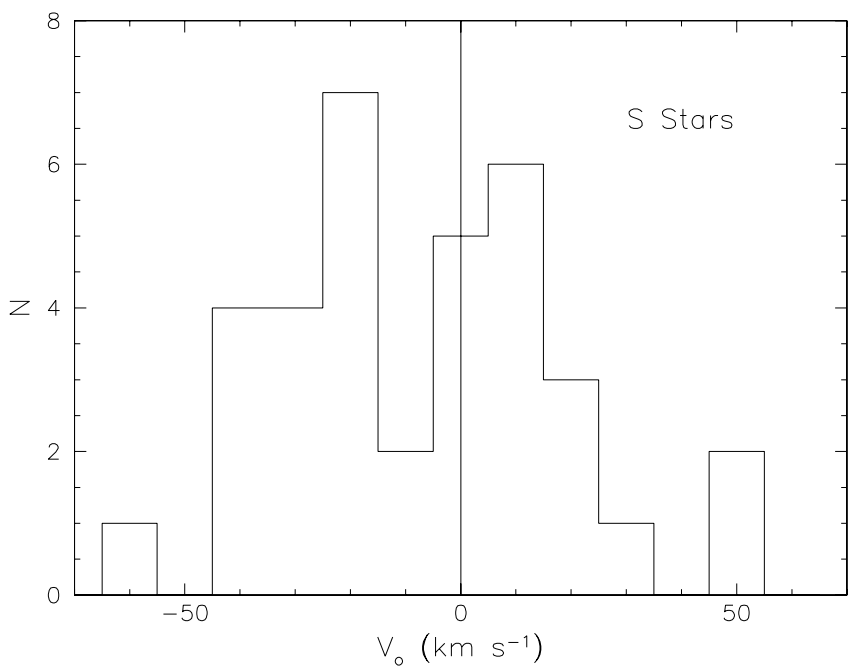

Fig. 16. Distribution of radial velocities (with respect to the LSR) from $\mathrm{CO}$ observations of intrinsic $\mathrm{S}$ stars

since this has a much higher signal-to-noise ratio, but note that the $\mathrm{CO}(1-0)$ line may really be broader; the larger telescope beam at this wavelength could be detecting gas at larger distances from the extended envelope of this star, which could have a larger outflow velocity.

Figure 17 shows that oxygen-rich Miras have the smallest outflow velocities (median $6.5 \mathrm{~km} \mathrm{~s}^{-1}$, largest value $12.7 \mathrm{~km} \mathrm{~s}^{-1}$ ), while those of the SRVs cover a similar range (median $8.0 \mathrm{~km} \mathrm{~s}^{-1}$, largest value $15.6 \mathrm{~km} \mathrm{~s}^{-1}$ ). Carbon stars have the largest outflow velocities (median $12.0 \mathrm{~km} \mathrm{~s}^{-1}$, largest value $33.2 \mathrm{~km} \mathrm{~s}^{-1}$ ) while as expected $\mathrm{S}$ stars are intermediate (median $8.5 \mathrm{~km} \mathrm{~s}^{-1}$, largest value $24.7 \mathrm{~km} \mathrm{~s}^{-1}$ ). The largest $V_{\mathrm{e}}$ in our sample of $\mathrm{S}$ stars is observed for the CS star TT Cen, a border case between $\mathrm{S}$ and $\mathrm{C}$ stars (see Sect. 2.1). Figure 18 shows that, among Mira S stars, the outflow velocity correlates well with the period of the photometric variations, a result already discussed by Heske (1990) and by Olofsson et al. (1993). Jura (1988) finds an almost identical dependence on period for the ratio of flux densities at 25 and $2.2 \mu \mathrm{m}$.

\subsection{Mass loss rates}

The CO data from Table 6 were used to calculate mass loss rates for the detected $\mathrm{S}$ stars and upper limits for the non-detected stars. The CO lines were modeled using a code based on that of Morris (1980) which assumes spherically-symmetric mass loss at a constant rate and constant outflow speed ${ }^{2}$ with excitation by collisions and by infrared photons at $4.6 \mu \mathrm{m}$. The envelope outer radius was taken to be that at which the $\mathrm{CO}$ is photodestroyed

$2 \quad$ The mass loss rates estimated in the present paper might therefore be inadequate for those stars in Regions D and E with a resolved - possibly detached - shell as observed at $60 \mu \mathrm{m}$.

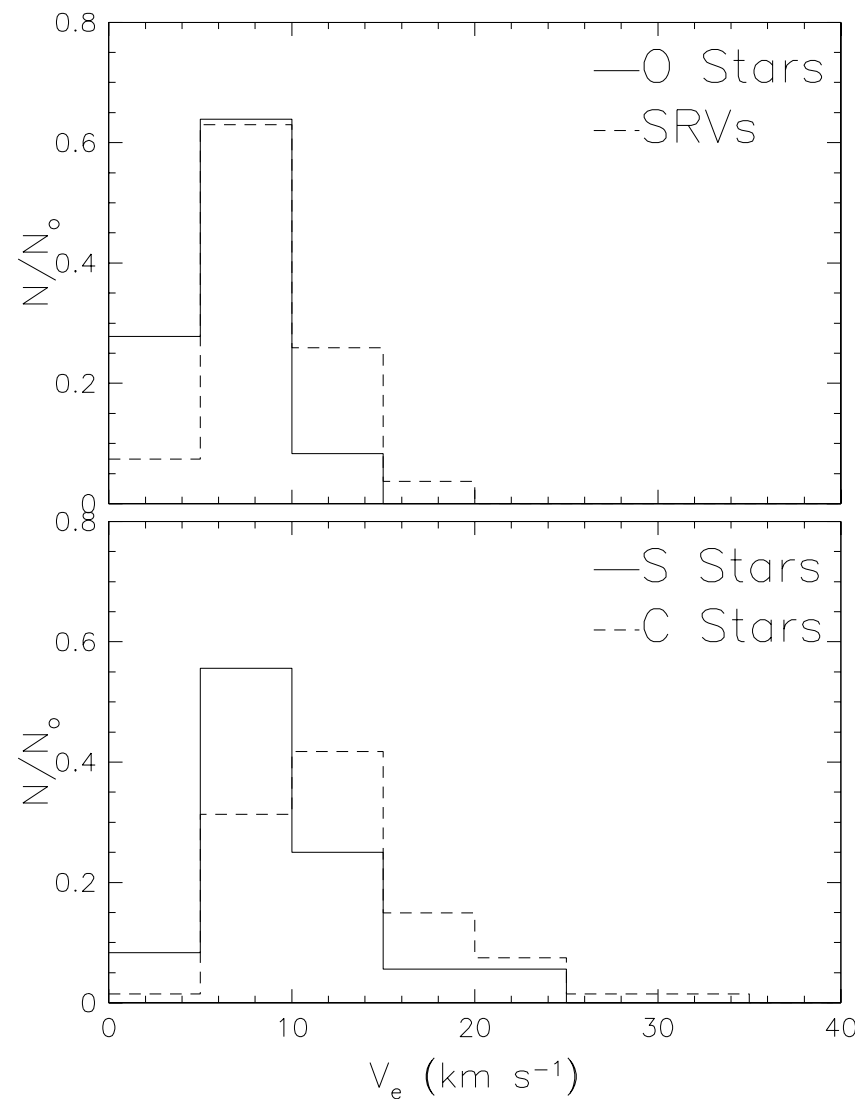

Fig. 17. Normalized histogram of terminal wind outflow speeds of a) oxygen-rich Miras (Young 1995) and semiregular variables (Kerschbaum et al. 1996) and b) S stars (present work) and carbon stars (Olofsson et al. 1993)

by the diffuse interstellar radiation field using the calculations of Mamon et al. (1988). The details are given by Knapp et al. (1997b). The relative abundance of $\mathrm{CO}$ to $\mathrm{H}_{2}$ was assumed to be $6.510^{-4}$ for all stars (Lambert et al. 1986; Smith \& Lambert 1990).

The infrared radiation field was approximated as that of a black body of temperature $2500 \mathrm{~K}$ and radius $2.510^{13}$ $\mathrm{cm}$. Models show that the CO line strength is only weakly dependent on the radiation field, so this simplifying assumption is unlikely to produce an uncertainty of more than $20 \%$ in the derived mass loss rates.

The distances are derived by adopting absolute magnitudes which depend on location in the IR color-color diagram. For stars in Region A (photospheric colors) we assume $M_{\mathrm{bol}}=-2$, i.e. one magnitude below the RGB tip for solar-metallicity stars with $M=1 M_{\odot}$ (Schaller et al. 1992). The bolometric correction $M_{\mathrm{bol}}-M_{\mathrm{K}}$ is derived from the apparent bolometric flux for BD Cam (HR 1105) obtained by integrating the flux densities corresponding to the $U B V R I J K L$ magnitudes from Lee (1970) plus the IRAS flux densities. The corresponding absolute $K$ magnitude is -4.6 . Other authors, e.g. Jura (1988) have used $M_{\mathrm{K}}=-8.1$ for all $\mathrm{S}$ stars; this absolute magnitude is 


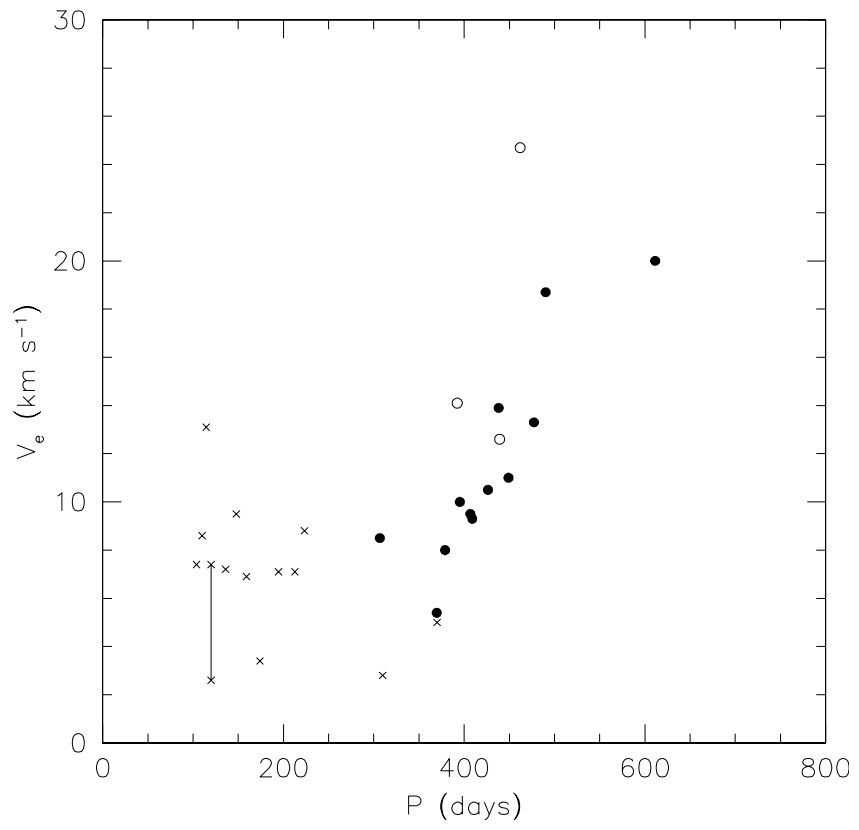

Fig. 18. Terminal wind outflow speed of $S$ stars measured from CO millimeter wavelength emission lines versus period of photometric variations (from the GCVS). Circles: Mira variables (open circles are for stars for which there is only one measurement of $V_{\mathrm{e}}$ ); crosses: semi-regular variables. The points corresponding to the two outflow speeds for RS Cnc are connected by a vertical line. The data for DY Gem, 06331+1414 (SRa; $P=1145 \mathrm{~d}$ ), are not included in the figure

derived from carbon stars in the solar neighborhood and AGB stars in the Magellanic Clouds.

For stars in the other regions, which are supposedly more evolved than are those in Region A, we adopt $M_{\mathrm{K}}=-8.1$. That value of $M_{\mathrm{K}}$ yields distance moduli that are consistent with direct determinations when available. For the composite system $\pi^{1}$ Gru (S5,7e + G0V), Ake \& Johnson (1992) derive a distance modulus of 6.0 from a fit to the UV spectrum, corresponding to a distance of $160 \mathrm{pc}$, identical to the value derived from the $K$ magnitude (Table 1). For T Sgr, a distance of 1000 pc (as compared to $810 \mathrm{pc}$ from the $K$ distance modulus) is derived by Culver \& Ianna (1975) from the spectral type F3IV assigned to its companion. For $\chi$ Cyg, a distance of $136 \mathrm{pc}$ is obtained from the distance modulus in the $K$ band, consistent with that (106 pc) derived from the Hipparcos parallax $(\pi=9.43 \pm 1.36$ mas; van Leeuwen et al. 1997).

The evolutionary status of stars in Regions D and E is unclear, as is their relationship to stars in other regions of the color-color diagram, and so their absolute magnitudes are uncertain. Since these are generally stars of spectral type SC, the choice $M_{\mathrm{K}}=-8.1$ appears to be a reasonable one.
We modeled the wind from RS Cnc as two separate components, fit only to the $\mathrm{CO}(2-1)$ observations described in the previous section.

The upper limits to the mass loss rate of $\mathrm{S}$ stars not detected in $\mathrm{CO}$ were calculated using the median outflow speed of $8.5 \mathrm{~km} \mathrm{~s}^{-1}$ found for the detected sample and assuming that we can detect a line of brightness temperature three times the rms noise (examination of the data in Table 6 suggests that this is reasonable).

The best fit mass loss rates were found by calculating the model peak antenna temperature and integrated line intensities for a given input mass loss rate and comparing it with the observations. The mass loss rates were adjusted until reasonable agreement with all of the observations was found, and were corrected for the mass of helium (see Knapp \& Morris 1985).

The results are listed in the rightmost columns of Table 6 (under the header "Model"), which list the distance, the mean outflow speed (the same for all lines; the value of $8.5 \mathrm{~km} \mathrm{~s}^{-1}$ used for calculating the upper limits is given in parentheses), the $\mathrm{CO}$ photodissociation radius in $\mathrm{cm}$ and in arcseconds, and the predicted $\mathrm{CO}$ peak line temperature and integrated CO line intensity for each of the observed lines. Comparison between the calculations and observations shows that uncertainties in the observations introduce about a factor of two uncertainty into the mass loss rate.

\section{Discussion}

\subsection{Comparison with mass-loss rates of Oxygen and Carbon stars}

Several authors (e.g. Netzer \& Elitzur 1993; Young 1995) have shown that AGB stars with higher mass loss rates have higher outflow velocities. The corresponding relationship for S stars is shown in Fig. 19 and compared with the results for oxygen-rich Miras (Young 1995) and carbon stars (Olofsson et al. 1993). The relationship between $V_{\text {e }}$ and $\dot{M}$ found for oxygen stars by Young (1995) is also shown.

It is not clear at present whether the relationship between $V_{\mathrm{e}}$ and $\dot{M}$ demonstrated by these data is real, in the sense that it has a physical origin, or is due to selection effects. For the present purpose, we will simply treat it as a convenient way to display the data and compare samples. The mass loss rates in Fig. 19 have been calculated assuming a $\mathrm{CO}$ abundance of $f=n(\mathrm{CO}) / n\left(\mathrm{H}_{2}\right)=310^{-4}$ for oxygen stars (Young 1995), $6.510^{-4}$ for S stars and $910^{-4}$ for carbon stars (Olofsson et al. 1993). The S and oxygen stars show essentially the same correspondence between $\dot{M}$ and $V_{\text {e }}$ (though there is a lot more scatter in the S star data), giving some confidence in the assumed value of $f$ and the resulting mass loss rates. The apparently higher values of $V_{\mathrm{e}}$ for a given mass loss rate for carbon stars (see also Fig. 17) will be discussed elsewhere. 


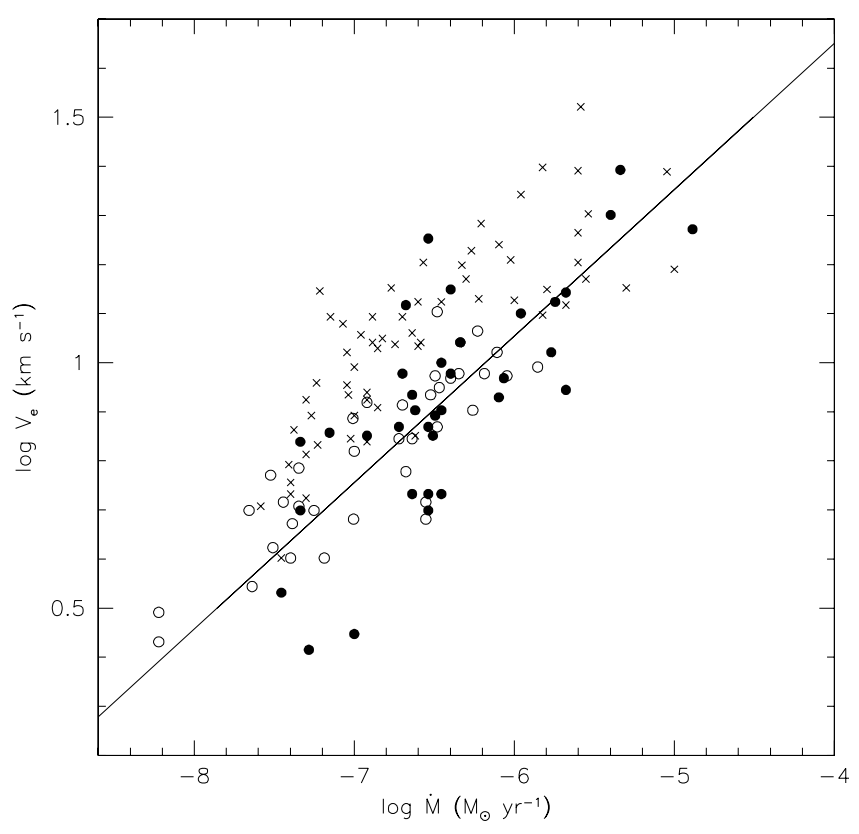

Fig. 19. Wind outflow speed $V_{\mathrm{e}}$ versus mass loss rate for: open circles: oxygen-rich Miras (Young 1995); filled circles: S stars (current work); crosses: carbon stars (Olofsson et al. 1993). The relationship for oxygen Miras found by Young (1995) is also shown

\subsection{Comparison of mass loss rates and color excesses}

The color excess can also be used to give the mass loss rate if the gas-to-dust ratio and the outflow speed are known. This is illustrated in Fig. 20, which shows the envelope density $\dot{M} / V_{\mathrm{e}}$ versus the $12 \mu \mathrm{m} / 2.2 \mu \mathrm{m}$ color. At low mass loss rates $\left(\dot{M} \leq 10^{-7} M_{\odot} \mathrm{y}^{-1}\right)$ the broad-band colors are dominated by the colors of the photosphere, as expected, while the stars with higher mass loss rates show a strong correspondence between the mass loss rate and the $12 \mu \mathrm{m}$ color, showing that the gas to dust ratio in these envelopes is roughly constant. The relationship in Fig. 20 is in agreement with that for oxygen stars shown by Habing (1996).

The two stars TT Cen and RZ Sgr in Region E markedly depart from this relationship, however. These are probably in a transitory phase of evolution; TT Cen is a rare CS star where $\mathrm{ZrO}$ bands seem to have disappeared while $\mathrm{C}_{2}$ bands appeared (see Sect. 2.1 and Stephenson 1973), while RZ Sgr is surrounded by an optical (Whitelock 1994) and IR (YPK) nebula.

Stars with roughly photospheric $12 \mu \mathrm{m} / 2.2 \mu \mathrm{m}$ colors show a wide range in envelope densities. This large scatter may be due to the imperfect coupling between dust and gas at these low densities, which sets a lower limit to the mass loss rate for a radiation-pressure driven wind (cf. Netzer \& Elitzur 1993; SL95). Empirically, this limit is a few $10^{-8} M_{\odot} \mathrm{y}^{-1}$ (Fig. 20 and Table 6 ).

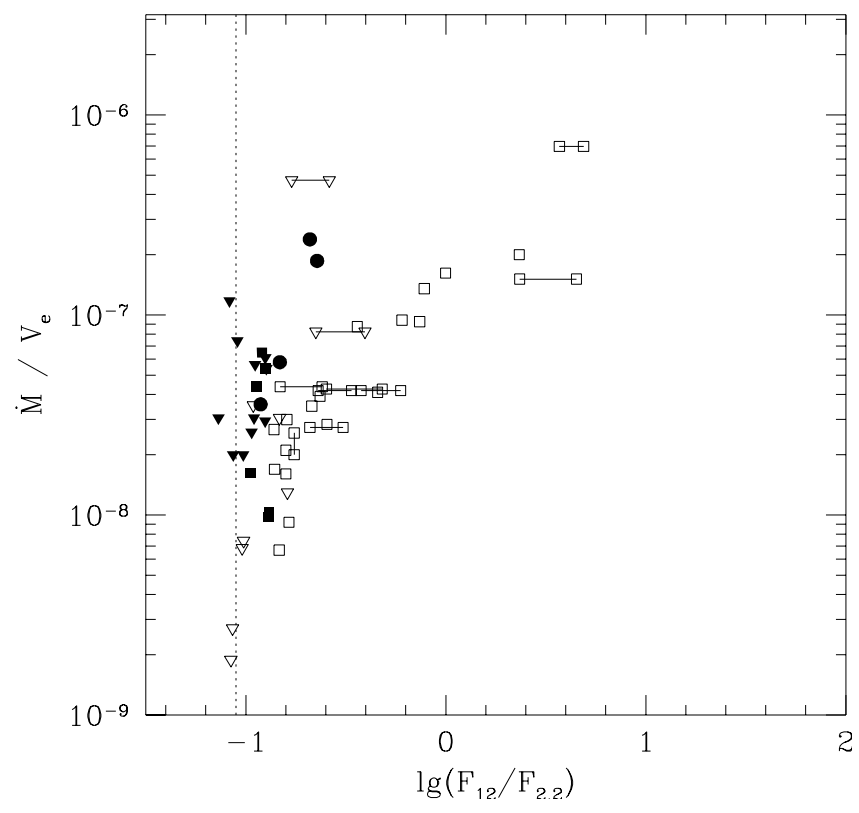

Fig. 20. Envelope density $\dot{M} / V_{\mathrm{e}}$ (measured in $M_{\odot} \mathrm{y}^{-1}$ and $\mathrm{km} \mathrm{s}^{-1}$ ) versus the ratio of $12 \mu \mathrm{m}$ to $2.2 \mu \mathrm{m}$ flux densities. The vertical dotted line shows the photospheric color for a star of temperature $3000 \mathrm{~K}$. Open symbols: stars in Regions A, B and $\mathrm{C}$ of the IR color-color diagram (Fig. 3); filled squares: stars in Region D; filled circles: stars in Region E. The inverted triangles show upper limits

\subsection{Mass loss rates and the IRAS color-color diagram}

Figure 21 presents the mass loss rates of S stars as a function of their location in the $(K-[12],[25]-[60])$ diagram. None of the stars in Region A (stars with photospheric colors) has detectable circumstellar CO emission, with limits on the mass loss rates of $<610^{-8} M_{\odot} \mathrm{y}^{-1}$ and envelope densities well below those of the detected stars (Fig. 20). This confirms the lower limit at which a star can lose mass by a radiation-pressure driven wind estimated by Netzer \& Elitzur (1993). The low mass-loss rates inferred for extrinsic S stars in Region A also confirm an earlier suggestion (Paper I) that these stars are less evolved than the intrinsic S stars populating the other Regions of the IR color-color diagram.

Stars in Regions B and D have moderate mass loss rates, in the range $510^{-8}$ to $510^{-7} M_{\odot} \mathrm{y}^{-1}$. One star from Region B (RS Cnc) has been found to exhibit a double wind (Table 6 ). Most of the observed stars, and most of the detections, lie in Region $\mathrm{C}$, which contains stars with moderately optically thick circumstellar envelopes, likely containing silicate dust. CO emission is detected from 20 of these 23 stars, and the mass loss rates are typically larger than several $\times 10^{-7} M_{\odot} \mathrm{y}^{-1}$. The undetected stars have upper limits greater than this value, so that the data are consistent with the conclusion that all stars in this region lose mass at a rate larger than several $10^{-7} M_{\odot} \mathrm{y}^{-1}$. 


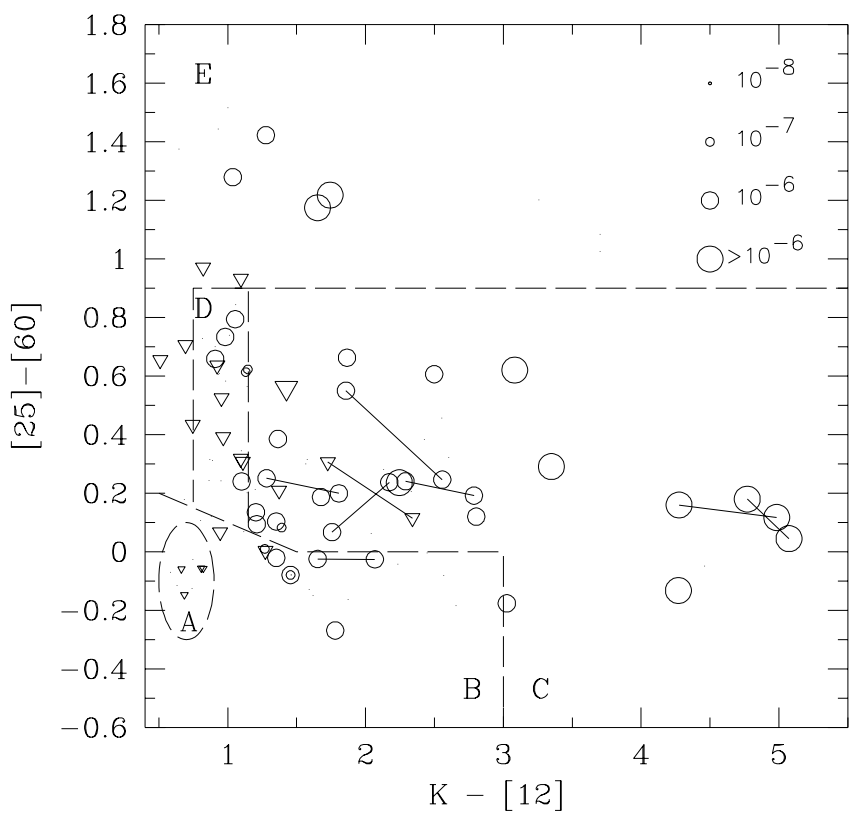

Fig. 21. Mass loss rates of $\mathrm{S}$ stars (as measured from $\mathrm{CO}$ ) in the $(K-[12],[25]-[60])$ diagram. The diameter of the circle is proportional to the mass loss rate, as labeled. Inverted triangles correspond to upper limits

The stars in Region D, which show roughly photospheric $12 \mu \mathrm{m} / 2.2 \mu \mathrm{m}$ colors despite large [25] - [60] indices, generally have low mass-loss rates (like Region B). Stars in Region E have mixed properties. Some, like TT Cen and RZ Sgr, lose mass at a very large rate (several $\left.10^{-6} M_{\odot} \mathrm{y}^{-1}\right)$, while others like FU Mon lose mass at a more moderate rate (a few $10^{-7} M_{\odot} \mathrm{y}^{-1}$ ). Figure 22 presents the variation of the wind velocity across the $(K-[12],[25]-[60])$ diagram, and shows that stars in Region $\mathrm{E}$ also have a wide range of wind velocities, from very low (FU Mon: $2.8 \mathrm{~km} \mathrm{~s}^{-1}$ ) to very large (TT Cen: $\left.24.7 \mathrm{~km} \mathrm{~s}^{-1}\right)$.

FU Mon also has a resolved IR envelope (Sect. 2.4), and this fact taken together with a low mass loss rate and a small outflow velocity suggest that mass loss has just resumed in that star. Olofsson et al. $(1990 ; 1993)$ detected several carbon stars (S Sct, U Ant, TT Cyg) with a detached shell and a double wind that would also fall in our Region E. The older, detached shell was produced by a massive, fast wind, whereas the recent shell is caused by a slow ( $5 \mathrm{~km} \mathrm{~s}^{-1}$ ), low mass loss rate wind. Since FU Mon is an SC star with a C/O ratio close to unity (Dominy et al. 1986), the mass loss may have come to a halt when the the $\mathrm{C} / \mathrm{O}$ ratio approached unity, as already suggested by Willems \& de Jong (1988) and Chan \& Kwok (1988). This scenario may well hold for all SC stars, even though some, like UY Cen, are in fact located in Region C. As argued in Sect. 4.2, that star may be at the end of the loop in the IR color-color diagram. The horn-shaped CO line profile of UY Cen observed by SL95, indicative of a detached

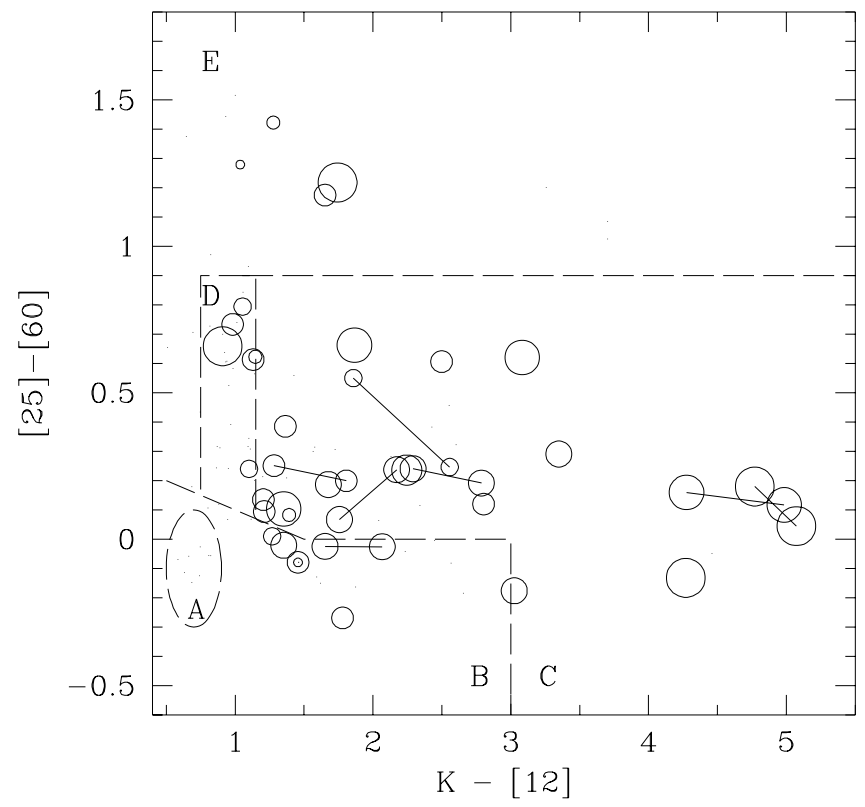

Fig. 22. The outflow velocity as measured from $\mathrm{CO}$ for $\mathrm{S}$ stars in the $(K-[12],[25]-[60])$ diagram. The diameter of the circle is proportional to the outflow velocity. Compare to Fig. 10 of Olofsson et al. (1993) for C stars

shell and fossil mass loss, supports this idea. Finally, other stars lying in Region E like TT Cen, RZ Sgr and DK Vul have peculiar CO line profiles (narrow central feature superimposed on a broader less intense feature) suggesting that they have multiple winds.

\subsection{Mass loss rates and binaries}

Several intrinsic S stars in the sample considered in this paper are binaries with main sequence companions, as revealed by the composite nature of their spectrum at minimum light (WY Cas, W Aql, T Sgr; Herbig 1965; Culver \& Ianna 1975). A composite spectrum is also suspected for S Lyr from its shallow lightcurve (Merrill 1956). The star $\pi^{1}$ Gru has a close G0V visual companion (Feast 1953). W Aql, WY Cas and S Lyr are surely among the S stars with the largest mass loss rates, while T Sgr and $\pi^{1}$ Gru have mass loss rates close to the average for stars in Region C. Clearly, a definite conclusion as to whether binarity can indeed reinforce the mass loss rate, e.g. by the companionreinforced attrition process (Eggleton 1986), would require the knowledge of the orbital separation of these systems.

It has sometimes been argued (e.g. Eggleton 1986; Tout \& Eggleton 1988; Kenyon 1994; Han et al. 1995) that the mass loss rates of giant stars in binary systems must be larger than those of single red giants. More precisely, Han et al. (1995) suggest that the mass loss rate of a giant approaching its Roche lobe in a binary system exceeds by more than a factor $10^{3}$ the rate predicted by the Reimers formula (Reimers 1975). As far as the binary, extrinsic S 
stars are concerned, this effect, if present, is clearly not large enough to bring their mass loss rates to the level of the intrinsic S stars. The difference in the evolutionary stages of extrinsic and intrinsic $\mathrm{S}$ stars, believed to be RGB (or Early-AGB, according to the terminology of Iben \& Renzini 1983) and TP-AGB stars, respectively, thus appears to be of greater importance for the mass loss rate than their binary or non-binary character.

\section{Summary}

Our extensive discussion of the circumstellar properties of $\mathrm{S}$ stars confirms the dichotomy extrinsic/intrinsic S stars and leads to the following conclusions.

Extrinsic S stars have the lowest mass loss rates among S stars $\left(<210^{-8} M_{\odot} \mathrm{y}^{-1}\right)$, and undetectable circumstellar shells. This is consistent with the hypothesis that these stars are much less evolved than intrinsic $\mathrm{S}$ stars, populating the RGB or Early-AGB rather than the TP-AGB like intrinsic $\mathrm{S}$ stars. The binary character of these stars does not seem to increase their mass loss rate, as it has sometimes been suggested, e.g., in discussions relating to symbiotic or barium stars. Note, however, that the few binary intrinsic $\mathrm{S}$ stars are among the stars with the largest mass loss rates in our sample.

Among intrinsic $\mathrm{S}$ stars, various subclasses must be distinguished:

1. A few Tc-rich S stars (e.g., HR Peg) have very low mass-loss rates, similar to those of the extrinsic S stars, with undetectable circumstellar shells [Region A of the $(K-[12],[25]-[60])$ color-color diagram]. These are $\mathrm{S}$ stars with weak chemical peculiarities, barely distinguishable from normal $\mathrm{M}$ giants.

2. S stars with weak chemical peculiarities and tenuous O-rich circumstellar shells (small $K-[12]$ excess), fed by a small albeit measurable mass loss rate of a few $10^{-7} M_{\odot} \mathrm{y}^{-1}$ (Region B). These are short-period $(P \sim$ 100 to $150 \mathrm{~d})$ SR variables or short-period $(P<500 \mathrm{~d})$ Mira variables. This region may be contaminated by (possibly normal $\mathrm{M}$ ) supergiants of variability type SRc (T Cet, RS Cnc, Y Lyn). These SRc supergiants have resolved shells at $60 \mu \mathrm{m}$, and for RS Cnc, a double wind.

3. S stars with strong chemical peculiarities and dense Orich circumstellar shells (as indicated by $\mathrm{SiO}$ maser emission, the $9.7 \mu \mathrm{m}$ silicate feature and large $K-[12]$ indices), populating the lower part of Region C. Mass loss rates range from several $10^{-7}$ to $10^{-5} M_{\odot} \mathrm{y}^{-1}$. Almost all of them are long-period $(P>300 \mathrm{~d})$ Mira variables.

4. S stars with strong chemical peculiarities (often classified as $\mathrm{SC}$ in the optical), with neither $\mathrm{SiO}$ maser emission nor $9.7 \mu \mathrm{m}$ silicate emission, with featureless IRAS spectra and moderate to large [25] - [60] indices. These stars populate Region D and the upper part of Region C (like optical carbon stars) and have moderate mass loss rates (a few $10^{-8}$ to a few $10^{-7} M_{\odot} \mathrm{y}^{-1}$ ) with small wind veloci- ties (generally $\lesssim 8 \mathrm{~km} \mathrm{~s}^{-1}$ ). They are mainly semi-regular (with periods ranging from 60 to $360 \mathrm{~d}$ ) or irregular variables, with a few short-period $(P<370 \mathrm{~d})$ Mira variables. These properties are reminiscent of carbon stars. All their features may equally well be explained by a carbon-rich circumstellar shell, or by a detached shell. The relationship between stars in Regions C and D is far from being clear. It may be a difference of galactic populations, as suggested by their different properties as variable stars, or stars might oscillate between Regions $\mathrm{C}$ and $\mathrm{D}$ at different phases of their variability cycle, as observed for some variable IRAS sources in our sample, or they may be on different parts of the loop associated with the detachment of a dust shell.

5. A few S stars have well-resolved (and thus very extended) IR shells (also visible in the optical in the case of RZ Sgr), probably detached from their parent star, as indicated by strong 60 and $100 \mu \mathrm{m}$ excesses (Region E). The mass loss rates span a wide range, from a few $10^{-7}$ to several $10^{-6} M_{\odot} \mathrm{y}^{-1}$, as do the wind velocities $\left(25 \mathrm{~km} \mathrm{~s}^{-1}\right.$ for TT Cen to $2.8 \mathrm{~km} \mathrm{~s}^{-1}$ for FU Mon). These stars with extreme properties are often SC or CS stars, and may be experiencing a loop in the IRAS color-color diagram as first proposed by Willems \& de Jong (1988), triggered by their $\mathrm{C} / \mathrm{O}$ ratio being very close to unity. In TT Cen, the dominant spectral features are $\mathrm{ZrO}$ bands at some times, and $\mathrm{C}_{2}$ bands at other times. This is a rare and short-lived phase, and thus does not face the difficulties generally opposed to the Willems \& de Jong scenario. Some SC or CS stars in Region C, like BH Cru (a sister case of TT Cen) and UY Cen, may be at the end of the counter-clockwise loop in the IRAS color-color diagram, entering the region of heavily-obscured IR carbon stars. Region $\mathrm{E}$ is probably fed by stars leaving Region $\mathrm{C}$ when their $\mathrm{C} / \mathrm{O}$ ratio reaches values close to unity. However, there is no need that all S stars experience such a loop.

Acknowledgements. We thank the staff at CSO, especially Ken Young (Taco), Antony Schinkel, Maren Purves and Tom Phillips, for the observing time and for their help with the observations. Astronomical research at the CSO is supported by the National Science Foundation via grants AST96-15025. We thank the staff at IPAC for swiftly processing the numerous requests for IRAS archive data sent over the Internet. We especially thank Ron Beck for providing us with the IRAS template data. We thank Princeton University, the Fonds National de la Recherche Scientifique (Belgium) and the National Science Foundation (U.S.A.), via grant AST96-18503, for partial support of this work. The CO line formation modeling was based on code by Mark Morris, and the figures were drawn using software by Robert Lupton and Patricia Monger. This research has made use of the Simbad data base, operated at CDS, Strasbourg, France. A.J. is Research Associate, F.N.R.S. (Belgium). 
Table A1. IRAS flux densities for $\chi$ Cyg

\begin{tabular}{crrrrrrrr} 
JD - 2440 000 & $\phi$ & $\begin{array}{r}F 12 \\
(\mathrm{Jy})\end{array}$ & $\begin{array}{r}\text { offset } \\
\left({ }^{\prime}\right)\end{array}$ & det & $\begin{array}{r}F 25 \\
(\mathrm{Jy})\end{array}$ & $\begin{array}{r}F 60 \\
(\mathrm{Jy})\end{array}$ & {$[12]-[25]$} & {$[25]-[60]$} \\
\hline \multirow{2}{*}{5452.8} & 0.12 & 1860.6 & 0.40 & 48 & 643.5 & 99.4 & 0.41 & -0.15 \\
& & & & & 540.2 & & 0.22 & 0.04 \\
5453.7 & 0.12 & 1778.6 & -1.01 & 23 & 533.2 & 97.7 & 0.25 & 0.04 \\
& & 1880.7 & 1.23 & 51 & & 92.6 & 0.19 & -0.02 \\
5466.1 & 0.15 & 1157.5 & -1.79 & 28 & 626.6 & 86.5 & 0.89 & -0.27 \\
& & 1975.0 & 0.63 & 48 & 525.0 & 99.8 & 0.37 & -0.11 \\
& & & & & & & 0.70 & -0.08 \\
5466.2 & 0.15 & 1624.5 & -0.03 & 53 & 520.7 & 98.7 & 0.32 & 0.08 \\
& & & & & 583.0 & & 0.45 & -0.05 \\
5639.7 & 0.58 & 1248.7 & -0.22 & 30 & 456.3 & 72.6 & 0.47 & -0.11 \\
& & & & & 422.1 & & 0.38 & -0.03 \\
5639.8 & 0.58 & 1172.5 & -0.52 & 24 & 433.5 & 69.5 & 0.48 & -0.11 \\
& & 1266.5 & 1.72 & 49 & & & 0.40 & \\
5640.3 & 0.58 & 1184.6 & -0.04 & 24 & 404.9 & 70.8 & 0.39 & -0.01 \\
5640.6 & 0.58 & 1249.0 & 0.13 & 27 & 383.0 & 74.7 & 0.28 & 0.11 \\
& & 1214.4 & 1.75 & 51 & & & 0.31 & \\
\hline
\end{tabular}

\section{Appendix A: Stellar variability and the IRAS flux densities}

As discussed in Sect. 2.2, the flux densities obtained from co-addition of the IRAS data generally agree well with those in the PSC with, however, some notable exceptions, as shown in Fig. 1. Several stars have flux densities which are in disagreement at a level well outside that expected from noise. In this Appendix we investigate whether these disagreements can be explained by stellar variability, using the data for $\chi$ Cyg as an example. The ephemerides listed in the GCVS give a variation of about 6 mag at $V$ between maximum and minimum light, a period of $408 \mathrm{~d}$ and the epoch of zero phase which is closest to the IRAS launch date of JD 2445404.4.

The colors of $\chi \mathrm{Cyg}$, calculated from the IRAS PSC flux densities (see Table A2), are photospheric, despite the strong circumstellar CO emission (discussed in Sect. 5). On the other hand, model IRAS colors calculated from the mass loss rate given by the $\mathrm{CO}$ lines (several $10^{-7} M_{\odot} \mathrm{y}^{-1}$ ), assuming a normal gas to dust ratio and silicate grains (the star is an $\mathrm{SiO}$ maser; e.g. Patel et al. 1992), are well displaced from photospheric values. This discrepancy does not mean that the circumstellar envelope is dust-free, however; the ratio of the $12 \mu \mathrm{m}$ to $2 \mu \mathrm{m}$ flux densities is well in excess of the photospheric value for a temperature of $2400 \mathrm{~K}$ (Haniff et al. 1995), demonstrating the presence of an appropriate amount of circumstellar dust.

We therefore re-examined the raw IRAS data for this star. The individual IRAS $12 \mu \mathrm{m}, 25 \mu \mathrm{m}$, and $60 \mu \mathrm{m}$ observations for $\chi$ Cyg are listed in Table A1. Column 1 gives the Julian date of the observations computed from the "Satellite Operation Plan" number attached to each scan and from the mission chronology provided by the Explanatory Supplement. Column 2 lists the phase. Next are the flux density, the offset between the scan center and the stellar position, and the detector number for the $12 \mu \mathrm{m}$ data. The final columns contain the $25 \mu \mathrm{m}$ and $60 \mu \mathrm{m}$ flux densities and the [12] - [25] and [25] - [60] colors calculated from combinations of the observed flux densities. The colors calculated from the flux densities derived from individual scans locate $\chi \mathrm{Cyg}$ in Region $\mathrm{B}$ of the color-color diagram.

Table A1 shows that there are occasionally large discrepancies between the flux densities measured by different detectors at the same time; see, for example, the $12 \mu \mathrm{m}$ flux densities observed at JD 2445466.1. The low flux density is probably due to the large offset between the stellar and detector center positions; such discrepant observations are usually filtered out in the IRAS data processing. Real variability is also apparent. The data were obtained at two epochs, near maximum and minimum phase, and the flux densities at all four wavelengths are systematically higher for the first set of observations than the second. The mean flux densities for these two epochs are listed in Table A2; the differences are $\Delta=$ $\left(F_{\max }-F_{\min }\right) / F_{\max }=32 \%$ at $12 \mu \mathrm{m}, 26 \%$ at $25 \mu \mathrm{m}, 25 \%$ at $60 \mu \mathrm{m}$ and $31 \%$ at $100 \mu \mathrm{m}$.

Can these variations be attributed to the stellar variability? The huge variations in Mira variables at visible wavelengths are due largely to the changing photospheric temperature; the variation in the bolometric magnitude 
Table A2. Observed and model IRAS flux densities for $\chi$ Cyg. The PSC fluxes are also listed

\begin{tabular}{lllll} 
Phase & $F 12$ & $F 25$ & $F 60$ & $F 100$ \\
& $(\mathrm{Jy})$ & $(\mathrm{Jy})$ & $(\mathrm{Jy})$ & $(\mathrm{Jy})$ \\
\hline max & 1804 & 568 & 96 & 20 \\
$\min$ & 1223 & 420 & 72 & 14 \\
PSC & 1688 & 459 & 81 & 18 \\
\hline \multicolumn{5}{c}{ Calculated } \\
Phase & $F 12$ & $F 25$ & $F 60$ & $F 100$ \\
& $(\mathrm{Jy})$ & $(\mathrm{Jy})$ & $(\mathrm{Jy})$ & $(\mathrm{Jy})$ \\
\hline \multicolumn{5}{c}{} \\
max & 1486 & 576 & 86 & 20 \\
min & 1108 & 486 & 79 & 17 \\
\hline
\end{tabular}

is much smaller, about a factor of 2 (e.g. Petit 1982; Hoffmeister et al. 1985). Because of the variation in stellar effective temperature, the star reaches maximum light later at longer wavelengths in the visible and near infrared (e.g. Le Bertre 1992). In particular, the variation at visible wavelengths leads the total light variation by about 0.1 of a period. The two groups of observations in Table A1 were thus made close to maximum and minimum luminosity.

We modeled the object as a star with a circumstellar envelope and varied the luminosity of the model star. The envelope contains silicate grains, has a dust loss rate of $810^{-10} M_{\odot} \mathrm{y}^{-1}$ and is assumed to have the same outflow speed as the gas $\left(9.5 \mathrm{~km} \mathrm{~s}^{-1}\right)$. The star is assumed to be a black body of temperature $2400 \mathrm{~K}$ and luminosity $3000 L_{\odot}$ at minimum, and $2800 \mathrm{~K}$ and $6000 L_{\odot}$ at maximum. The resulting model flux densities are listed in Table A2 and are reasonably close to the observed values (note that the discrepancy at $12 \mu \mathrm{m}$ may be caused in part by the saturation of the detectors at these flux densities exceeding $1000 \mathrm{Jy})$. The variation in the IRAS flux densities can thus be fully explained by the stellar variability.

These results show that caution is required in the interpretation of IRAS data for variable stars; colors must be calculated from data taken at the same epoch, as should models of the circumstellar envelope. Variability introduces a significant amount of scatter into the IRAS colors, especially [12] - [25], which as a result does not provide the clean measure of the stellar mass loss rate which the models predict (see, for example, the discussion by Habing 1996).

\section{References}

Ake T.B., Johnson H.R., 1992, BAAS 24, 788

Beckwith S., Evans N.J., Becklin E.E., Neugebauer G., 1976, ApJ 208, 390

Benson P.J., Little-Marenin I.R., Woods T.C., et al., 1990, ApJS 74, 911

Bessell M.S., Wood P.R., Lloyd Evans T., 1983, MNRAS 202, 59

Bieging J.H., Latter W.B., 1994, ApJ 422, 765 (BL94)
Brown J.A., Smith V.V., Lambert D.L., et al., 1990, AJ 99, 1930

Catchpole R.M., Robertson B.S.C., Lloyd Evans T.H.H., et al., 1979, SAAO Circ. 1, 61

Chan S.J., Kwok S., 1988, ApJ 334, 362

Chen P.S., Gao H., Chen Y.K., Dong H.W., 1988, A\&AS 72, 239

Chen P.S., Kwok S., 1993, ApJ 416, 769

Chen P.S., Gao H., Jorissen A., 1995, A\&AS 113, 51

Culver R.B., Ianna P.A., 1975, ApJ 195, L37

Deguchi S., Nakada Y., Forster J.R., 1989, MNRAS 239, 825

de Jong T., 1989, A\&A 223, L23

Dominy J.F., Wallerstein G., Suntzeff N.B., 1986, ApJ 300, 325

Draine B.T., 1985, ApJS 57, 587

Draine B.T., Lee H.M., 1984, ApJ 285, 89

Epchtein N., Le Bertre T., Lépine J.R.D., et al., 1987, A\&AS 71,39

Epchtein N., Le Bertre T., Lépine J.R.D., 1990, A\&A 227, 82

Eggleton P.P., 1986. In: Trümper J., Lewin W.H.G., Brinkmann W. (eds.). The Evolution of Galactic X-ray Binaries. Reidel, Dordrecht, p. 87

Feast M.W., 1953, MNRAS 113, 510

Frost C.A., Lattanzio J.C., 1995. In: Noels A., Fraipont D., Gabriel M., Grevesse N., Demarque P. (eds.) Stellar Evolution: What Should Be Done? (Proc. 32nd Liège Intern. Astrophys. Coll.). Univ. de Liège, p. 307

Groenewegen M.A.T., 1993, A\&A 271, 180

Guglielmo F., Epchtein N., Le Bertre T., et al., 1993, A\&AS 99,31

Habing H.J., 1996, A\&AR 7, 97

Hacking P., Neugebauer G., Emerson J., et al., 1985, PASP 97, 616

Han Z., Eggleton P.P., Podsiadlowski P., Tout C.A., 1995, MNRAS 277, 1443

Haniff C.A., Scholz M., Tuthill P.G., 1995, MNRAS 276, 640

Herbig G.H., 1965, Kleine Veroff. Remeis-Sternwarte 4, 40, 164

Herwig F., Blöcker T., Schönberner D., El Eid M., 1997, A\&A $324, \mathrm{~L} 81$

Heske A., 1990, A\&A 229, 494

Hoffmeister C., Richter G., Wenzel W., 1985, Veränderliche Sterne. Springer-Verlag

Hoffleit D., 1982, The Bright Star Catalogue (4th ed.), Yale University Observatory, New Haven

Houk N., Cowley A.P., 1975, Michigan Catalogue of TwoDimensional Spectral Types for the HD stars. Univ. Michigan, Ann Arbor, Vol. 1

Iben I. Jr., Renzini A., 1983, ARA\&A 21, 271

IRAS Explanatory Supplement, Second Edition, 1988, Joint IRAS Science Working Group, Washington DC, US GPO

IRAS Point Source Catalogue, Second Edition, 1988, Joint IRAS Science Working Group, Washington DC, US GPO

IRAS Small-Scale Structure Catalogue, 1985, Joint IRAS Science Working Group, Washington DC, US GPO (SSSC)

Ivezić Z., Elitzur M., 1995, ApJ 445, 415

Johnson H.R., Ake T.B., Ameen M.M., 1993, ApJ 402, 667

Jorissen A., Mayor M., 1992, A\&A 260, 115

Jorissen A., Frayer D.T., Johnson H.R., Mayor M., Smith V.V., 1993, A\&A 271, 463 (Paper I)

Jorissen A., Van Eck S., Mayor M., Udry S., 1998, A\&A (in press)

Jura M., 1988, ApJS 66, 33 
Kahane C., Jura M., 1994, A\&A 290, 183

Kahane C., Jura M., 1996, A\&A 310, 952

Käppeler F., Beer H., Wisshak K., 1989, Rep. Prog. Phys. 52, 945

Keenan P.C., 1954, ApJ 120, 484

Keenan P.C., Boeshaar P.C., 1980, ApJS 43, 379

Keenan P.C., McNeil R.C., 1989, ApJS 71, 245

Kenyon S.J., 1994, Mem. Soc. Astron. It. 65, 135

Kerschbaum F., Olofsson H., Hron J., 1996, A\&A 311, 273

Kholopov P.N., Samus' N.N., Frolov M.S., et al., 1985, General Catalogue of Variable Stars (4th ed.), Moscow, Nauka (GCVS)

Knapp G.R., Morris M., 1985, ApJ 292, 640

Knapp G.R., Young K., Lee E., Jorissen A., 1997a, ApJ (submitted)

Knapp G.R., Woodhams M.D., Gammie C.F., Young K., Phillips T.G., $1997 \mathrm{~b}$ (in preparation)

Kolena J., Pataki L., 1977, AJ 82, 150

Lambert D.L., Gustafsson B., Eriksson K., Hinkle K.H., 1986, ApJS 62, 373

Lambert D.L., Smith V.V., Busso M., Gallino R., Straniero O., 1995, ApJ 450, 302

Le Bertre T., 1992, A\&AS 94, 377

Lee T.A., 1970, ApJ 162, 217

Levine D.A., et al., 1993, Infrared Processing and Analysis Center User's Guide (edition 5), MS 100-22, CalTech, Pasadena

Lewis B.M., 1989, AJ 98, 1814

Lewis B.M., 1996, ApJ 462, 786

Little S.J., Little-Marenin I.R., Hagen-Bauer W., 1987, AJ 94, 981

Lloyd Evans T., 1983, MNRAS 204, 985

Lloyd Evans T., 1984, MNRAS 208, 447

Lloyd Evans T., 1985, in: Jaschek M., Keenan P.C. (eds.) Cool Stars with Excesses of Heavy Elements. Reidel, Dordrecht, p. 163

Lloyd Evans T., Catchpole R.M., 1989, MNRAS 237, 219

Loup C., Forveille T., Omont A., Paul J.F., 1993, A\&A 99, 291

Mamon G. A., Glassgold A.E., Huggins P.J., 1988, ApJ 328, 797

Margulis M., van Blerkom D.J., Snell R.L., Kleinmann S.G., 1990, ApJ 361, 763

Mathews G.J., Takahashi K., Ward R.A., Howard W.M., 1986, ApJ 302, 410

McClure R.D., 1983, ApJ 268, 264

McClure R.D., Fletcher J.M., Nemec J.M., 1980, ApJ 238, L35

Meadows P.J., Good A.R., Wolstencroft R.D., 1987, MNRAS $225,43 \mathrm{P}$

Merrill P.W., 1922, ApJ 56, 457

Merrill P.W., 1952, ApJ 116, 21

Merrill P.W., 1956, PASP 68, 162

Mendoza V.E., Johnson H.L., 1965, ApJ 141, 161

Mihalas D., Binney J., 1981. Galactic astronomy (2nd ed.), W.H. Freeman \& Co., San Francisco

Morris M., 1980, ApJ 236, 823

Netzer N., Elitzur M., 1993, ApJ 410, 701

Neugebauer G., Leighton R.B., 1969, Two-Micron Sky Survey, NASA SP-3047 (TMSS)

Noguchi K., Sun J., Wang G., 1991, PASJ 43, 311

Nyman L.-Å, Booth R.S., Carlström U., et al., 1992, A\&AS 93,121
Nyman L.-Å, Olofsson H., Johansson L.E.B., et al., 1993, A\&A 269, 377

Olnon F.M., Raimond E., Neugebauer G., Van Duinen R.J., Habing H.J., 1986, A\&AS 65, 607

Olofsson H., Carlström U., Eriksson K., Gustafsson B., Willson L.A., 1990, A\&A 230, L13

Olofsson H., Eriksson K., Gustafsson B., Carlström U., 1993, ApJS 87, 267

Omont A., Loup C., Forveille T., et al., 1993, A\&A 267, 515

Patel N.A., Joseph A., Ganesan R., 1992, J. Astrophys. Astron. 13,241

Petit M., 1982, Variable Stars. J. Wiley \& Son

Piccirillo J., 1980, MNRAS 190, 441

Price S.D., 1968, AJ 73, 431

Reimers D., 1975, Mem. Soc. Roy. Sci. Liège, 6th Ser., 8, 369

Rouleau F., Martin P.G., 1991, ApJ 377, 526

Rudnitskij G.M., 1976, Soviet AJ 20, 693

Sackmann I.-J., Boothroyd A.I., 1991. In: Evolution of Stars: The Photospheric Abundance Connection, Michaud G. \& Tutukov A. (eds.), IAU Symp. 145. Kluwer, Dordrecht, p. 275

Sahai R., 1992, A\&A 253, L33

Sahai R., Liechti S., 1995, A\&A 293, 198 (SL95)

Scalo J.M., Ross J.E., 1976, A\&A 48, 219

Schaller G., Schaerer G., Meynet G., Maeder A., 1992, A\&AS 96, 269

Smith V.V., Lambert D.L., 1988, ApJ 333, 219

Smith V.V., Lambert D.L., 1990, ApJS 72, 387

Stanek K.Z., Knapp G.R., Young K., Phillips T.G., 1995, ApJS 100, 169

Stephenson C.B., 1973, ApJ 186, 589

Stephenson C.B., 1984, Publ. Warner \& Swasey Observ., Vol. 3, No. 1 (GCGSS)

Stephenson C.B., 1989, Publ. Warner \& Swasey Observ., Vol. 3, No. 2

Stephenson C.B., 1990, AJ 100, 569

Tout C.A., Eggleton P.P., 1988, MNRAS 231, 823

Udry S., Jorissen A., Mayor M., Van Eck S., 1998, A\&A (in press)

van der Veen W.E.C.J., Habing H.J., 1988, A\&A 194, 125 (VH)

Van Eck S., Jorissen A., Mayor M., Udry S., Burnet M., 1997, in: Wing R. (ed.) The Carbon Star Phenomenon, IAU Symp. 177. Kluwer, Dordrecht (in press)

van Leeuwen F., Feast M.W., Whitelock P.A., Yudin B., 1997, MNRAS 287, 955

Volk K., Cohen M., 1989, AJ 98, 931

Wallerstein G., Dominy J.F., 1988, ApJ 326, 292

Westerlund B.E., Olander N., 1978, A\&AS 32, 401

Whitelock P., 1994, MNRAS 270, L15

Willems F.J., de Jong T., 1986, ApJ 309, L39

Willems F.J., de Jong T., 1988, A\&A 196, 173

Wilson R.E., 1953, A General Catalogue of Stellar Radial Velocities. Carnegie Inst. Washington Publ., p. 601

Winfrey S., Barnbaum C., Morris M., Omont A., 1994, BAAS 26, 1382

Young K., 1995, ApJ 445, 872

Young K., Phillips T.G., Knapp G.R., 1993a, ApJS 86, 517 (YPK)

Young K., Phillips T.G., Knapp G.R., 1993b, ApJ 409, 725

Zuckerman B., Maddalena R.J., 1989, A\&A 223, L20 
Table 1. IRAS co-added flux densities for S stars, grouped according to their location in the $(K-[12]$, [25] - [60]) diagram

Region A: Stellar photospheres

\begin{tabular}{|c|c|c|c|c|c|c|c|c|c|c|c|c|c|c|}
\hline GCGSS IRAS & $\begin{array}{r}F 2.2 \\
(\mathrm{Jy}) \\
\end{array}$ & & $\begin{array}{l}F 12 \\
(\mathrm{Jy}) \\
\end{array}$ & $\begin{array}{l}F 25 \\
(\mathrm{Jy})\end{array}$ & $\begin{array}{l}F 60 \\
(\mathrm{Jy})\end{array}$ & $\begin{array}{r}F 100 \\
(\mathrm{Jy}) \\
\end{array}$ & Tc & LRS & VC & Sp & Var & $\begin{array}{l}P \\
\text { (d) }\end{array}$ & $\begin{array}{l}\Delta V \\
(\mathrm{mag})\end{array}$ & Name \\
\hline $2601113+2815$ & 139.4 & 2 & 11.95 & 3.02 & 0.63 & & no & & $\mathrm{F}$ & $\mathrm{S} 3 / 2$ & & & & HR 363 \\
\hline $7903377+6303$ & 502.0 & 2 & 42.23 & 10.86 & 1.82 & & no & 18 & & $\mathrm{~S} 4 / 2$ & $\mathrm{Lb}$ & & 0.1 & BD Cam, HR \\
\hline 133 05199-0842 & 92.1 & 2 & 7.94 & 2.06 & & & no & 16 & $\mathrm{~S}$ & $\mathrm{~S} 4,1$ & & & & HD 35155 \\
\hline $38207392+1419$ & 331.4 & 2 & 25.91 & 6.51 & 1.08 & 0.37 & & 18 & & M3S & SR & & 0.2 & NZ Gem, HD 61913 \\
\hline $72911098-3209$ & 112.8 & 2 & 9.21 & 2.45 & 0.39 & & & & $\mathrm{~S}$ & Swk & & & 0.1 & NSV 5129, HR 4346 \\
\hline $79612272-4127$ & 207.2 & 1 & 17.77 & 4.47 & 0.69 & & & 18 & $\mathrm{~S}$ & M3-IIIa & & & & NSV 5655, HR 4755 \\
\hline $80413079-8931$ & 167.6 & 1 & 15.23 & 3.88 & 0.67 & & & 17 & $\mathrm{~S}$ & $\mathrm{~S} 5,1$ & Lb: & & & BQ Oct, HD 110994 \\
\hline $826 \quad 13372-7136$ & 251.4 & 1 & 24.13 & 6.43 & 1.08 & & & 18 & $\mathrm{~S}$ & $\mathrm{~S} 6,2$ & & & & HD 118685 \\
\hline 938 16425-1902 & 150.1 & 2 & 12.24 & 3.06 & $a$ & & no & 31 & $\mathrm{~S}$ & Swk & & & & HD 151011 \\
\hline $131522521+1640$ & 283.4 & 2 & 27.58 & 7.33 & 1.23 & 0.67 & yes & & $\mathrm{S}$ & $\mathrm{S} 4 / 1$ & $\mathrm{SRb}$ & 50 & 0.3 & HR Peg, HR 8714 \\
\hline $132223070+0824$ & 938.4 & 2 & 84.67 & 20.31 & 3.20 & 0.96 & no & 18 & & M4S & $\mathrm{SRa}$ & 93 & 0.3 & GZ Peg, 57 Peg \\
\hline
\end{tabular}

a: Strong cirrus contamination.

Region B: Small ZrO index and no [25] - [60] excess

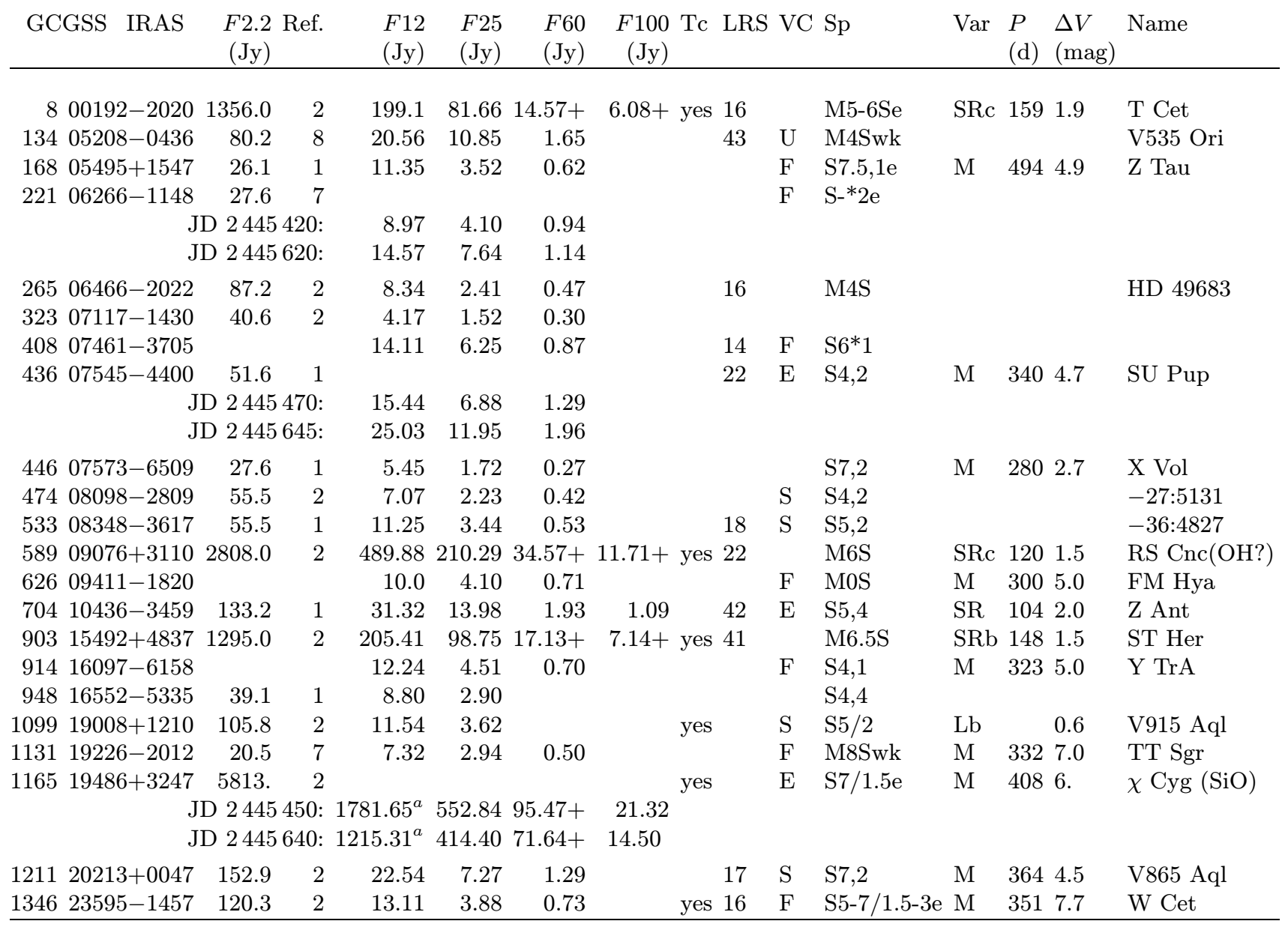

a: detector probably saturated

+: the "zero-crossing" flux density $F_{\mathrm{z}}$ has been adopted instead of the template flux density $F_{\mathrm{t}}$, indicating a possibly resolved shell (see Sect. 2.2). 
Table 1. continued

Region C: $[12]-[25]$ and [25] - [60] excesses, silicate emission common

\begin{tabular}{|c|c|c|c|c|c|c|c|c|c|c|c|c|c|c|c|c|}
\hline GC & GSS & IRAS & $\begin{array}{r}F 2.2 \\
(\mathrm{Jy}) \\
\end{array}$ & Ref. & $\begin{array}{l}F 12 \\
(\mathrm{Jy})\end{array}$ & $\begin{array}{l}F 25 \\
(\mathrm{Jy})\end{array}$ & $\begin{array}{l}F 60 \\
(\mathrm{Jy})\end{array}$ & $\begin{array}{r}F 100 \\
(\mathrm{Jy}) \\
\end{array}$ & $\mathrm{Tc}$ & LRS & $\mathrm{VC}$ & $\mathrm{Sp}$ & Var & $\begin{array}{l}P \\
(\mathrm{~d})\end{array}$ & $\begin{array}{l}\Delta V \\
(\mathrm{mag})\end{array}$ & Name \\
\hline 6 & 0013 & $35+4644$ & 37.4 & 2 & 15.96 & 6.24 & 1.57 & 0.85 & & 16 & $\mathrm{~F}$ & $\mathrm{~S} 4 / 7 \mathrm{e}$ & M & 346 & 6.9 & $\mathrm{X}$ And \\
\hline 9 & 0021 & $13+3817$ & 453.3 & 2 & 335.74 & 175.66 & $26.41+$ & 9.45 & yes & & $\mathrm{E}$ & S5-7/4-5e & $\mathrm{M}$ & 409 & 9.1 & R And $(\mathrm{SiO})$ \\
\hline 14 & 0044 & $45+3224$ & 96.5 & 2 & 37.75 & 18.58 & 3.97 & 2.10 & & 22 & $\mathrm{~F}$ & $\mathrm{~S} 6 / 2 \mathrm{e}$ & $\mathrm{M}$ & 430 & 7.8 & RW And \\
\hline 28 & 0115 & $59+7220$ & 147.4 & 2 & 343.76 & 192.52 & $30.14+$ & 10.88 & & 22 & $\mathrm{E}$ & $\mathrm{S} 4,6 \mathrm{e}$ & $\mathrm{M}$ & 612 & 8.2 & S Cas ( $\mathrm{SiO})$ \\
\hline 36 & 0126 & $36+5035$ & 34.1 & 6 & 6.59 & 1.93 & 0.45 & & & & & $\mathrm{~S} 6 / 8 \mathrm{e}$ & $\mathrm{M}$ & 355 & 5.3 & RZ Per \\
\hline 49 & 0214 & $43+4404$ & 275.7 & 2 & 166.42 & 70.94 & 14.01 & 5.43 & yes & 22 & $\mathrm{E}$ & $\mathrm{S} 7 / 1 \mathrm{e}$ & $\mathrm{M}$ & 396 & 7.9 & W And $(\mathrm{SiO})$ \\
\hline 149 & 0537 & $74+3153$ & 280.8 & 2 & 45.33 & 23.32 & 5.00 & & yes & 43 & & $\mathrm{M} 2 \mathrm{~S}$ & $\mathrm{Lc}$ & & 0.2 & NO Aur \\
\hline 231 & 0633 & $31+1415$ & 136.9 & 2 & 21.95 & 10.35 & $2.61^{a}$ & 3.90 & no & 42 & $\mathrm{~F}$ & S8 & SRa & 1145 & 1.4 & DY Gem \\
\hline \multirow[t]{3}{*}{283} & 0657 & $71+5524$ & 118.1 & 2 & & & & & & 16 & $\mathrm{~F}$ & $\mathrm{~S} 5 / 5 \mathrm{e}$ & M & 378 & 7.1 & R Lyn (SiO) \\
\hline & & & 2445 & 420 & 17.55 & 5.61 & 1.25 & & & & & & & & & \\
\hline & & JD & 2445 & $620:$ & 28.46 & 9.46 & 2.01 & & & & & & & & & \\
\hline \multirow[t]{3}{*}{307} & 0704 & $43+2246$ & 88.0 & 2 & & & & & yes & 16 & $\mathrm{~F}$ & $\mathrm{~S} 3,9 \mathrm{e}$ & $\mathrm{M}$ & 370 & 8.0 & R Gem \\
\hline & & $\mathrm{JD}$ & 2445 & 435 & 22.23 & 7.53 & 2.21 & 1.40 & & & & & & & & \\
\hline & & JD & 2445 & $620:$ & 42.33 & 16.81 & 3.73 & 1.48 & & & & & & & & \\
\hline \multirow{4}{*}{$\begin{array}{l}316 \\
326\end{array}$} & 0709 & $2+0735$ & & & 12.23 & 5.63 & 1.06 & 0.36 & & & $\mathrm{E}$ & $\mathrm{S}$ & M & 420 & 4.8 & WX CMi \\
\hline & 0714 & $49+0111$ & 70.5 & 2 & & & & & & 16 & & $\mathrm{M}$ & M & 395 & 7.4 & \\
\hline & & JD & D 2445 & $440:$ & 27.76 & 11.75 & 2.31 & 0.85 & & & & & & & & \\
\hline & & & 2445 & $625:$ & 15.77 & 7.21 & 1.69 & 1.03 & & & & & & & & \\
\hline 341 & 0719 & $97-1451$ & & & 20.24 & 10.47 & 1.86 & & & 27 & $\mathrm{E}$ & & $\mathrm{M}$ & 314 & 4.0 & TT CMa \\
\hline 347 & 0724 & $45+4605$ & 964.7 & 2 & 133.50 & 65.92 & $13.20+^{d}$ & $5.58+$ & yes & 23 & & M6S & $\mathrm{SRc}$ & 110 & 2.5 & $n(\mathrm{SiO})$ \\
\hline \multirow[t]{3}{*}{614} & 0933 & $38-5349$ & 68.0 & 1 & & & & & & 01 & $\mathrm{E}$ & $\mathrm{S} 7,8 \mathrm{e}$ & $\mathrm{M}$ & 408 & 3.0 & UU Vel \\
\hline & & JD & 2445 & 510 & 11.53 & 5.98 & 1.77 & & & & & & & & & \\
\hline & & & 2445 & $580:$ & 17.79 & 9.37 & & & & & & & & & & \\
\hline 649 & 0956 & $34-5837$ & & & 156.15 & 71.37 & 14.31 & & & 15 & $\mathrm{E}$ & S6.5/1- & $\mathrm{SRb}$ & 109 & 1.3 & Car \\
\hline \multirow{2}{*}{$\begin{array}{l}656 \\
816\end{array}$} & 1001 & $17-7012$ & 43.7 & 1 & 6.02 & 1.72 & 0.37 & & & & & & $\mathrm{M}$ & & 3 & \\
\hline & 1313 & $36-4426$ & 356.8 & 1 & 56.55 & 20.77 & 4.04 & 1.78 & & 43 & $\mathrm{C}$ & $\mathrm{S} 6 / 8=\mathrm{CS}$ & SR & 114 & 2.0 & Cen \\
\hline \multirow{2}{*}{$\begin{array}{l}821 \\
861\end{array}$} & 1324 & $40-5742$ & & & 13.40 & 6.27 & $b$ & & & 14 & $\mathrm{~F}$ & $\mathrm{~S} 6 * 3$ & $\mathrm{SR}$ & 198 & 1.9 & EE Cen \\
\hline & 1437 & $72-6106$ & 43.3 & 9 & 21.38 & 9.13 & & & & 15 & $\mathrm{E}$ & & & & & $\overline{C S}$ \\
\hline \multirow{2}{*}{$\begin{array}{l}872 \\
931\end{array}$} & 1503 & $30-4116$ & 111.8 & 1 & 21.81 & 9.23 & 2.18 & & & & $\mathrm{~F}$ & S7 & $\pi$ & 326 & 5.6 & $c$ \\
\hline & 1633 & $34-3107$ & 377.0 & 2 & 52.49 & 21.88 & $4.22^{c}$ & $b$ & & 16 & $\mathrm{E}$ & S8 & SRa & 194 & 3. & \\
\hline \multirow[t]{3}{*}{954} & 1700 & $1-3651$ & 453.3 & 1 & & & & & & 22 & $\mathrm{E}$ & $\mathrm{S} 7,2$ & $\mathrm{M}$ & 449 & 8.2 & RT Sco \\
\hline & & & D 2445 & 410 & 170.65 & 69.64 & 15.37 & & & & & & & & & \\
\hline & & & 2445 & 600: & 269.51 & 118.53 & 25.01 & & & & & & & & & \\
\hline- & 1708 & $31+6422$ & 377.0 & 2 & 61.96 & 25.52 & 4.87 & 2.27 & & & & & $\mathrm{Lb}$ & & 0.4 & $\mathrm{~T}$ \\
\hline 093 & 1857 & $75-0139$ & 37.7 & 1 & 17.18 & 5.92 & 1.83 & & & & & $\mathrm{SC}$ & M: & & & \\
\hline 1096 & 1858 & 36-1249 & 242.3 & 2 & 51.78 & 19.19 & 4.03 & & & 21 & $\mathrm{E}$ & & $\mathrm{M}$ & 395 & & \\
\hline \multirow[t]{3}{*}{1112} & 1911 & $11+2555$ & 18.7 & 6 & & & & & & 41 & & SC & M & 438 & 5.8 & $\mathrm{~S} \mathrm{~L}$ \\
\hline & & & D 2445 & 440 & 43.81 & 21.43 & 4.39 & & & & & & & & & \\
\hline & & & 2445 & $630:$ & 84.17 & 38.23 & 7.53 & 2.73 & & & & & & & & \\
\hline \multirow[t]{3}{*}{1115} & 1912 & $26-0708$ & 286.0 & 2 & & & & & & 22 & $\mathrm{E}$ & $\mathrm{S} 6 / 6 \mathrm{e}$ & $\mathrm{M}$ & 490 & 7.0 & W Aql (SiO) \\
\hline & & & & & $7.52^{e} 7$ & 719.87 & $32.67+$ & 30.01 & & & & & & & & \\
\hline & & JD & 244 & 30: & $57.16^{e}$ & 481.93 & $100.58+$ & 24.90 & & & & & & & & \\
\hline 1117 & 1913 & $33-1703$ & 166.1 & 2 & 42.27 & 14.62 & $4.76+4$ & $4.76+$ & $\mathrm{y}$ & & $\mathrm{F}$ & & M & 394 & 5.8 & \\
\hline \multirow{3}{*}{$\begin{array}{l}1150 \\
1159 \\
1175\end{array}$} & & & 107.7 & 2 & 107.44 & 52.86 & $12.22+$ & 5.52 & yes & 22 & $\mathrm{E}$ & & M & 426 & 8. & \\
\hline & 1945 & $51+0827$ & 15.3 & 7 & 7.69 & 3.53 & 0.84 & & & & I & $\mathrm{Se}$ & M & 607 & 2.0 & QU Aql \\
\hline & 1954 & & & & 7.59 & 3.80 & & & & 29 & $\mathrm{E}$ & & & & & V1407 Aql \\
\hline \multicolumn{5}{|c|}{$120020114+7702$} & & & & & & & I & $\mathrm{S} 5 / 6 \mathrm{e}$ & $\mathrm{M}$ & 326 & 6.9 & SZ Cep \\
\hline \multirow{2}{*}{\multicolumn{5}{|c|}{$\begin{array}{l}\text { JD } 2445394: \\
\text { JD } 2445580 \text { : }\end{array}$}} & 7.27 & 2.71 & 0.68 & & & & & & & & & \\
\hline & & & & & 9.67 & 3.45 & 0.72 & & & & & & & & & \\
\hline 1268 & 2117 & $72-4819$ & 14.9 & 1 & 7.15 & 2.34 & 0.63 & & & & I & $\mathrm{S} 2,5$ & & & & $-48: 13866$ \\
\hline
\end{tabular}

a: close weak source

b: profile badly distorted by close source

c: close weak source apparent on some scans

d: moves to Region B after removing the extended shell contribution

e: detector possibly saturated

+ : the "zero-crossing" flux density $F_{\mathrm{z}}$ has been adopted instead of the template flux density $F_{\mathrm{t}}$, indicating a possibly resolved shell (see Sect. 2.2). 
Table 1. continued

Region C (continued)

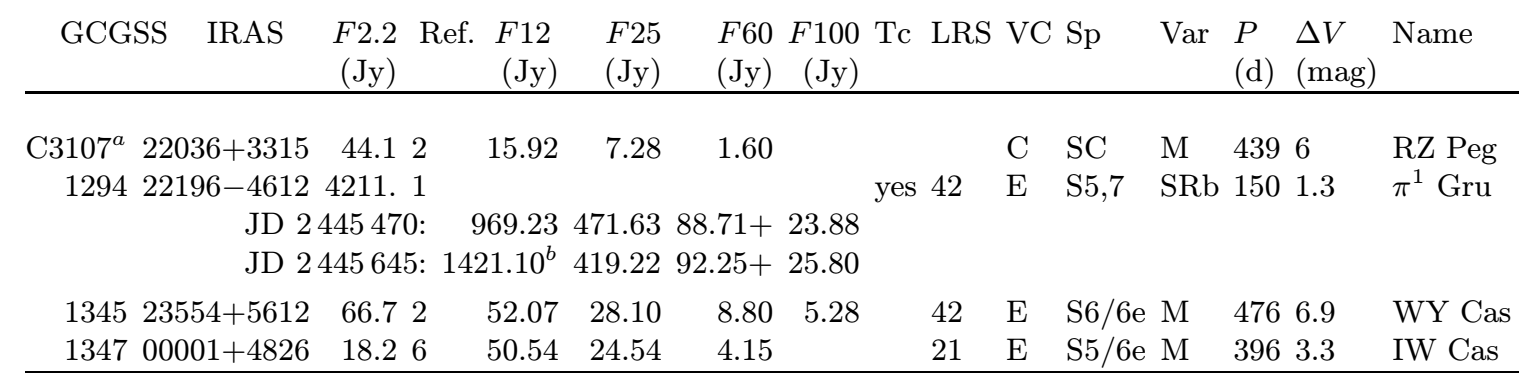

a: star number from the General Catalogue of Carbon Stars (Stephenson 1989)

b: detector possibly saturated

+: the "zero-crossing" flux density $F_{\mathrm{z}}$ has been adopted instead of the template flux density $F_{\mathrm{t}}$, indicating a possibly resolved shell (see Sect. 2.2).

Region D: No silicate emission, weak $K-[12]$ excess, some [25] - [60] excess

\begin{tabular}{|c|c|c|c|c|c|c|c|c|c|c|c|c|}
\hline GCGSS IRAS & $\begin{array}{l}F 2.2 \mathrm{H} \\
(\mathrm{Jy})\end{array}$ & $\begin{array}{r}\text { Ref. } F 12 \\
(\mathrm{Jy})\end{array}$ & $\begin{array}{l}F 25 \\
(\mathrm{Jy}) \\
\end{array}$ & $\begin{array}{l}F 60 \\
(\mathrm{Jy}) \\
\end{array}$ & $\begin{array}{l}F 100 \mathrm{Tc} \\
(\mathrm{Jy})\end{array}$ & LRS & $\mathrm{V}$ & $\mathrm{Sp}$ & Var & $\begin{array}{l}P \\
(\mathrm{~d}) \\
\end{array}$ & $\begin{array}{l}\Delta V \\
(\mathrm{mag})\end{array}$ & Name \\
\hline $1200435+4758$ & 64.92 & 8.47 & 2.49 & $a$ & ${ }^{a}$ yes & 01 & $\mathrm{~S}$ & $\mathrm{~S} 5 / 3 \mathrm{e}$ & $\mathrm{M}$ & 277 & 7.7 & U Cas \\
\hline $1700486+3406$ & 36.35 & 4.56 & 1.44 & 0.35 & & & & $\mathrm{~S} 6 / 3 \mathrm{e}$ & M & 328 & 7.2 & RR And \\
\hline $2000578+5620$ & 169.22 & $2 \quad 21.87$ & 7.81 & 2.43 & & 16 & $\mathrm{~F}$ & $\mathrm{~S} 6 / 3$ & $\mathrm{SRb}$ & 136 & 1.8 & V365 Cas \\
\hline $2901186+6634$ & 46.62 & 5.10 & 1.90 & 0.42 & & & & & & & & \\
\hline $5702228+3753$ & 95.62 & 9.89 & 2.80 & $0.66+$ & 0.70 & 01 & $\mathrm{~S}$ & $\mathrm{~S} 8,8$ & SR & 159 & 1.1 & BI And \\
\hline $10304352+6602$ & 398.52 & 242.56 & 11.64 & 3.70 & 2.21 yes & 17 & $\mathrm{~S}$ & $\mathrm{~S} 4,7 \mathrm{e}$ & $\mathrm{M}$ & 373 & 7.1 & T Cam \\
\hline $11604543+4829$ & 175.52 & $2 \quad 12.81$ & 4.27 & 1.38 & yes & & $\mathrm{F}$ & $\mathrm{S} 5,8$ & $\mathrm{SRb}$ & 182 & 0.7 & TV Aur \\
\hline $16005440+1753$ & & 4.72 & 1.55 & 0.50 & & & & & $\mathrm{SRa}$ & 364 & 2.5 & EI Tau \\
\hline $23706347+0057$ & 51.12 & 6.18 & 2.21 & 0.85 & & & & & $\mathrm{Lb}$ & & 0.9 & CX Mon \\
\hline $31207095+6853$ & 138.22 & $2 \quad 14.57$ & 5.98 & $1.94+$ & 1.87 yes & & & M5S & $\mathrm{Lb}$ & & 0.6 & AA Cam \\
\hline $38707399-1045$ & 177.22 & $\begin{array}{ll}2 & 19.48\end{array}$ & 7.25 & 2.08 & & 18 & $\mathrm{~S}$ & $\mathrm{~S} 3,6$ & $\mathrm{SRb}$ & & 2.8 & SU Mon \\
\hline $52408308-1748$ & 65.52 & 5.85 & 2.00 & 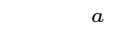 & & & & & M & 250 & 1.5 & SZ Pyx \\
\hline $55608461-7051$ & 53.01 & 5.70 & 1.49 & 0.46 & 0.33 & & & $\mathrm{SC}$ & Lb: & & 0.4 & UX Vol \\
\hline $61709358-6536$ & 39.51 & 4.00 & 1.22 & 0.27 & & & & $\mathrm{~S} 7,8$ & & & & \\
\hline $69610389-5149$ & 109.71 & $1 \quad 10.61$ & 3.21 & 1.11 & & 18 & $\mathrm{~S}$ & $\mathrm{~S} 5,2$ & $\mathrm{Lb}$ & & 0.4 & HP Vel \\
\hline $78712135-5600$ & & 19.78 & 6.55 & 1.65 & & 18 & & $\mathrm{SC6} / 8 \mathrm{e}$ & $\mathrm{M}$ & & 4.3 & BH Cru \\
\hline $80312417+6121$ & 39.53 & 4.39 & 1.38 & 0.35 & 0.39 yes & & & $\mathrm{S} 3 / 6 \mathrm{e}$ & $\mathrm{M}$ & 226 & 5.6 & S UMa \\
\hline $83013440-5306$ & 129.51 & $1 \quad 15.60$ & 5.66 & 2.08 & & 18 & & $\mathrm{SC}$ & $\mathrm{Lb}$ & & 1. & $\mathrm{Al}$ \\
\hline $83413477-6009$ & 387.61 & 149.16 & 14.57 & 3.42 & & 17 & $\mathrm{~S}$ & $\mathrm{~S} 8,5$ & $\mathrm{SR}$ & 307 & 3. & Cen \\
\hline $904 \quad 15548-7420$ & 58.11 & 6.12 & 1.93 & 0.65 & 0.59 & & & $\mathrm{SC}$ & SRa & 152 & 3.5 & VY Aps \\
\hline $923 \quad 16209-2808$ & 71.22 & 7.71 & 4.10 & 1.27 & & & & & & & & \\
\hline $94016472-6056$ & 49.21 & 5.71 & 2.06 & 0.72 & & & & $\mathrm{~N}$ & IVI: & & 2 & \\
\hline $978 \quad 17206-2826$ & 319.42 & $2 \quad 39.93$ & 11.48 & 2.72 & & 17 & $\mathrm{~S}$ & $\mathrm{~S} 5,4$ & $\mathrm{SRb}$ & 320 & 1. & V521 Oph \\
\hline $17553+4521$ & 545.02 & $2 \quad 55.41$ & 17.50 & $4.14+^{d}$ & $3.20+$ yes & 17 & & MII-III & $\mathrm{SRb}$ & 120 & 0.9 & OP Her \\
\hline $101117562-1133$ & & 8.45 & 3.30 & & & 01 & & & & & & \\
\hline $105618310-3541$ & 53.07 & 5.99 & 2.05 & 0.62 & & & & & L & & 0 . & $V$ \\
\hline $114119311+2332$ & 258.22 & $2 \quad 32.48$ & 13.87 & 3.06 & & 16 & $\mathrm{~F}$ & $\mathrm{~S} 6 / 5$ & $\mathrm{Lb}$ & & 3. & $\mathrm{EP}$ Vul $(\mathrm{SiO})$ \\
\hline $118820026+3640$ & 356.82 & $2 \quad 40.22$ & 15.45 & 5.37 & yes & 31 & $\mathrm{~S}$ & $\mathrm{~S} 7,5$ & $\mathrm{SRb}$ & 212 & 3.0 & AA Cyg \\
\hline $131022479+5923$ & 91.32 & $2 \quad 10.03$ & 3.25 & $a$ & & & $\mathrm{~F}$ & $\mathrm{~S} 6 / 2$ & $\mathrm{SRb}$ & 60 & 1.4 & CV Cep \\
\hline $130822476+4047$ & 766.32 & 2100.51 & 32.66 & $10.25+$ & $10.07+$ & 16 & $\mathrm{~S}$ & $\mathrm{~S} 7.5 / 1$ & $\mathrm{SRb}$ & 174 & 1.7 & RX Lac \\
\hline $133923380+7009$ & 60.92 & $2 \quad 7.95$ & 2.89 & 0.86 & & & $\mathrm{~F}$ & M6S & & & & \\
\hline
\end{tabular}

a: profile badly distorted by close source

c: strong cirrus contamination

d: moves to Region B after removing the extended shell contribution

+ : the "zero-crossing" flux density $F_{\mathrm{z}}$ has been adopted instead of the template flux density $F_{\mathrm{t}}$, indicating a possibly resolved shell (see Sect. 2.2). 
Table 1. continued

Region E: Strong [25] - [60] excess, many resolved shells

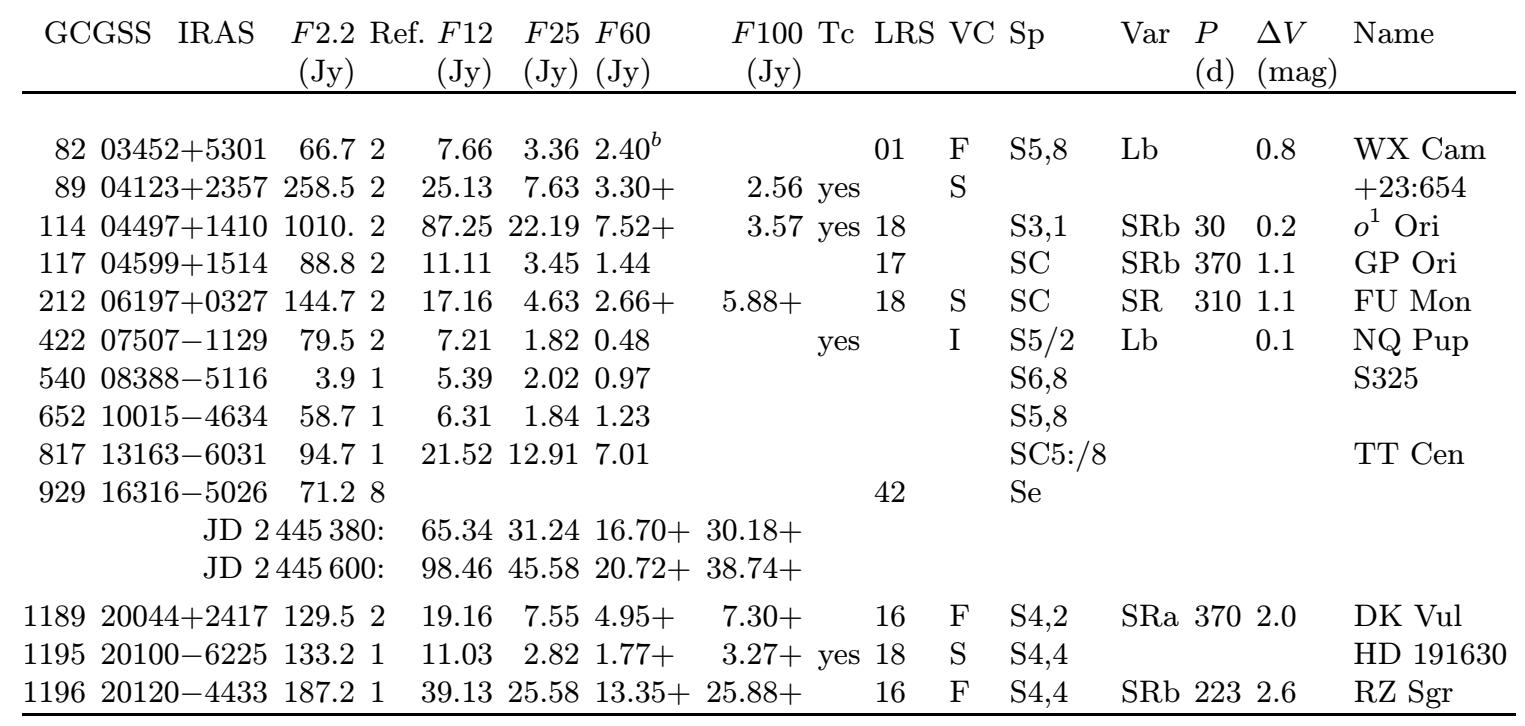

b: close weak source apparent on some scans

+: the "zero-crossing" flux density $F_{\mathrm{z}}$ has been adopted instead of the template flux density $F_{\mathrm{t}}$, indicating a possibly resolved shell (see Sect. 2.2). 
Table 2. S stars whose circumstellar envelopes are resolved at $60 \mu \mathrm{m}$, listed according to the region to which they belong in the $(K-[12],[25]-[60])$ diagram (see Fig. 3). In column Rem, YPK+ and YPK - denote stars found to be extended or not, respectively, by Young et al. (1993a). Stars with no YPK label were not examined by Young et al. (1993a). The mass loss rates and wind velocities listed in columns $\dot{M}$ and $V_{\mathrm{e}}$, respectively, are taken from Table 6

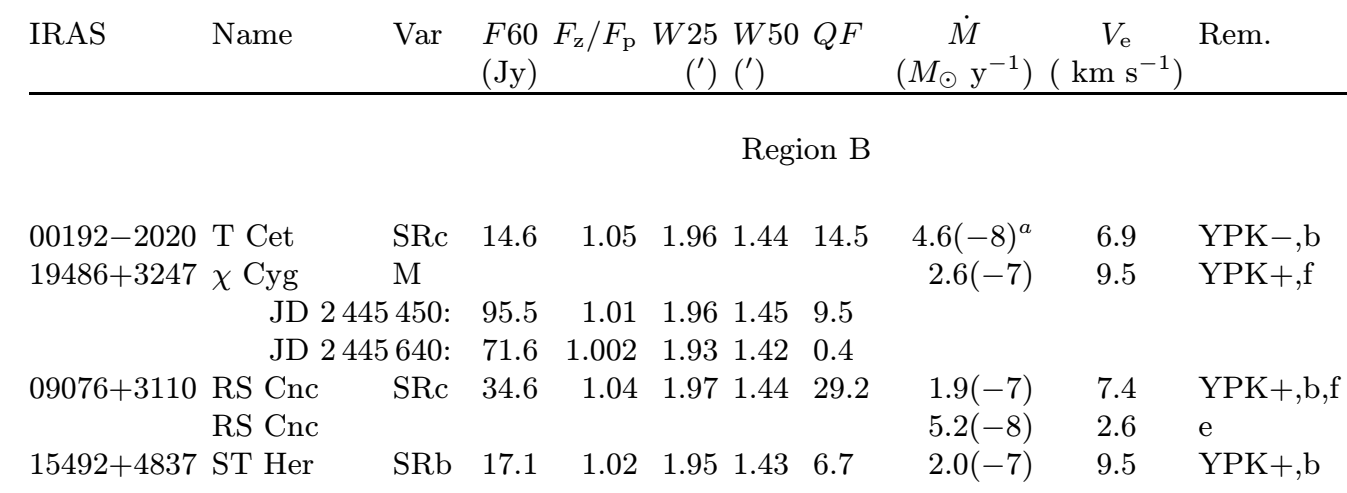

Region C

$\begin{array}{lllrrrrrrrl}00213+3817 & \text { R And } & \text { M } & 26.4 & 1.05 & 2.01 & 1.47 & 30.6 & 8.6(-7) & 9.3 & \text { YPK- } \\ 01159+7220 & \text { S Cas } & \text { M } & 30.1 & 1.01 & 1.94 & 1.42 & 10.4 & 4.0(-6) & 20.0 & \text { YPK- } \\ 07245+4605 & \text { Y Lyn } & \text { SRc } & 13.2 & 1.15 & 2.00 & 1.45 & 30.9 & 2.3(-7) & 8.6 & \text { YPK+,d } \\ 19126-0708 & \text { W Aql } & \text { M } & & & & & & 1.3(-5) & 18.7 & \text { YPK- } \\ & \text { JD 2 } 445 & 440: & 132.7 & 1.006 & 1.93 & 1.44 & 5.8 & & & \\ \text { JD 2 } 445630: & 100.6 & 1.02 & 1.95 & 1.44 & 7.5 & & & \\ 19133-1703 \text { T Sgr } & \text { M } & 4.8 & 1.11 & 2.05 & 1.48 & 8.0 & 4.0(-7) & 14.1 & \text { b } \\ 19354+5005 \text { R Cyg } & \text { M } & 12.2 & 1.03 & 1.96 & 1.44 & 10.0 & 1.7(-6) & 10.5 & \text { YPK+,f } \\ 22196-4612 & \pi^{1} \text { Gru } & \text { SRb } & & & & & & 4.6(-7) & 11.0 & \text { YPK+,f }\end{array}$

JD $2445470: \quad 88.7 \quad 1.04 \quad 1.98 \quad 1.44 \quad 37.9$

JD $2445645: \quad 92.2 \quad 1.07 \quad 2.02 \quad 1.49 \quad 38.3$

Region D

\begin{tabular}{|c|c|c|c|c|c|c|c|c|c|c|}
\hline $02228+3753$ & BI And & SR & 0.7 & - & 2.17 & 1.58 & 2.6 & & & \\
\hline $07095+6853$ & AA Cam & $\mathrm{Lb}$ & 1.9 & - & 2.08 & 1.57 & 4.1 & $2.9(-7)$ & 17.9 & \\
\hline $17553+4521$ & OP Her & $\mathrm{SRb}$ & 4.1 & 1.15 & 2.16 & 1.53 & 42.4 & & & $\mathrm{~b}$ \\
\hline $22476+4047$ & RX Lac & $\mathrm{SRb}$ & 10.2 & 1.16 & 2.11 & 1.52 & 23.6 & $4.0(-8)$ & 3.4 & $\mathrm{~b}$ \\
\hline \multicolumn{11}{|c|}{ Region E } \\
\hline $04123+2357$ & $\mathrm{BD}+23^{\circ} 654$ & - & 3.3 & 1.20 & 2.18 & 1.53 & 7.2 & & & \\
\hline $04497+1410$ & $o^{1}$ Ori & $\mathrm{SRb}$ & 7.5 & 1.48 & 2.89 & 1.71 & 34.8 & $<2.0(-7)$ & & $\mathrm{c}$ \\
\hline $06197+0327$ & FU Mon & SR & 2.7 & 1.40 & 2.42 & 1.62 & 12.6 & $1.0(-7)$ & 2.8 & $\mathrm{~b}$ \\
\hline \multirow[t]{3}{*}{$16316-5026$} & S929 & - & & & & & & & & \\
\hline & JD 2445 & $5380:$ & 16.7 & 1.50 & 3.08 & 1.81 & 24.8 & & & $\mathrm{~b}$ \\
\hline & JD 244 & 5600: & 20.7 & 1.89 & - & 1.87 & 24.1 & & & $\mathrm{~b}$ \\
\hline $20044+2417$ & DK Vul & $\mathrm{SRa}$ & 4.9 & 1.22 & 2.32 & 1.60 & 25.3 & $2.9(-7)$ & 5.0 & $\mathrm{~b}$ \\
\hline $20100-6225$ & HD 191630 & - & 1.8 & 1.35 & 2.57 & 1.67 & 24.0 & $<1.0(-6)$ & & $\mathrm{b}, \mathrm{c}$ \\
\hline $20120-4433$ & RZ Sgr & $\mathrm{SRb}$ & 13.3 & 1.26 & 2.28 & 1.58 & 62.1 & $2.1(-6)$ & 8.8 & $\mathrm{YPK}+, \mathrm{b}, \mathrm{d}$ \\
\hline
\end{tabular}

Notes:

a: numbers between parentheses refer to power of ten

b: $100 \mu \mathrm{m}$ profile wider than point-source template

c: listed as extended at $60 \mu \mathrm{m}$ in the IRAS Small Scale Structure Catalogue

d: listed as extended at $100 \mu \mathrm{m}$ in the IRAS Small Scale Structure Catalogue

e: two-components wind

f: circumstellar shell resolved in CO (Sahai 1992; Stanek et al. 1995). 
Table 3. Masers in S stars

\begin{tabular}{|c|c|c|c|c|c|}
\hline Star & IRAS & $\mathrm{SiO}$ & $\mathrm{H}_{2} \mathrm{C}$ & $\mathrm{OH}$ & Refs. \\
\hline & \multicolumn{5}{|c|}{ Region A } \\
\hline BD Cam & $03377+6303$ & - & $\mathrm{N}$ & - & B96 \\
\hline NZ Gem & $07392+1419$ & & $\mathrm{~N}$ & - & B96 \\
\hline HR Peg & $22521+1640$ & $\mathrm{~N}$ & $\mathrm{~N}$ & - & B96, Z89 \\
\hline \multirow[t]{2}{*}{ GZ Peg } & $23070+0824$ & $\mathrm{~N}$ & $\mathrm{~N}$ & - & D73, H89 \\
\hline & \multicolumn{5}{|c|}{ Region B } \\
\hline T Cet & 00192-2020 & $\mathrm{N}$ & $\mathrm{N}$ & $\mathrm{N}$ & $\begin{array}{l}\text { A } 89 a, \text { A90, D76, } \\
\text { H94, L78, P71 }\end{array}$ \\
\hline V535 Ori & 05208-0436 & - & $\mathrm{N}$ & $\mathrm{N}$ & D89, H91 \\
\hline Z Tau & $05495+1549$ & $\mathrm{~N}$ & - & $\mathrm{N}$ & B78, J91 \\
\hline RS Cnc & $09076+3110$ & $\mathrm{~N}$ & $\mathrm{~N}$ & $\mathrm{Y} ?$ & $\begin{array}{lll}\text { D73, } & \text { D76, } & \text { A92, } \\
\text { D82, } & \text { K77, L78, } \\
\text { L90, } & \text { P92, } & \text { R76 }\end{array}$ \\
\hline Z Ant & 10436-3459 & - & - & $\mathrm{N}$ & H91 \\
\hline ST Her & $15492+4837$ & - & $\mathrm{N}$ & $\mathrm{N}$ & $\begin{array}{l}\text { D73, D76, D82, } \\
\text { P79 }\end{array}$ \\
\hline S Her & $16496+1501$ & $\mathrm{~N}$ & $\mathrm{~N}$ & & $\begin{array}{l}\mathrm{B} 77 \mathrm{a}, \mathrm{B} 84, \quad \mathrm{~B} 87, \\
\mathrm{~B} 90, \mathrm{E} 88, \quad \mathrm{~S} 81\end{array}$ \\
\hline$\chi$ Cyg & $19486+3249$ & $\mathrm{Y}$ & $\mathrm{N}$ & $\mathrm{N}$ & $\begin{array}{l}\text { A89b, A92, } \\
\text { B87, B94, C83a, } \\
\text { C96, D76, J84, } \\
\text { J85, J87, J91, } \\
\text { N85, O85, P92 }\end{array}$ \\
\hline \multirow[t]{2}{*}{ W Cet } & $23595-1457$ & $\mathrm{~N}$ & $\mathrm{~N}$ & - & $\begin{array}{l}\text { B77a，B84，L76, } \\
\text { L78 }\end{array}$ \\
\hline & \multicolumn{5}{|c|}{ Region C } \\
\hline $\mathrm{R}$ And & $00213+3817$ & $\mathrm{Y}$ & $\mathrm{N}$ & $\mathrm{N}$ & $\begin{array}{l}\text { B } 77 a, \quad B 78, \text { B } 87, \\
\text { B94, B96, C96, } \\
\text { D78, F75, J91, } \\
\text { T94 }\end{array}$ \\
\hline RW And & $00445+3224$ & $\mathrm{~N}$ & $\mathrm{~N}$ & $\mathrm{~N}$ & $\begin{array}{l}\text { B77a, B78, C83b, } \\
\text { C96, F75, F78, } \\
\text { K77, W72 }\end{array}$ \\
\hline $\mathrm{S}$ Cas & $01159+7220$ & $\mathrm{Y}$ & $\mathrm{N}$ & $\mathrm{N}$ & $\begin{array}{l}\text { B77a, B94, F73, } \\
\text { T94 }\end{array}$ \\
\hline RZ Per & $01266+5035$ & $\mathrm{~N}$ & - & - & B77a \\
\hline W And & $02143+4404$ & $\mathrm{Y}$ & $\mathrm{N}$ & $\mathrm{N}$ & $\begin{array}{l}\text { A89b, B77a, B87, } \\
\text { B94, C96, D96, } \\
\text { D78, K77, O80, } \\
\text { T94, W72 }\end{array}$ \\
\hline NO Aur & $05374+3153$ & - & $\mathrm{N}$ & $\mathrm{N}$ & B75, B81, B96 \\
\hline R Lyn & $06571+5524$ & $\mathrm{Y}$ & $\mathrm{N}$ & - & $\begin{array}{l}\text { B77a， B94，K84, } \\
\text { S81 }\end{array}$ \\
\hline R Gem & $07043+2246$ & $\mathrm{~N}$ & $\mathrm{~N}$ & $\mathrm{~N}$ & $\begin{array}{l}\text { B77a, B78, B96, } \\
\text { J91, L78, W72 }\end{array}$ \\
\hline RR Mon & $07149+0111$ & $\mathrm{~N}$ & - & $\mathrm{N}$ & $\mathrm{K} 77, \mathrm{~L} 78$ \\
\hline TT CMa & 07197-1451 & $\mathrm{N}$ & $\mathrm{N}$ & $\mathrm{N}$ & B90, B96, H91 \\
\hline Y Lyn & $07245+4605$ & $\mathrm{Y}$ & $\mathrm{N}$ & $\mathrm{N}$ & $\begin{array}{l}\text { A90, B75, B81, } \\
\text { D73, }\end{array}$ \\
\hline RR Car & 09564-5837 & $\mathrm{N}$ & $\mathrm{N}$ & - & H94, L77 \\
\hline ST Sco & $16334-3107$ & $\mathrm{~N}$ & $\mathrm{~N}$ & $\mathrm{~N}$ & $\begin{array}{l}\text { B77a, B94, J88, } \\
\text { L77, W72 }\end{array}$ \\
\hline RT Sco & $17001-3651$ & $\mathrm{~N}$ & $\mathrm{~N}$ & $\mathrm{~N}$ & $\begin{array}{l}\text { B77b, C71, L76, } \\
\text { L77, L } 78, \quad S 88\end{array}$ \\
\hline TV Dra & $17081+6422$ & $\mathrm{~N}$ & - & $\mathrm{N}$ & B94, W72 \\
\hline ST Sgr & $18586-1249$ & $\mathrm{~N}$ & $\mathrm{~N}$ & - & B77a \\
\hline W Aql & 19126-0708 & $\mathrm{Y}$ & $\mathrm{N}$ & $\mathrm{N}$ & $\begin{array}{l}\text { B } 77 a, \quad B 84, \text { B87, } \\
\text { B94, D89, L78, } \\
\text { W72, Z79 }\end{array}$ \\
\hline
\end{tabular}

Table 3. continued

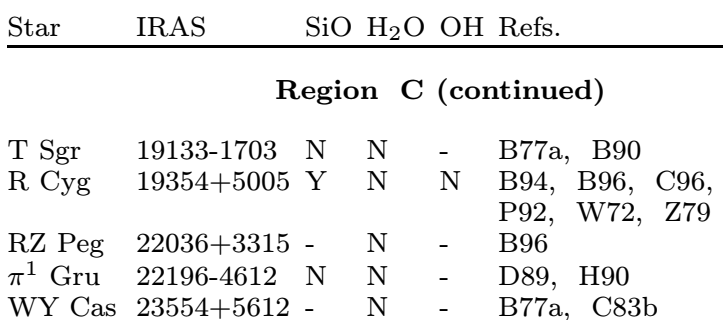

\section{Region D}

U Cas $00435+4758$ N N $\quad$ B $77 a$, C83b, J91 RR And $00486+3406 \mathrm{~N} \quad \mathrm{~N} \quad \mathrm{~N} \quad \begin{aligned} & \mathrm{S} 81 \\ & \mathrm{~B} 77 \mathrm{a},\end{aligned}$

S29 $01186+6634-\mathrm{N}-\mathrm{C} 83 \mathrm{~b}$

BI And $02228+3753$ - N - C83b

T Cam $04352+6602 \mathrm{~N} N-\mathrm{B} 77 \mathrm{a}, \mathrm{B} 94, \mathrm{D} 76$,

TV Aur $04543+4829 \mathrm{~N}-\quad-\quad$ B77a, B94

EI Tau $05440+1753 \mathrm{~N}-\quad-\mathrm{B} 77 \mathrm{a}$

AA Cam $07095+6853$ - N $\quad$ - B96

SU Mon 07399-1045 N - $\quad$ - B94

SZ Pyx $08308-1748-\mathrm{N} \quad-\mathrm{C} 83 \mathrm{~b}$

S UMa $12417+6121 \mathrm{~N} N \quad$ - B77a, B96, D78

VX Cen 13477-6009 N - $\quad$ - P92

OP Her $17553+4521$ - $\mathrm{N} \quad-\quad \mathrm{B} 96$

EP Vul $19311+2332 \mathrm{Y}-\mathrm{N} \quad \mathrm{B} 94, \mathrm{R} 76$

AA Cyg $20026+3640 \mathrm{~N}$ N $-\mathrm{B} 77 \mathrm{a}, \mathrm{B} 94, \mathrm{~B} 96$, D82

RX Lac $22476+4047-\quad \mathrm{N} \quad \mathrm{N} \quad \mathrm{D} 73, \mathrm{D} 82, \mathrm{~W} 72$

\section{Region E}

$\begin{array}{lllllll}o^{1} \text { Ori } 04497+1410- & \mathrm{N} & \mathrm{N} & \mathrm{B} 96, & \mathrm{D} 73, & \mathrm{P} 71\end{array}$

GP Ori $04599+1514 \mathrm{~N} \quad-\quad-\quad$ L78

FU Mon $06197+0327$ N $\quad-\quad$ B 77 a

RZ Sgr 20120-4433 - $\quad-\quad$ N C71 
References to Table 3. The parentheses at the end of each reference indicate which maser is probed by the paper

A89a: Allen D.A., Hall P.J., Norris R.P., Troup E.R., Wark R.M., Wright A.E., 1989, MNRAS 236, 363 (SiO)

A89b: Alcolea J., Bujarrabal V., Gallego J., 1989, A\&A 211, $187(\mathrm{SiO})$

A90: Alcolea J., Bujarrabal V., Gomez-Gonzalez J., 1990, A\&A $231,431(\mathrm{SiO})$

A92: Alcolea J., Bujarrabal V., 1992, A\&A 253, 475 (SiO)

B75: Bowers P.F., 1975, AJ 80, $512(\mathrm{OH})$

B77a: Blair G.N., Dickinson D.F., 1977, ApJ 215, 552 (SiO, $\mathrm{H}_{2} \mathrm{O}$ )

B77b: Bowers P.F., Kerr F.J., 1977, A\&A 57, 115 (OH)

B78: Bowers P.F., Sinha R.P., 1978, AJ 83, $955(\mathrm{OH})$

B81: Bowers P.F., 1981, AJ 86, 1930 (OH)

B84: Bowers P.F., Hagen W., 1984, ApJ 285, $637\left(\mathrm{H}_{2} \mathrm{O}\right)$

B87: Bujarrabal V., Planeses P., del Romero A., 1987, A\&A $175,164(\mathrm{SiO})$

B90: Benson P.J., Little-Marenin I.R., Woods T.C., et al., 1990, ApJS 74, $911\left(\mathrm{SiO}, \mathrm{H}_{2} \mathrm{O}, \mathrm{OH}\right)$

B94: Bieging J.H., Latter W.B., 1994, A\&A 422, 765 (SiO)

B96: Benson P.J., Little-Marenin I.R., 1996, ApJS 106, 579 $\left(\mathrm{H}_{2} \mathrm{O}\right)$

C71: Caswell J.L., Robinson B.J., Dickel H.R., 1971, Ap. Lett. 9, $61(\mathrm{OH})$

C83a: Clemens D.P., Lane A.P., 1983, ApJ 266, L117 (SiO)

C83b: Crocker D.A., Hagen W., 1983, A\&AS 54, $405\left(\mathrm{H}_{2} \mathrm{O}\right)$

C96: Cho S.H., Kaifu N., Ukita N., 1996, A\&AS 115, 117 (SiO)

D73: Dickinson D.F., Bechis K.P., Barrett, A.H., 1973, ApJ 180, $831\left(\mathrm{H}_{2} \mathrm{O}\right)$

D76: Dickinson D.F., 1976, ApJS 30, $259\left(\mathrm{H}_{2} \mathrm{O}\right)$

D78: Dickinson D.F., Snyder L.E., Brown L.W., Buhl D., 1978, AJ 83, 36 ( $\mathrm{SiO})$

D82: Dickinson D.F., Dinger A.S., 1982, ApJ 254, $136\left(\mathrm{H}_{2} \mathrm{O}\right)$

D89: Deguchi S., Nakada Y., Forster J.R., 1989, MNRAS 239, $825\left(\mathrm{H}_{2} \mathrm{O}\right)$

E88: Engels D., Schmid-Burgk J., Walmsley C.M., 1988, A\&A 191, 283

F73: Fillit R., Foy R., Gheudin M., 1973, Ap. Letters 14, 135 $(\mathrm{OH})$

F75: Foy R., Heck A., Mennessier M.O., 1975, A\&A 43, 175 $(\mathrm{OH})$

F78: Fix J.D., Weisberg J.M., 1978, ApJ 220, 836 (OH)

H89: Heske A., 1989, A\&A 208, 77 (SiO)

H90: Haikala L.K., 1990, A\&AS 85, 875 (SiO)

H91: te Lintel Hekkert P., Caswell J.S., Habing H.J., Haynes R.F., Norris R.P., 1991, A\&AS 90, $327(\mathrm{OH})$

H94: Haikala L.K., Nyman L.A., Forsstrom V., 1994, A\&AS 103, $107(\mathrm{SiO})$

J84: Jewell P.R., Batrla W., Walmsley C.M., Wilson T.L., 1984, A\&A 130, L1 (SiO)

J85: Jewell P.R., Walmsley C.M., Wilson T.L., Snyder L.E., 1985, ApJ 298, L55 (SiO)

J87: Jewell P.R., Dickinson D.F., Snyder L.E., Clemens D.P., 1987, ApJ 323, $749(\mathrm{SiO})$

J91: Jewell P.R., Snyder L.E., Walmsley C.M., Wilson T.L., Gensheimer P.D., 1991, A\&A 242, 211 (SiO)

K77: Kolena J., Pataki L., 1977, AJ 82, 150 (OH)
References to Table 3 (continued).

K84: Kuiper T.B.H., Swanson P.N., Dickinson D.F., et al., 1984, ApJ 286, $310\left(\mathrm{H}_{2} \mathrm{O}\right)$

L76: Lépine J.R.D., Paes de Barros M.H., Gammon R.H., 1976, A\&A 48, $269\left(\mathrm{H}_{2} \mathrm{O}\right)$

L77: Lépine J.R.D., Paes de Barros M.H., 1977, A\&A 56, 219 $\left(\mathrm{H}_{2} \mathrm{O}\right)$

L78: Lépine J.R.D., Le Squeren A.M., Scalise E., 1978, ApJ $225,869(\mathrm{SiO})$

L90: Lewis B.M., Eder J., Terzian Y., 1990, ApJ 362, 634 (OH)

N85: Nyman L.A., Olofsson H., 1985, A\&A 147, 309 (SiO)

O80: Olnon F.M., Winnberg A., Matthews H. E., Schultz G.V., 1980, A\&AS 42, $119\left(\mathrm{OH}, \mathrm{H}_{2} \mathrm{O}\right)$

O95: Olofsson H., Rydbeck O.E.H., Nyman L.A., 1985, A\&A 150, $169(\mathrm{SiO})$

P71: Pashchenko M.I., Slysh V., Strukov I., et al., 1971, A\&A $11,482(\mathrm{OH})$

P79: Pashchenko M.I., Rudnitskij G.M., 1979, Astron. Tsirk. 1040, 4

P92: Patel N.A., Joseph A., Ganesan R., 1992, J. Astron. Astrophys. 13, 241 ( $\mathrm{SiO})$

R76: Rudnitskij G.M., 1976, Soviet AJ 20, 693 (OH)

S81: Spencer J.H., Winnberg A., Olnon F.M., et al., 1981, AJ 86, $392(\mathrm{SiO})$

S88: Sivagnanam P., Le Squeren A.M., Foy R., 1988, A\&A 206, $285(\mathrm{OH})$

T94: Takaba H., Ukita N., Miyaji T., Miyoshi M., 1994, PASJ $46,629\left(\mathrm{H}_{2} \mathrm{O}\right)$

W72: Wilson W.J., Barrett A.H., 1972, A\&A 17, 385 (OH)

Z79: Zuckerman B., 1979, ApJ 230, 442 (SiO) 
Table 4. S stars observed in $\mathrm{CO}$ at the Caltech Submillimeter Observatory

\begin{tabular}{|c|c|c|c|c|c|c|c|c|c|c|}
\hline GCGSS & Other & $\alpha(1950)$ & $\delta(1950)$ & $l$ & $b$ & $\mathrm{SpT}$ & Var & $\begin{array}{c}P \\
(\mathrm{~d}) \\
\end{array}$ & $\begin{array}{c}V_{\mathrm{LSR}} \\
\left(\mathrm{km} \mathrm{s}^{-1}\right) \\
\end{array}$ & $\begin{array}{c}\mathrm{rms} \\
(\mathrm{mK}) \\
\end{array}$ \\
\hline 89 & HD 26816 & 041223.7 & +235715 & 171.6 & -19.0 & S & $\mathrm{Lb}:$ & - & - & 17 \\
\hline 117 & GP Ori & 045956.7 & +151458 & 186.0 & -15.7 & $\mathrm{SC} 7 / 8$ & $\mathrm{SRb}$ & $370:$ & $+86,+92$ & 37 \\
\hline 149 & NO Aur & 053726.8 & +315343 & 176.9 & +0.7 & M2IIIS & $\mathrm{Lc}$ & - & $-7,-3$ & 13 \\
\hline 212 & FU Mon & 061946.1 & +032700 & 206.6 & -4.9 & $\mathrm{SC}$ & SR & 310 & $-46,-39$ & 10 \\
\hline 283 & R Lyn & 065710.8 & +552406 & 161.2 & +23.4 & $\mathrm{~S} 5 / 5 \mathrm{e}$ & Mira & 378 & $+9,+28$ & 18 \\
\hline 422 & NQ Pup & 075043.6 & -112941 & 230.5 & +8.04 & $\mathrm{~S} 5 / 2$ & $\mathrm{Lb}$ & - & - & 42 \\
\hline 589 & $\mathrm{RS}$ Cnc & 090737.8 & +311003 & 194.5 & +42.1 & M6IIIaSe & SRc? & 120 & $+7,+11$ & 23 \\
\hline 626 & FM Hya & 094110.3 & -182043 & 252.8 & +25.5 & MOS & Mira & 300 & - & 17 \\
\hline 704 & Z Ant & 104340.2 & -345917 & 275.9 & +20.9 & $\mathrm{~S} 5,4$ & $\mathrm{SR}$ & 104 & - & 10 \\
\hline 796 & HR 4755 & 122716.8 & -412735 & 298.6 & +21.0 & M2II - III & - & - & -2 & 10 \\
\hline 803 & S UMa & 124145.5 & +612200 & 124.6 & +56.0 & $\mathrm{~S} 3 / 6 \mathrm{e}$ & Mira & 226 & $+5,+18$ & 10 \\
\hline 816 & UY Cen & 131336.8 & -442627 & 307.6 & +17.9 & $\mathrm{~S} 6 / 8, \mathrm{CS}$ & $\mathrm{SR}$ & 114 & -23 & 39 \\
\hline
\end{tabular}

Notes to Table 4:

1. The spectral type in column 8 is from the GCGSS

2. Column 10 gives the observed range of optical velocities, taken from the following references: Brown et al. 1990 (GCGSS 149 and 589), Udry et al. (1998) and Jorissen et al. (1998) (GCGSS 117 and 212), Wilson 1953 (GCGSS 283, 803 and 816), Hoffleit (1982) (GCGSS 796)

3. All stars observed in the $\mathrm{CO}(2-1)$ line except GP Ori, NQ Pup and UY Cen, which were observed in the $\mathrm{CO}(3-2)$ line.

Table 5. $\mathrm{CO}(2-1)$ data for the four S stars detected by the CSO observations

\begin{tabular}{|c|c|c|c|c|c|}
\hline GCGSS & & $\begin{array}{c}I_{\mathrm{CO}} \\
\left(\mathrm{K} \times \mathrm{km} \mathrm{s}^{-1}\right) \\
\end{array}$ & $\begin{array}{c}T(\text { peak }) \\
(\mathrm{K})\end{array}$ & $\begin{array}{c}V_{\mathrm{c}} \\
\left(\mathrm{km} \mathrm{s}^{-1}\right)\end{array}$ & $\begin{array}{c}V_{\mathrm{e}} \\
\left(\mathrm{km} \mathrm{s}^{-1}\right) \\
\end{array}$ \\
\hline 212 & FU Mon & $0.63 \pm 0.05$ & $0.15 \pm 0.02$ & $-41.7 \pm 0.2$ & $2.8 \pm 0.3$ \\
\hline 283 & R Lyn & $0.89 \pm 0.19$ & $0.08 \pm 0.02$ & $+15.7 \pm 1.4$ & $9.3 \pm 1.7$ \\
\hline 589 & RS Cnc & $13.3 \pm 0.9$ & $\begin{array}{l}0.78 \\
117\end{array}$ & $\begin{array}{l}+7.7 \\
+6.8\end{array}$ & 7.4 \\
\hline 704 & Z Ant & $0.73 \pm 0.09$ & $\begin{array}{c}1.17 \\
0.07 \pm 0.02\end{array}$ & $\begin{array}{c}+6.8 \\
-15.6 \pm 1.0\end{array}$ & $\begin{array}{c}2.6 \\
7.4 \pm 1.3\end{array}$ \\
\hline
\end{tabular}




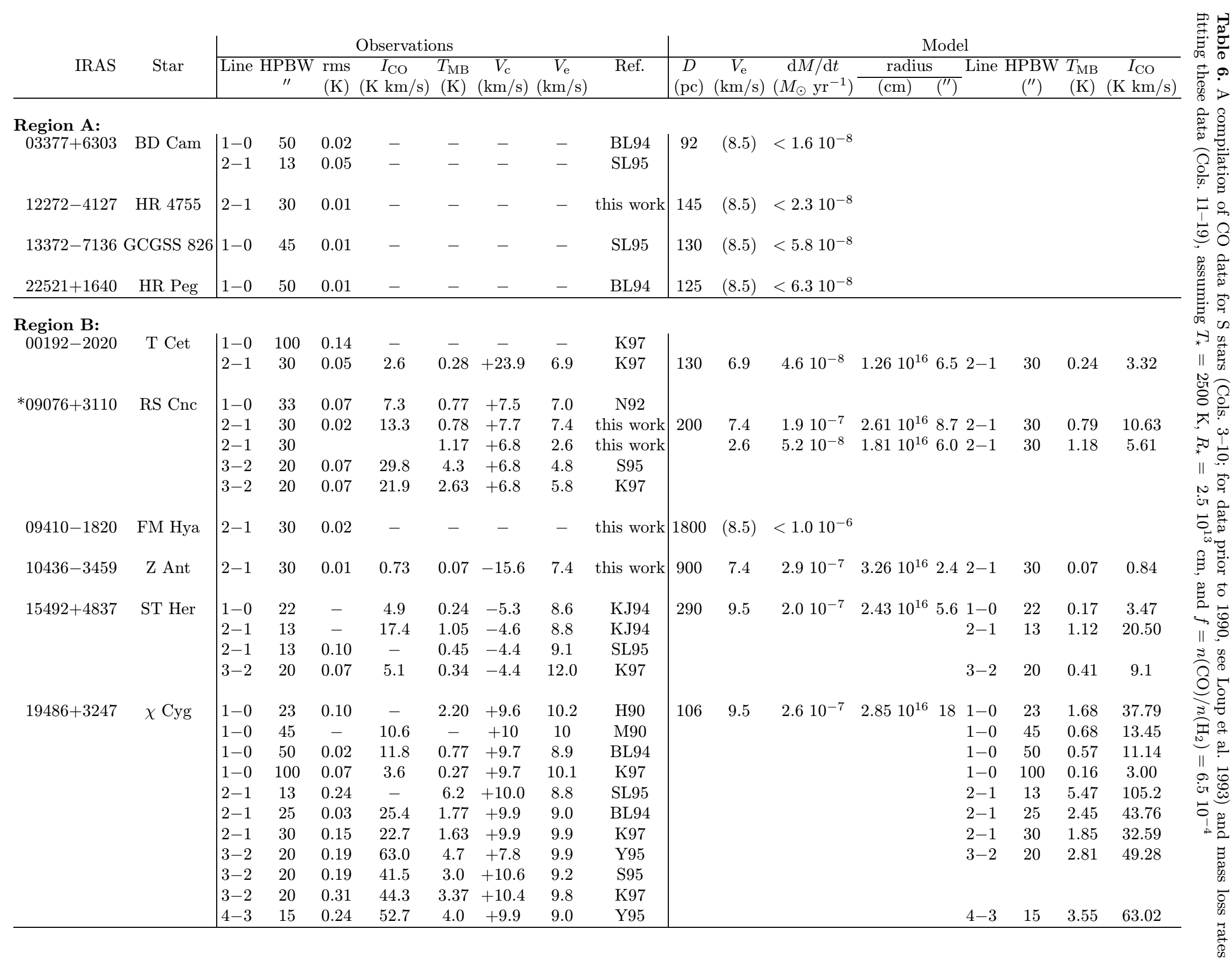




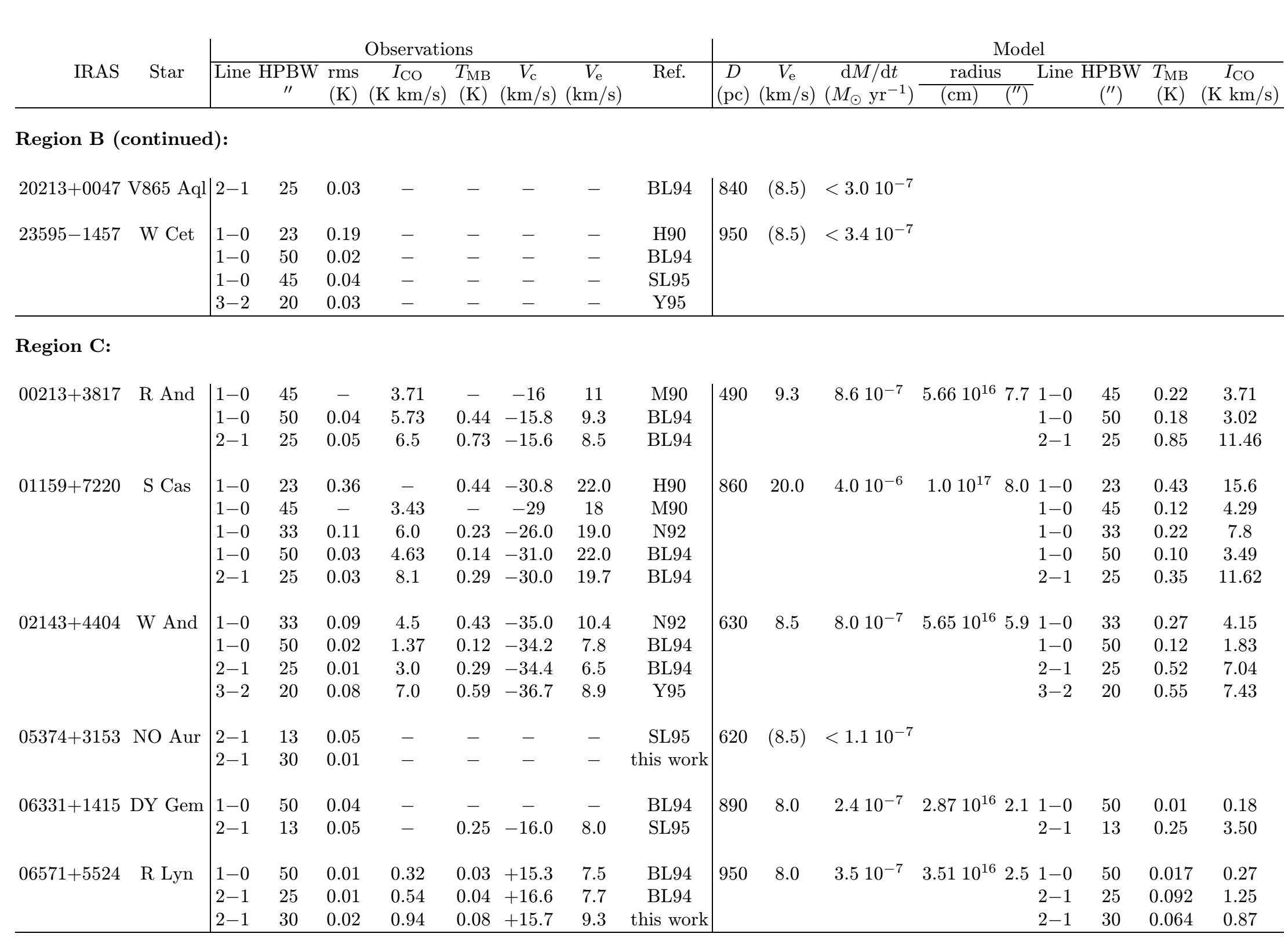




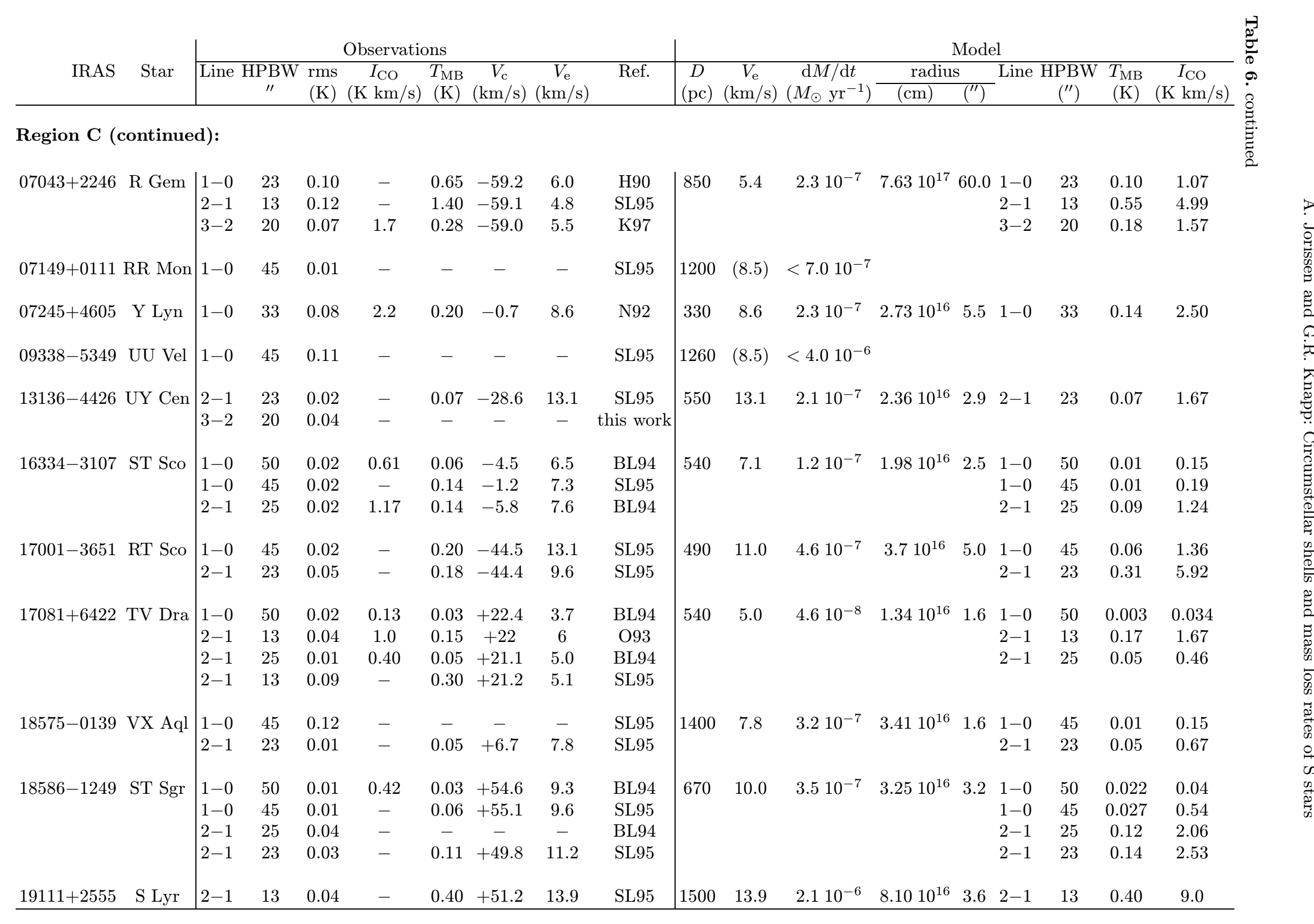




\begin{tabular}{|c|c|c|c|c|c|c|c|c|c|c|c|c|c|c|c|c|c|c|}
\hline \multirow[b]{2}{*}{ IRAS } & \multirow[b]{2}{*}{ Star } & \multicolumn{8}{|c|}{ Observations } & \multicolumn{9}{|c|}{ Model } \\
\hline & & Line & $\begin{array}{c}\text { HPBW } \\
\prime \prime\end{array}$ & $\begin{array}{l}\mathrm{rms} \\
(\mathrm{K}) \\
\end{array}$ & $\begin{array}{c}I_{\mathrm{CO}} \\
(\mathrm{K} \mathrm{km} / \mathrm{s})\end{array}$ & $\begin{array}{c}T_{\mathrm{MB}} \\
(\mathrm{K}) \\
\end{array}$ & $\begin{array}{c}V_{\mathrm{c}} \\
(\mathrm{km} / \mathrm{s}) \\
\end{array}$ & $\begin{array}{c}V_{\mathrm{e}} \\
(\mathrm{km} / \mathrm{s})\end{array}$ & Ref. & \begin{tabular}{|c|}
$D$ \\
$(\mathrm{pc})$ \\
\end{tabular} & $\begin{array}{c}V_{\mathrm{e}} \\
(\mathrm{km} / \mathrm{s})\end{array}$ & $\begin{array}{c}\mathrm{d} M / \mathrm{d} t \\
\left(M_{\odot} \mathrm{yr}^{-1}\right)\end{array}$ & \multicolumn{2}{|c|}{ radius } & \multicolumn{3}{|c|}{ Line HPBW $T_{\mathrm{MB}}$} & $\begin{array}{c}I_{\mathrm{CO}} \\
(\mathrm{K} \mathrm{km} / \mathrm{s})\end{array}$ \\
\hline \multicolumn{19}{|c|}{ Region C (continued): } \\
\hline $19126-0708$ & W Aql & $\begin{array}{l}1-0 \\
1-0 \\
1-0 \\
1-0 \\
1-0 \\
2-1 \\
2-1 \\
2-1 \\
2-1 \\
3-2\end{array}$ & $\begin{array}{l}23 \\
45 \\
45 \\
50 \\
45 \\
25 \\
30 \\
13 \\
23 \\
20\end{array}$ & $\begin{array}{c}0.09 \\
- \\
0.05 \\
0.03 \\
0.22 \\
0.04 \\
0.03 \\
0.16 \\
0.25 \\
0.10\end{array}$ & $\begin{array}{c}- \\
26.2 \\
28.4 \\
27.7 \\
- \\
43.1 \\
54.8 \\
- \\
- \\
93.2\end{array}$ & $\begin{array}{c}2.31 \\
- \\
0.96 \\
0.98 \\
1.30 \\
1.60 \\
2.10 \\
6.05 \\
2.04 \\
3.51\end{array}$ & $\begin{array}{c}-24.6 \\
-25 \\
-24.0 \\
-24.0 \\
-24.1 \\
-24.1 \\
-25.2 \\
-24.0 \\
-24.3 \\
-23.8\end{array}$ & $\begin{array}{c}19.3 \\
20 \\
18.1 \\
17.7 \\
18.3 \\
17.4 \\
19.3 \\
18.8 \\
18.0 \\
19.6\end{array}$ & $\begin{array}{c}\text { H90 } \\
\text { M90 } \\
\text { N92 } \\
\text { BL94 } \\
\text { SL95 } \\
\text { BL94 } \\
\text { K97 } \\
\text { SL95 } \\
\text { SL95 } \\
\text { K97 }\end{array}$ & 610 & 18.7 & $1.310^{-5}$ & $2.1210^{17}$ & 23.2 & $\begin{array}{l}1-0 \\
1-0 \\
1-0 \\
\\
2-1 \\
2-1 \\
2-1 \\
2-1 \\
3-2\end{array}$ & $\begin{array}{l}25 \\
30 \\
13 \\
23 \\
20\end{array}$ & $\begin{array}{l}2.98 \\
1.14 \\
0.95 \\
\\
26.7 \\
1.96 \\
6.98 \\
3.05 \\
2.70\end{array}$ & $\begin{array}{c}102.4 \\
\\
35.54 \\
29.5 \\
\\
78.2 \\
56.98 \\
217.3 \\
90.0 \\
76.95\end{array}$ \\
\hline $19133-1703$ & T Sgr & $\begin{array}{l}1-0 \\
1-0 \\
2-1 \\
2-1\end{array}$ & $\begin{array}{l}50 \\
45 \\
25 \\
23\end{array}$ & $\begin{array}{l}0.02 \\
0.02 \\
0.04 \\
0.02\end{array}$ & $\begin{array}{l}- \\
- \\
- \\
-\end{array}$ & $\begin{array}{c}- \\
- \\
- \\
0.06\end{array}$ & $\begin{array}{c}- \\
- \\
- \\
+10.8\end{array}$ & $\begin{array}{c}- \\
- \\
- \\
14.1\end{array}$ & $\begin{array}{l}\text { BL94 } \\
\text { SL95 } \\
\text { BL94 } \\
\text { SL95 }\end{array}$ & 810 & 14.1 & $4.010^{-7}$ & $3.2210^{16}$ & 2.7 & $2-1$ & 23 & 0.06 & 1.53 \\
\hline $19354+5005$ & R Cyg & $\begin{array}{l}1-0 \\
2-1 \\
3-2\end{array}$ & $\begin{array}{l}50 \\
25 \\
20\end{array}$ & $\begin{array}{l}0.01 \\
0.02 \\
0.17\end{array}$ & $\begin{array}{l}2.52 \\
5.04 \\
14.6\end{array}$ & $\begin{array}{c}0.15 \\
0.33 \\
1.3\end{array}$ & $\begin{array}{l}-17.5 \\
-17.2 \\
-18.2\end{array}$ & $\begin{array}{c}9.9 \\
11.4 \\
10.4\end{array}$ & $\begin{array}{c}\text { BL94 } \\
\text { BL94 } \\
\text { S95 }\end{array}$ & 900 & 10.5 & $1.710^{-6}$ & $8.1610^{16}$ & 6.1 & $\begin{array}{l}1-0 \\
2-1 \\
3-2\end{array}$ & $\begin{array}{l}50 \\
25 \\
20\end{array}$ & $\begin{array}{l}0.11 \\
0.43 \\
0.44\end{array}$ & $\begin{array}{c}2.0 \\
7.06 \\
7.18\end{array}$ \\
\hline $22036+3315$ & RZ Peg & $2-1$ & 13 & 0.04 & - & 0.21 & -23.4 & 12.6 & SL95 & 1560 & 12.6 & $1.110^{-6}$ & $5.9310^{16}$ & 2.5 & $2-1$ & 13 & 0.21 & 4.57 \\
\hline$* 22196-4612$ & $\pi^{1} \mathrm{Gru}$ & $\begin{array}{l}1-0 \\
1-0 \\
2-1 \\
2-1\end{array}$ & $\begin{array}{l}45 \\
45 \\
23 \\
23\end{array}$ & $\begin{array}{c}0.05 \\
0.08 \\
- \\
0.06\end{array}$ & $\begin{array}{l}6.9 \\
- \\
- \\
-\end{array}$ & $\begin{array}{l}0.33 \\
0.60 \\
2.20 \\
2.00\end{array}$ & $\begin{array}{l}-13.2 \\
-12.5\end{array}$ & $\begin{array}{l}19.4 \\
11.0\end{array}$ & $\begin{array}{c}\text { N92 } \\
\text { SL95 } \\
\text { S92 } \\
\text { SL95 }\end{array}$ & 160 & 11.0 & $4.610^{-7}$ & $3.7010^{16}$ & 15.5 & $1-0$ & 45 & $\begin{array}{l}0.53 \\
2.20\end{array}$ & $\begin{array}{l}11.80 \\
45.67\end{array}$ \\
\hline $23554+5612$ & WY Cas & $2-1$ & 13 & 0.04 & - & 0.50 & +7.5 & 13.3 & SL95 & 1300 & 13.3 & $1.810^{-6}$ & $7.6510^{16}$ & 3.9 & $2-1$ & 13 & 0.50 & 1.08 \\
\hline
\end{tabular}




\begin{tabular}{|c|c|c|c|c|c|c|c|c|c|c|c|c|c|c|c|c|c|c|}
\hline \multirow[b]{2}{*}{ IRAS } & \multirow[b]{2}{*}{ Star } & \multicolumn{8}{|c|}{ Observations } & \multicolumn{9}{|c|}{ Model } \\
\hline & & Line & $\begin{array}{l}\text { HPBW } \\
=\end{array}$ & $\begin{array}{l}\mathrm{rms} \\
(\mathrm{K}) \\
\end{array}$ & $\begin{array}{c}I_{\mathrm{CO}} \\
(\mathrm{K} \mathrm{km} / \mathrm{s}) \\
\end{array}$ & $\begin{array}{l}T_{\mathrm{MB}} \\
(\mathrm{K}) \\
\end{array}$ & $\begin{array}{c}V_{\mathrm{c}} \\
(\mathrm{km} / \mathrm{s}) \\
\end{array}$ & $\begin{array}{c}V_{\mathrm{e}} \\
(\mathrm{km} / \mathrm{s})\end{array}$ & Ref. & $\begin{array}{c}D \\
(\mathrm{pc})\end{array}$ & $\begin{array}{c}V_{\mathrm{e}} \\
(\mathrm{km} / \mathrm{s})\end{array}$ & $\begin{array}{c}\mathrm{d} M / \mathrm{d} t \\
\left(M_{\odot} \mathrm{yr}^{-1}\right)\end{array}$ & $\begin{array}{l}\text { radius } \\
(\mathrm{cm}) \\
\end{array}$ & $\left({ }^{\prime \prime}\right)$ & Line & $\begin{array}{c}\text { HPBW } \\
\left({ }^{\prime \prime}\right)\end{array}$ & $\begin{array}{l}T_{\mathrm{MB}} \\
(\mathrm{K}) \\
\end{array}$ & $\begin{array}{c}I_{\mathrm{CO}} \\
(\mathrm{K} \mathrm{km} / \mathrm{s}) \\
\end{array}$ \\
\hline \multicolumn{19}{|l|}{ Region D: } \\
\hline $00578+5620$ & V365 Cas & $\mid \begin{array}{l}1-0 \\
2-1 \\
2-1\end{array}$ & $\begin{array}{l}50 \\
25 \\
13\end{array}$ & $\begin{array}{l}0.01 \\
0.03 \\
0.02\end{array}$ & $\begin{array}{l}- \\
- \\
-\end{array}$ & $\begin{array}{c}- \\
- \\
0.07\end{array}$ & $\begin{array}{c}- \\
- \\
-2.1\end{array}$ & $\begin{array}{l}- \\
- \\
7.2\end{array}$ & $\begin{array}{l}\text { BL94 } \\
\text { BL94 } \\
\text { SL95 }\end{array}$ & 800 & 7.2 & $7.010^{-8}$ & $1.5310^{16}$ & 1.3 & $2-1$ & 13 & 0.07 & 0.98 \\
\hline $04352+6602$ & T Cam & $\begin{array}{l}1-0 \\
2-1\end{array}$ & $\begin{array}{l}50 \\
25\end{array}$ & $\begin{array}{l}0.02 \\
0.05\end{array}$ & $\begin{array}{l}- \\
-\end{array}$ & $\begin{array}{l}- \\
-\end{array}$ & $\begin{array}{l}- \\
-\end{array}$ & $\begin{array}{l}- \\
-\end{array}$ & $\begin{array}{l}\text { BL94 } \\
\text { BL94 }\end{array}$ & 520 & $(8.5)$ & $<2.210^{-7}$ & & & & & & \\
\hline $04543+4829$ & TV Aur & $\begin{array}{l}1-0 \\
2-1\end{array}$ & $\begin{array}{l}50 \\
25\end{array}$ & $\begin{array}{l}0.02 \\
0.03\end{array}$ & $\begin{array}{l}- \\
-\end{array}$ & $\begin{array}{l}- \\
-\end{array}$ & $\begin{array}{l}- \\
-\end{array}$ & $\begin{array}{l}- \\
-\end{array}$ & $\begin{array}{l}\text { BL94 } \\
\text { BL94 }\end{array}$ & 780 & $(8.5)$ & $<2.610^{-7}$ & & & & & & \\
\hline $07095+6853$ & AA Cam & $2-1$ & 13 & 0.02 & - & 0.06 & -43.5 & 17.9 & SL95 & 880 & 17.9 & $2.910^{-7}$ & $2.6710^{16}$ & 2.0 & $2-1$ & 13 & 0.06 & 2.14 \\
\hline $07399-1045$ & SU Mon & $\begin{array}{l}1-0 \\
1-0 \\
2-1\end{array}$ & $\begin{array}{l}50 \\
45 \\
25\end{array}$ & $\begin{array}{l}0.01 \\
0.01 \\
0.03\end{array}$ & $\begin{array}{l}- \\
- \\
-\end{array}$ & $\begin{array}{l}- \\
- \\
-\end{array}$ & $\begin{array}{l}- \\
- \\
-\end{array}$ & $\begin{array}{l}- \\
- \\
-\end{array}$ & $\begin{array}{l}\text { BL94 } \\
\text { SL95 } \\
\text { BL94 }\end{array}$ & 780 & $(8.5)$ & $<2.610^{-7}$ & & & & & & \\
\hline $12417+6121$ & S UMa & $2-1$ & 30 & 0.01 & - & - & - & - & this work & 1650 & $(8.5)$ & $<4.810^{-7}$ & & & & & & \\
\hline$* 13440-5306$ & AM Cen & $\begin{array}{l}1-0 \\
2-1\end{array}$ & $\begin{array}{l}45 \\
23\end{array}$ & $\begin{array}{l}0.02 \\
0.04\end{array}$ & $\begin{array}{l}- \\
-\end{array}$ & $\begin{array}{l}0.08 \\
0.20\end{array}$ & -27.4 & 5.4 & $\begin{array}{l}\text { SL95 } \\
\text { SL95 }\end{array}$ & 910 & 5.4 & $3.510^{-7}$ & $4.1310^{16}$ & 3.0 & $\begin{array}{l}1-0 \\
2-1\end{array}$ & $\begin{array}{l}45 \\
23\end{array}$ & $\begin{array}{c}0.054 \\
0.27\end{array}$ & $\begin{array}{l}0.55 \\
2.33\end{array}$ \\
\hline $13477-6009$ & VX Cen & $1-0$ & 45 & 0.03 & - & - & - & - & SL95 & 530 & $(8.5)$ & $<4.610^{-7}$ & & & & & & \\
\hline $17206-2826$ & V521 Oph & $\begin{array}{l}1-0 \\
1-0\end{array}$ & $\begin{array}{l}50 \\
45\end{array}$ & $\begin{array}{l}0.01 \\
0.01\end{array}$ & - & - & - & - & $\begin{array}{l}\text { BL94 } \\
\text { SL95 }\end{array}$ & 580 & $(8.5)$ & $<2.510^{-7}$ & & & & & & \\
\hline $19311+2332$ & EP Vul & $\begin{array}{l}1-0 \\
1-0 \\
2-1 \\
2-1\end{array}$ & $\begin{array}{l}50 \\
45 \\
25 \\
13\end{array}$ & $\begin{array}{l}0.02 \\
0.01 \\
0.02 \\
0.14\end{array}$ & $\begin{array}{c}0.94 \\
- \\
1.56 \\
-\end{array}$ & $\begin{array}{l}0.12 \\
0.08 \\
0.20 \\
0.52\end{array}$ & $\begin{array}{l}-0.2 \\
-0.7 \\
+0.1 \\
-0.1\end{array}$ & $\begin{array}{l}5.2 \\
5.6 \\
5.5 \\
4.7\end{array}$ & $\begin{array}{l}\text { BL94 } \\
\text { SL95 } \\
\text { BL94 } \\
\text { SL95 }\end{array}$ & 650 & 5.4 & $2.910^{-7}$ & $3.7010^{16}$ & 3.8 & $\begin{array}{l}1-0 \\
1-0 \\
2-1\end{array}$ & $\begin{array}{l}50 \\
45 \\
25\end{array}$ & $\begin{array}{l}0.07 \\
0.08 \\
0.34\end{array}$ & $\begin{array}{l}0.68 \\
0.84 \\
2.99\end{array}$ \\
\hline $20026+3640$ & AA Cyg & $\begin{array}{l}1-0 \\
2-1\end{array}$ & $\begin{array}{l}50 \\
13\end{array}$ & $\begin{array}{l}0.03 \\
0.13\end{array}$ & $\begin{array}{c}1.51 \\
-\end{array}$ & $\begin{array}{l}0.11 \\
0.95\end{array}$ & $\begin{array}{l}+22.7 \\
+27.9\end{array}$ & $\begin{array}{l}9.4 \\
4.8\end{array}$ & $\begin{array}{l}\text { BL94 } \\
\text { SL95 }\end{array}$ & 550 & 7.1 & $3.110^{-7}$ & $3.4610^{16}$ & 4.2 & $\begin{array}{l}1-0 \\
2-1\end{array}$ & $\begin{array}{l}50 \\
13\end{array}$ & $\begin{array}{l}0.06 \\
1.05\end{array}$ & $\begin{array}{c}0.80 \\
12.82\end{array}$ \\
\hline $22476+4047$ & RX Lac & $\begin{array}{l}1-0 \\
2-1\end{array}$ & $\begin{array}{l}45 \\
13\end{array}$ & $\begin{array}{l}0.13 \\
0.12\end{array}$ & $\begin{array}{l}- \\
-\end{array}$ & $\begin{array}{c}- \\
0.68\end{array}$ & $\begin{array}{c}- \\
-15.4\end{array}$ & $\begin{array}{c}- \\
3.4\end{array}$ & $\begin{array}{l}\text { SL95 } \\
\text { SL95 }\end{array}$ & 380 & 3.4 & $4.010^{-8}$ & $1.410^{16}$ & 4.1 & $2-1$ & 13 & 0.67 & 4.42 \\
\hline
\end{tabular}




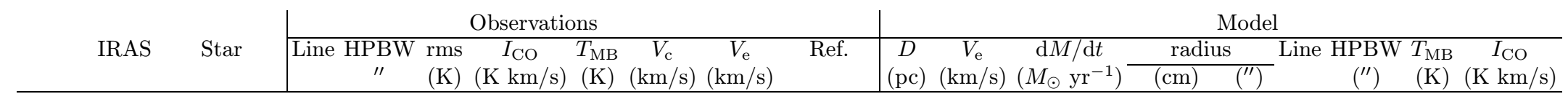

\section{Region E:}

\begin{tabular}{|c|c|c|c|c|c|c|c|c|c|c|c|c|c|c|c|c|c|c|}
\hline $04123+2357$ & GCGSS89 & $2-1$ & 30 & 0.02 & - & - & - & - & this work & 650 & $(8.5)$ & $<1.710^{-7}$ & & & & & & \\
\hline $04497+1410$ & $o^{1}$ Ori & $1-0$ & 45 & 0.02 & - & - & - & - & SL95 & 330 & $(8.5)$ & $<2.010^{-7}$ & & & & & & \\
\hline $04599+1514$ & GP Ori & $3-2$ & 20 & 0.04 & - & - & - & - & this work & 1100 & $(8.5)$ & $<5.210^{-7}$ & & & & & & \\
\hline$* 06197+0327$ & FU Mon & $2-1$ & 30 & 0.01 & 0.66 & 0.16 & -41.7 & 2.8 & this work & 860 & 2.8 & $1.010^{-7}$ & $7.7010^{17}$ & 59.8 & $2-1$ & 30 & 0.14 & 0.63 \\
\hline $07507-1129$ & NQ Pup & $3-2$ & 20 & 0.04 & - & - & - & - & this work & 1160 & $(8.5)$ & $<6.310^{-7}$ & & & & & & \\
\hline $13163-6031$ & TT Cen & $\begin{array}{l}1-0 \\
2-1\end{array}$ & $\begin{array}{l}45 \\
23\end{array}$ & $\begin{array}{l}0.04 \\
0.05\end{array}$ & - & $\begin{array}{l}0.05 \\
0.24\end{array}$ & $\begin{array}{l}+5.2 \\
+5.5\end{array}$ & $\begin{array}{l}24.0 \\
25.4\end{array}$ & $\begin{array}{l}\text { SL95 } \\
\text { SL95 }\end{array}$ & 1070 & 24.7 & $4.610^{-6}$ & $1.010^{17}$ & 6.2 & $\begin{array}{l}1-0 \\
2-1\end{array}$ & $\begin{array}{l}45 \\
23\end{array}$ & $\begin{array}{l}0.06 \\
0.21\end{array}$ & $\begin{array}{l}2.67 \\
8.92\end{array}$ \\
\hline $20044+2417$ & DK Vul & $\begin{array}{l}1-0 \\
2-1 \\
2-1\end{array}$ & $\begin{array}{l}50 \\
25 \\
13\end{array}$ & $\begin{array}{l}0.02 \\
0.02 \\
0.05\end{array}$ & $\begin{array}{c}1.33 \\
1.41 \\
-\end{array}$ & $\begin{array}{l}0.04 \\
0.21 \\
1.10\end{array}$ & $\begin{array}{l}-13.9 \\
-14.2 \\
-14.2\end{array}$ & $\begin{array}{l}8.9 \\
5.3 \\
4.7\end{array}$ & $\begin{array}{l}\text { BL94 } \\
\text { BL94 } \\
\text { SL95 }\end{array}$ & 910 & 5.0 & $2.910^{-7}$ & $3.8310^{16}$ & 2.8 & $\begin{array}{l}1-0 \\
2-1 \\
2-1\end{array}$ & $\begin{array}{l}50 \\
25 \\
13\end{array}$ & $\begin{array}{l}0.04 \\
0.20 \\
0.74\end{array}$ & $\begin{array}{l}0.38 \\
1.64 \\
5.93\end{array}$ \\
\hline $20100-6225$ & GCGSS1195 & $\begin{array}{l}1-0 \\
2-1\end{array}$ & $\begin{array}{l}45 \\
23\end{array}$ & $\begin{array}{l}0.04 \\
0.14\end{array}$ & $\begin{array}{l}- \\
-\end{array}$ & $\begin{array}{l}- \\
-\end{array}$ & $\begin{array}{l}- \\
-\end{array}$ & $\begin{array}{l}- \\
-\end{array}$ & $\begin{array}{l}\text { SL95 } \\
\text { SL95 }\end{array}$ & 900 & $(8.5)$ & $<1.010^{-6}$ & & & & & & \\
\hline $20120-4433$ & RZ Sgr & $\begin{array}{l}1-0 \\
2-1\end{array}$ & $\begin{array}{l}45 \\
23\end{array}$ & $\begin{array}{l}0.03 \\
0.04\end{array}$ & $\begin{array}{l}- \\
-\end{array}$ & $\begin{array}{l}0.36 \\
0.98\end{array}$ & $\begin{array}{l}-31.6 \\
-31.2\end{array}$ & $\begin{array}{l}14 \\
8.8\end{array}$ & $\begin{array}{l}\text { SL95 } \\
\text { SL95 }\end{array}$ & 820 & 8.8 & $2.110^{-6}$ & $9.9010^{16}$ & 8.1 & $\begin{array}{l}1-0 \\
2-1\end{array}$ & $\begin{array}{l}45 \\
23\end{array}$ & $\begin{array}{l}0.30 \\
1.08\end{array}$ & $\begin{array}{l}4.36 \\
14.6\end{array}$ \\
\hline
\end{tabular}

References:

H90: Heske 1990; M90: Margulis et al. 1990; S92: Sahai 1992; N92: Nyman et al. 1992; N93: Nyman et al. 1993; O93: Omont et al. 1993; KJ94: Kahane \& Jura 1994; BL94: Bieging \& Latter 1994; SL95: Sahai \& Liechti 1995; Y95: Young 1995; S95: Stanek et al. 1995; K97: Knapp et al. 1997b.

Notes:

1. $06197+0327$, FU Mon: Data from Sahai \& Liechti (1995) not used - see text;

2. $09076+3110$, RS Cnc: $\mathrm{CO}(2-1)$ line profile has two components - see text;

3. $13440-5306$, AM Cen: Values for central velocity $V_{\mathrm{c}}$ and outflow velocity $V_{\mathrm{e}}$ given by SL95 are averages of the values for the $\mathrm{CO}(1-0)$ and $\mathrm{CO}(2-1)$ lines;

4. $22196-4612, \pi^{1}$ Gru: SL95 do not list values for $V_{\mathrm{c}}$ and $V_{\mathrm{e}}$ because of the line wings. 